\title{
Diffraction coefficients of a semi-infinite planar crack embedded in a transversely-isotropic space
}

\author{
A. Gautesen ${ }^{\mathrm{a}}$, V. Zernov ${ }^{\mathrm{b}, *}$, and L. Fradkin ${ }^{\mathrm{b}}$ \\ ${ }^{a}$ Dept. of Mathematics, Iowa State University and Ames Laboratory, Ames, IA 50011, U.S.A. \\ ${ }^{\mathrm{b}}$ Waves and Fields Research Group, Department of Electrical, Computer and Communication \\ Engineering, Faculty of Engineering, Science and Built Environment, London South Bank University, \\ SE1 OAA, U.K.
}

\begin{abstract}
We have considered a semi-infinite crack embedded in a transversely isotropic medium and studied two special cases, one, in which the axis of symmetry is normal to the crack face and the wave incidence is arbitrary and another, in which the axis lies in the crack plane normal to the edge and the incident wave vector is also normal to the edge. The problem is of interest in Non-Destructive Evaluation, because austenitic steels that are found in claddings and other welds in the nuclear reactors are often modeled as transversely isotropic. In both of cases, we have expressed the scattered field in a closed form and computed the corresponding diffraction coefficients.
\end{abstract}

Key words: diffraction coefficients, transversely isotropic medium, elastic waves PACS: 43.20.El, 43.20.Gp

\section{Introduction}

The main aim of this article is to evaluate - in two special cases - diffraction coefficients of a semi-infinite planar crack embedded into a TI (transversely isotropic) solid. TI is an anisotropic medium which is invariant under the rotation around a symmetry axis, that is, a TI medium exhibits isotropy in the plane perpendicular to that axis. The symmetry of this kind is ubiquitous in nature and manmade materials and TI solids have been widely studied e.g. in crystallography, seismology and NDE (Non-Destructive Evaluation).

\footnotetext{
* Corresponding author.

Email address: zernovv@lsbu.ac.uk (V. Zernov).
} 
The diffraction coefficients relate the far field amplitudes of the corresponding waves diffracted by a straight crack edge to the amplitude of an incident plane wave. The rays, along which energy propagates and the wave fronts, which separate the disturbed regions in space from undisturbed, are the basic concepts of the so-called ray theory. In the far field (high-frequency) approximation, this provides a convenient description of the wave phenomena such as propagation, reflection and refraction (see e.g. [2]).

It is well known that in an isotropic solid, the fronts of waves radiated by a point source comprise three concentric spheres. At each moment in time, the sphere of the largest radius is the region covered by the disturbance due to $\mathrm{P}$ (compressional or longitudinal) mode of propagation while the remaining two spheres are coincident and describe the region disturbed by $\mathrm{S}$ (shear or transverse) waves. The latter degeneracy is usually resolved by identifying two orthogonal components of polarization, which are named the SV and $\mathrm{SH}$ modes, with $\mathrm{V}$ standing for vertical and $\mathrm{H}$ for horizontal. The rays are normal to wave fronts. The nature of wave propagation in anisotropic elastic solids is significantly different, since they support three distinct types of elastic waves, qP, qSV and qSH, with q standing for quasi, none of which is in general purely compressional or shear. The rays are not normal to the wave fronts either [3]

A well known extension of the ray theory, GTD (the Geometrical Theory of Diffraction), suitable for description of diffraction in the far-field approximation was first introduced by Keller [4]-[6]. He considered diffraction of the plane scalar wave by an edge embedded into an isotropic medium whose radius of curvature is much larger than the wave length. According to GTD, a plane wave incident on such an edge produces a cone of diffracted rays. The apex of the cone coincides with the point of diffraction; it is centered on the straight line which is tangent to the edge at this point, and the cone's solid angle is determined by the Snell's law. Moreover, according to Keller, GTD applies even when the incident wave is not plane, but at the point where it strikes the edge, the radii of curvature of the wave front are much larger than the wave length. Special cases which can be treated using the high-frequency approximation but for which GTD fails can be described with the so-called uniform GTD which lies outside the scope of this article.

In [1] Keller's GTD was extended to elastodynamics and derived formulae for the diffraction coefficients for a semi-infinite planar crack in an isotropic solid. They achieved this by reformulating the original problem as a Wiener-Hopf matrix functional equation in a complex Fourier variable. The problem was solved by the Wiener-Hopf factorization technique (see e.g. [15]) and explicit formulae for the diffraction coefficients were derived using the method of steepest descent. The latter provides an explicit construction of the diffraction cones as well as complex amplitudes along the rays.

For a general anisotropic solid, a semi-analytical approach to the problem was previously developed in [13]. The authors have reduced the problem to a Wiener-Hopf functional equation which, in general, has no known analytical solution and have used a numerical scheme to factorize the underlying Wiener-Hopf matrix. In [14], they gave a description of numerics and showed the dependence of the magnitude of the backscatter $q P-q P$ diffraction coefficient on the observation (incidence) angle.

Here we aim to produce a procedure for calculating some diffraction coefficients for semi-infinite planar cracks in austenitic steels. The problem is of interest in NDE, because austenitic steels are found in claddings and other welds in the nuclear reactors. It is well known that the austenitic steel can be modeled as a TI material (see e.g. [12]). A simple case of normal incidence in a TI material that supports three convex slowness surfaces (see 
below) has been considered before [7]. We address a more challenging oblique incidence case and deal with an extra complication due to the fact that in the austenitic steel, one of the slowness surfaces has inflections.

The article is organized as follows: in Section 2 we state the problem in terms of partial differential equations and boundary conditions. In Section 3 we discuss the corresponding transfer tensor, that is the free-space Green's tensor in the Fourier domain. In Section 4 we reformulate the problem in terms of an integral equation (Green's formula). In Section 5 we reformulate it again as a $3 \mathrm{D}$ functional equation and solve this equation analytically in Section 6 for the case of the symmetry axis perpendicular to the crack plane and in Section 7 for the case of the symmetry axis lying in the crack plane perpendicular to the crack edge. In Section 8 we calculate the corresponding diffraction coefficients.

\section{The problem statement}

Let the medium be a homogeneous elastic solid governed by the Hooke's law

$$
\sigma_{i j}^{d}\left(\mathbf{x}^{d}, t\right)=c_{i j k l}^{d} \epsilon_{k l}\left(\mathbf{x}^{d}, t\right),
$$

where the superscript ${ }^{d}$ is used to denote the dimensional quantities (whenever the nondimensional versions are also used), $\mathbf{x}^{d}$ is an arbitrary point in space, $t$ is time and everywhere $\sigma^{d}\left(\mathbf{x}^{d}, t\right)$ and $\epsilon\left(\mathbf{x}^{d}, t\right)$ are respectively, stress and strain tensor of the second order while $i, j, k, l=1,2,3$ are indices of the tensor component corresponding to any three dimensional Cartesian coordinate system. Here and everywhere below, unless otherwise stated, we employ the summation convention over the repeated index.

As already mentioned above, a TI material has one axis of symmetry. It is well known that the corresponding stiffness tensor $c^{d}$ involves five unknowns [3]. Also, the stress tensor $\sigma^{d}\left(\mathbf{x}^{d}, t\right)$ and strain tensor $\epsilon\left(\mathbf{x}^{d}, t\right)$ are both symmetric, which allows us to reduce their order by using the so-called Voigt notations: Introducing the medium Cartesian coordinate system $\left\{\mathbf{e}_{1}, \mathbf{e}_{2}, \mathbf{e}_{3}\right\}$, with the $\mathbf{e}_{3}$ axis running along the symmetry axis, we replace the tensor $\sigma^{d}\left(\mathbf{x}^{d}, t\right)$ by the six dimensional vector whose the components $\sigma_{11}^{d}\left(\mathbf{x}^{d}, t\right), \sigma_{22}^{d}\left(\mathbf{x}^{d}, t\right), \sigma_{33}^{d}\left(\mathbf{x}^{d}, t\right)$ and $\sigma_{23}^{d}\left(\mathbf{x}^{d}, t\right), \sigma_{31}^{d}\left(\mathbf{x}^{d}, t\right), \sigma_{12}^{d}\left(\mathbf{x}^{d}, t\right)$ are called the normal and shear stresses, respectively. Then the Hooke's law (1) takes the form

$$
\left(\begin{array}{c}
\sigma_{11}^{d}\left(\mathbf{x}^{d}, t\right) \\
\sigma_{22}^{d}\left(\mathbf{x}^{d}, t\right) \\
\sigma_{33}^{d}\left(\mathbf{x}^{d}, t\right) \\
\sigma_{23}^{d}\left(\mathbf{x}^{d}, t\right) \\
\sigma_{31}^{d}\left(\mathbf{x}^{d}, t\right) \\
\sigma_{12}^{d}\left(\mathbf{x}^{d}, t\right)
\end{array}\right)=\left(\begin{array}{cccccc}
A_{11}^{d} & A_{12}^{d} & A_{13}^{d} & 0 & 0 & 0 \\
A_{12}^{d} & A_{11}^{d} & A_{13}^{d} & 0 & 0 & 0 \\
A_{13}^{d} & A_{13}^{d} & A_{33}^{d} & 0 & 0 & 0 \\
0 & 0 & 0 & 2 B_{1}^{d} & 0 & 0 \\
0 & 0 & 0 & 0 & 2 B_{1}^{d} & 0 \\
0 & 0 & 0 & 0 & 0 & 2 B_{3}^{d}
\end{array}\right) \cdot\left(\begin{array}{c}
\epsilon_{11}\left(\mathbf{x}^{d}, t\right) \\
\epsilon_{22}\left(\mathbf{x}^{d}, t\right) \\
\epsilon_{33}\left(\mathbf{x}^{d}, t\right) \\
\epsilon_{23}\left(\mathbf{x}^{d}, t\right) \\
\epsilon_{31}\left(\mathbf{x}^{d}, t\right) \\
\epsilon_{12}\left(\mathbf{x}^{d}, t\right)
\end{array}\right),
$$

where $B_{3}^{d}=\left(A_{11}^{d}-A_{12}^{d}\right) / 2$. It follows that any TI medium is characterized by five independent elastic moduli $A_{11}^{d}, A_{12}^{d}, A_{13}^{d}, A_{33}^{d}$ and $B_{1}^{d}$ as well as density $\rho$.

To continue, in the absence of body forces, the elastodynamic equation can be written as 


$$
\nabla^{d} \cdot \sigma^{d}(\mathbf{x}, t)=\rho \partial_{t}^{2} \mathbf{u}^{d}(\mathbf{x}, t)
$$

where the nabla operator $\nabla^{d}=\left(\partial_{1}^{d}, \partial_{2}^{d}, \partial_{3}^{d}\right)$, $\partial_{t}$ is the partial derivative with the respect to time and $\mathbf{u}^{d}(\mathbf{x}, t)$ is the displacement. Let us further assume that the medium is irradiated with an incident wave $\mathbf{u}^{d(i n)}(\mathbf{x}, t)$, which is harmonic, plane and has amplitude $U_{0}^{d}$. The corresponding stress, strain and displacement fields can be written as $\sigma^{d(s c)}(\mathbf{x}, t)=\sigma_{m}^{d(s c)}(\mathbf{x}) \exp (-\mathrm{i} \omega t), \epsilon^{(s c)}(\mathbf{x}, t)=\epsilon_{m}^{(s c)}(\mathbf{x}) \exp (-\mathrm{i} \omega t)$ and $\mathbf{u}^{d(s c)}(\mathbf{x}, t)=$ $\mathbf{u}_{m}^{d(s c)}(\mathbf{x}) \exp (-\mathrm{i} \omega t)$, respectively, where the subscript ${ }_{m}$ denotes functions whose vector arguments are expressed in the medium coordinates. Below we simplify the presentation by dropping the factor $\exp (-\mathrm{i} \omega t)$ everywhere. A further simplification can be achieved by non-dimesionalizing all physical variables, except $\omega$ and $t$ which do not feature below, using the material density $\rho$, the $\mathrm{S}$ wave speed along the symmetry axis $c_{0}$ (where a degeneracy takes place and there is no distinction between the $q S V$ and $q S H$ modes), reference wave number $k_{0}=\omega / c_{0}$ and the amplitude of the incident wave $U_{0}^{d}$, that is by introducing non-dimensional variables

$$
\begin{aligned}
& A_{i j}=\frac{A_{i j}^{d}}{\rho c_{0}^{2}}, \quad B_{i}=\frac{B_{i}^{d}}{\rho c_{0}^{2}}, \quad \sigma=\frac{\sigma^{d}}{\rho c_{0}^{2}} \\
& \mathbf{k}=k_{0}^{-1} \mathbf{k}^{d}, \quad \mathbf{u}=\left(U_{0}^{d}\right)^{-1} \mathbf{u}^{d}, \\
& \mathbf{x}=k_{0} \mathbf{x}^{d}, \quad \nabla=k_{0}^{-1} \nabla^{d} .
\end{aligned}
$$

The dimensionless form of the reduced elastodynamic equation is

$$
\nabla \cdot \sigma+\mathbf{u}=0
$$

where $\sigma=\sigma_{m}(\mathbf{x})$ and $\mathbf{u}=\mathbf{u}_{m}(\mathbf{x})$. Since for small deformations, the components of strain tensor $\epsilon=\epsilon_{m}(\mathbf{x})$ are defined by

$$
\epsilon_{i j}=\frac{1}{2}\left(\partial_{j} u_{i}+\partial_{i} u_{j}\right)
$$

the dimensionless form of the Hooke's law (1) allows us to relate the stress tensor to the displacement using the formula

$$
\sigma_{i j}=\Sigma_{j k}^{(i)}(\nabla) u_{k}
$$

where $\Sigma(\nabla)$ is a differential operator with the elements $\Sigma^{(1)}, \Sigma^{(2)}$ and $\Sigma^{(3)}$, which are the $3 \times 3$ matrices 


$$
\begin{gathered}
\Sigma^{(1)}(\nabla)=\left[\begin{array}{ccc}
A_{11} \partial_{1} & A_{12} \partial_{2} & A_{13} \partial_{3} \\
B_{3} \partial_{2} & B_{3} \partial_{1} & 0 \\
B_{1} \partial_{3} & 0 & B_{1} \partial_{1}
\end{array}\right], \\
\Sigma^{(2)}(\nabla)=\left[\begin{array}{ccc}
B_{3} \partial_{2} & B_{3} \partial_{1} & 0 \\
A_{12} \partial_{1} & A_{11} \partial_{2} & A_{13} \partial_{3} \\
0 & B_{1} \partial_{3} & B_{1} \partial_{2},
\end{array}\right], \\
\Sigma^{(3)}(\nabla)=\left[\begin{array}{ccc}
B_{1} \partial_{3} & 0 & B_{1} \partial_{1} \\
0 & B_{1} \partial_{3} & B_{1} \partial_{2} \\
A_{13} \partial_{1} & A_{13} \partial_{2} & A_{33} \partial_{3}
\end{array}\right] .
\end{gathered}
$$

Note that the stress tensor is symmetric, $\sigma_{i j}=\sigma_{j i}$, and therefore, the divergence of the stress tensor can be written as

$$
\nabla \cdot \sigma=L(\nabla) \mathbf{u},
$$

where in the medium coordinate system the operator $L=L(\nabla)$ is given by

$$
\begin{aligned}
& L_{11}(\nabla)=A_{11} \partial_{1}^{2}+B_{3} \partial_{2}^{2}+B_{1} \partial_{3}^{2}, \\
& L_{22}(\nabla)=B_{3} \partial_{1}^{2}+A_{11} \partial_{2}^{2}+B_{1} \partial_{3}^{2}, \\
& L_{33}(\nabla)=B_{1} \partial_{1}^{2}+B_{1} \partial_{2}^{2}+A_{33} \partial_{3}^{2}, \\
& L_{12}(\nabla)=L_{21}(\nabla)=\left(A_{11}-B_{3}\right) \partial_{1} \partial_{2}, \\
& L_{j 3}(\nabla)=L_{3 j}(\nabla)=\left(A_{13}+B_{1}\right) \partial_{j} \partial_{3}, \quad j=1,2 .
\end{aligned}
$$

It follows that elastodynamic equation (5) can be rewritten as

$$
L \mathbf{u}+\mathbf{u}=\mathbf{0} .
$$

Let us now assume that the medium contains a semi-infinite planar crack with noncontacting faces (see Fig. 1). Then the problem (11) should be supplemented with the boundary conditions: Firstly, we assume both crack faces $\Gamma^{+}$and $\Gamma^{-}$to be traction-free (see Fig. 1), so that we have

$$
\left.\mathbf{t}\right|_{\Gamma^{ \pm}}=\mathbf{0},
$$

where the traction $\mathbf{t}=\mathbf{t}(\mathbf{x})$ is a vector defined by

$$
\mathbf{t}=\boldsymbol{\nu} \cdot \sigma
$$

with $\boldsymbol{\nu}$ - the inner normal to the upper crack face (pointing into the solid). Note that using (7), we can write

$$
\mathbf{t}=S_{m}(\nabla) \mathbf{u}
$$

so that $\mathbf{t}$ is related to $\mathbf{u}$ via the displacement-traction transfer operator 


$$
\left(S_{m}\right)_{j k}(\nabla)=\nu_{i} \Sigma_{j k}^{(i)}(\nabla), \quad j, k=1,2,3 .
$$

Secondly, at infinity we assume the radiation boundary condition in the form of the limiting absorbing principle (see e.g. [9]). This implies that once a small positive imaginary part is introduced into a wave number, all scattered waves decay at infinity. Thirdly, at the tip of the crack, we impose the so-called tip condition, requiring the elastic energy of the total field to be bounded (see e.g. [10], [11]). Combined with (11) this reduces to the requirement

$$
\mathbf{t}=\mathbf{O}\left(r_{t i p}^{-1 / 2}\right)
$$

where $r_{t i p}$ denotes the dimensionless distance from the point of observation to the tip of the crack.

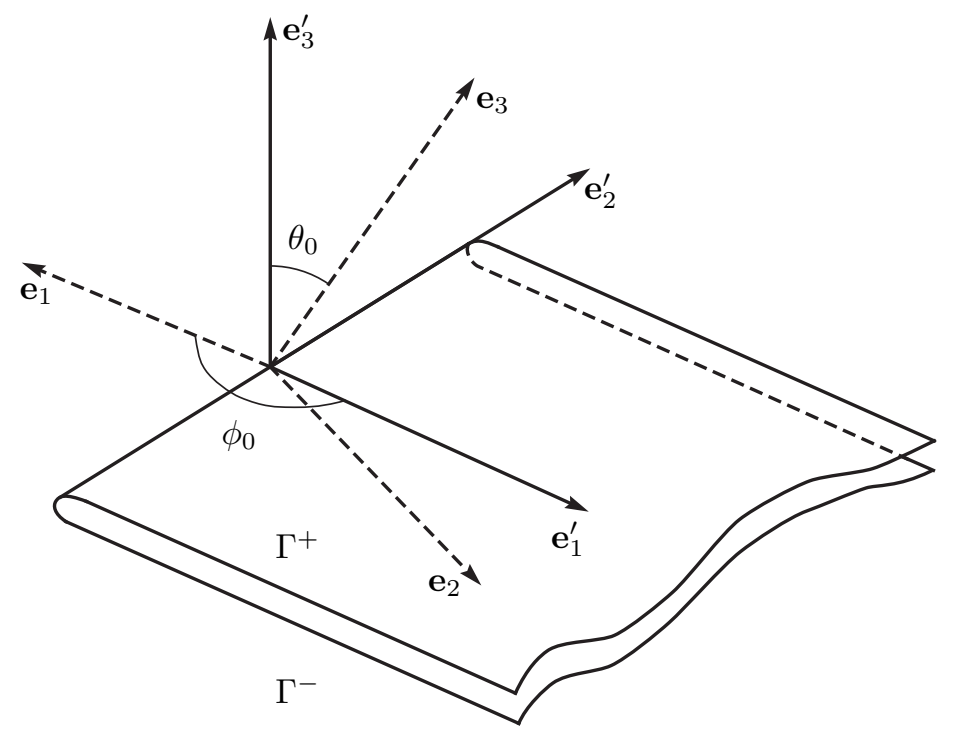

Fig. 1. The geometry of the problem: the half-plane crack with non-contacting faces $\Gamma^{+}$and $\Gamma^{-}$, the medium coordinate system $\left\{\mathbf{e}_{1}, \mathbf{e}_{2}, \mathbf{e}_{3}\right\}$ and the crack coordinate system $\left\{\mathbf{e}_{1}, \dot{\mathbf{e}}_{2}, \dot{\mathbf{e}}_{3}\right\}$.

\section{Transfer Tensor}

Let us introduce the Green's tensor, $u^{G}=u_{m}^{G}(\mathbf{x})$, the solution of the system of elastodynamic equations

$$
L_{i j}(\nabla) u_{j k}^{G}(\mathbf{x})+u_{i k}^{G}(\mathbf{x})=-\delta(\mathbf{x}) \delta_{i k}
$$

where $\delta(\mathbf{x})$ is the delta function and $\delta_{i k}$ is the Kronecker delta. Taking the triple Fourier transform, which is denoted everywhere by the hat ${ }^{-}$, Eq. (17) gives us

$$
\widehat{L}_{m}(\boldsymbol{\xi}) \widehat{u}_{m}^{G}(\boldsymbol{\xi})-\widehat{u}_{m}^{G}(\boldsymbol{\xi})=I
$$


where $I$ is the $3 \times 3$ identity matrix and as above, the subscript $m$ is used to denote a function whose vector argument is expressed in medium coordinates. The solution of (18) is called the transfer tensor.

To continue, the determinant of the so-called Kelvin-Cristoffel matrix $\widehat{L}_{m}(\boldsymbol{\xi})-\lambda_{m}(\boldsymbol{\xi}) I$ can be expressed in the form

$$
\left|\widehat{L}_{m}(\boldsymbol{\xi})-\lambda_{m}(\boldsymbol{\xi}) I\right|=\Delta_{1-2}(\boldsymbol{\xi}) \cdot \Delta_{3}(\boldsymbol{\xi}),
$$

where we employ the notations

$$
\begin{aligned}
& \Delta_{1-2}(\boldsymbol{\xi})=\left[A_{11} \xi_{\perp}^{2}+B_{1} \xi_{3}^{2}-\lambda_{m}(\boldsymbol{\xi})\right]\left[B_{1} \xi_{\perp}^{2}+A_{33} \xi_{3}^{2}-\lambda_{m}(\boldsymbol{\xi})\right]-\left(A_{13}+B_{1}\right)^{2} \xi_{\perp}^{2} \xi_{3}^{2}, \\
& \Delta_{3}(\boldsymbol{\xi})=B_{3} \xi_{\perp}^{2}+B_{1} \xi_{3}^{2}-\lambda_{m}(\boldsymbol{\xi}),
\end{aligned}
$$

with $\xi_{\perp}^{2}=\xi_{1}^{2}+\xi_{2}^{2}$. Thus, eigenvalues of $\widehat{L}_{m}(\boldsymbol{\xi})$, that is zeros of $\Delta_{1-2}$ and $\Delta_{3}$ are

$$
\begin{aligned}
& \lambda_{m}^{(\beta)}(\boldsymbol{\xi})= \\
& \frac{1}{2}\left[\left(B_{1}+A_{11}\right) \xi_{\perp}^{2}+\left(B_{1}+A_{33}\right) \xi_{3}^{2}-\right. \\
& \left.(-1)^{\beta}\left\{\left[\left(A_{11}-B_{1}\right) \xi_{\perp}^{2}+\left(B_{1}-A_{33}\right) \xi_{3}^{2}\right]^{2}+4\left(B_{1}+A_{13}\right)^{2} \xi_{\perp}^{2} \xi_{3}^{2}\right\}^{\frac{1}{2}}\right], \quad \beta=1,2, \\
& \lambda_{m}^{(3)}(\boldsymbol{\xi})=B_{3} \xi_{\perp}^{2}+B_{1} \xi_{3}^{2},
\end{aligned}
$$

and the corresponding unit eigenvectors are

$$
\widehat{\mathbf{p}}_{m}^{(\beta)}(\boldsymbol{\xi})=\frac{\mathbf{V}_{m}^{(\beta)}(\boldsymbol{\xi})}{V_{m}^{(\beta)}(\boldsymbol{\xi})}, \quad \beta=1,2,3
$$

where no summation is implied and we use

$$
\mathbf{V}_{m}^{(\beta)}(\boldsymbol{\xi})=\left(\begin{array}{c}
\left(B_{1}+A_{13}\right) \xi_{1} \xi_{3} \\
\left(B_{1}+A_{13}\right) \xi_{2} \xi_{3} \\
\lambda_{m}^{(\beta)}(\boldsymbol{\xi})-A_{11} \xi_{\perp}^{2}-B_{1} \xi_{3}^{2}
\end{array}\right), \mathbf{V}_{m}^{(3)}(\boldsymbol{\xi})=\left(\begin{array}{c}
-\xi_{2} \\
\xi_{1} \\
0
\end{array}\right)
$$

Note that if $\xi_{2}=0$, as $\xi_{1} \rightarrow 0$, we have

$$
\widehat{\mathbf{p}}_{m}^{(1)}(\boldsymbol{\xi}) \rightarrow \mathbf{e}_{3}, \widehat{\mathbf{p}}_{m}^{(2)}(\boldsymbol{\xi}) \rightarrow \mathbf{e}_{1}, \widehat{\mathbf{p}}_{m}^{(3)}(\boldsymbol{\xi}) \rightarrow \mathbf{e}_{2} .
$$

The unit eigenvectors of $L_{m}(\boldsymbol{\xi})$ form an orthonormal basis, which can be used to expand any function, that is, we can write

$$
\widehat{u}_{m}^{G}(\boldsymbol{\xi})=\mathbf{a}^{(\beta)} \widehat{\mathbf{p}}_{m}^{(\beta)}(\boldsymbol{\xi}),
$$

where the outer product $\mathbf{a b}$ is a tensor with components $(\mathbf{a b})_{i k}=a_{i} b_{k}$. Here and everywhere below, unless stated otherwise, we imply summation over the repeated superscript $\beta=1,2,3$.

Substituting (25) into (18) and using the definition of eigenvectors, we find that the vectors $\mathbf{a}^{(\beta)}$ satisfy the matrix equation 


$$
\left[\lambda_{m}^{(\beta)}(\boldsymbol{\xi})-1\right] \mathbf{a}^{(\beta)} \widehat{\mathbf{p}}_{m}^{(\beta)}(\boldsymbol{\xi})=I .
$$

Dot-multiplying (26) by $\widehat{\mathbf{p}}_{m}^{(\beta)}, \beta=1,2,3$ from the right, we obtain the transfer tensor

$$
\widehat{u}_{m}^{G}(\boldsymbol{\xi})=\frac{\widehat{\mathbf{p}}_{m}^{(\beta)}(\boldsymbol{\xi}) \widehat{\mathbf{p}}_{m}^{(\beta)}(\boldsymbol{\xi})}{\lambda_{m}^{(\beta)}(\boldsymbol{\xi})-1} .
$$

\subsection{Slowness surfaces and wavefronts}

In view of the above, the TI media are conveniently described by the so-called slowness surfaces generated by the slowness vectors $\left[c_{m}^{(\beta)}(\mathbf{n})\right]^{-1} \mathbf{n}$, where $c$ is the wave speed and the unit vector $\mathbf{n}$ indicates the direction of wave propagation [3]. The slowness curves, cross-sections of these surfaces with $\xi_{2}=0$ are shown in Fig. 2 (a). The slowness surfaces can be used to construct the wavefronts of waves radiated by a point source at the origin.

a

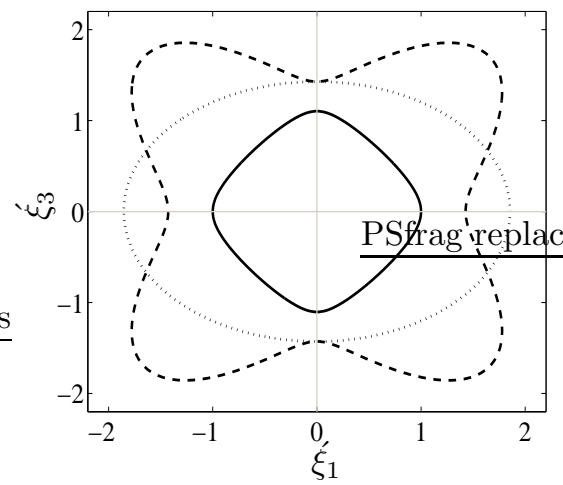

b

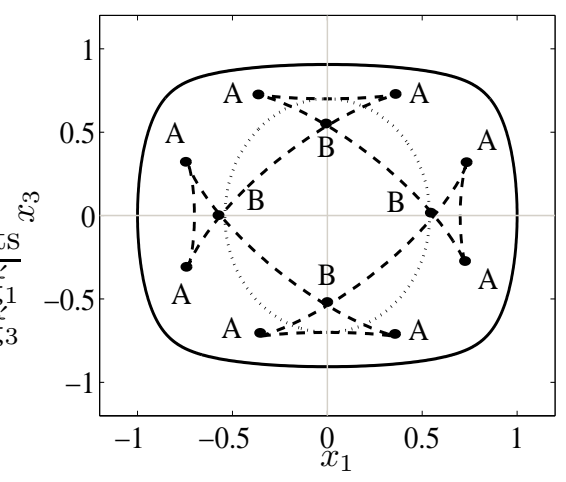

Fig. 2. (a) The slowness curves and (b) wave curves for $\xi_{2}=0$. When the wave curve is rotated around the $x_{3}$ axis, points A circumscribe the so-called cuspidal edges. Points $\mathrm{B}$ are called the conical points. Key: $q P$ (solid line), $q S V$ (dashed line) and $q S H$ (dotted line).

Indeed, the front of each infinitesimal plane wave of type $\beta$ radiated by this source in the direction of a unit vector $\mathbf{n}$ moves to, after a unit of time, the location $\mathbf{x}$ such that we have

$$
\mathbf{n} \cdot \mathbf{x}=c_{m}^{(\beta)}(\mathbf{n}) .
$$

The envelope of the above plane fronts is obtained by differentiating the wavefront equation (28) with respect to $\mathbf{n}$. Therefore, the front of the wave radiated by the point source is generated by the rays

$$
\mathbf{x}=\frac{\partial c_{m}^{(\beta)}(\mathbf{n})}{\partial \mathbf{n}}
$$

The wave curves, the cross-sections of the above wave fronts with $\xi_{2}=0$ are shown in see Fig. 2 (b). Note that the rays indicate the direction of the energy propagation and are perpendicular to the slowness surfaces and not the wavefronts (see Fig. 3 (a)). 


\section{The reciprocity theorem (Green's formula)}

Let us introduce the Cartesian system $\left\{\dot{\mathbf{e}}_{1}, \dot{\mathbf{e}}_{2}, \mathbf{e}_{3}\right\}$ associated with the crack, such that its $\dot{e}_{3}$ axis is perpendicular to the crack plane, $\dot{e}_{2}$ runs along the crack edge and $\dot{\mathbf{e}}_{1}$ is the inner normal to the edge. Note that there exists a matrix of coordinate rotation $Q$, such that $Q^{-1}=Q^{T}$, where the superscript ${ }^{T}$ denotes a transpose, and the medium coordinates $v_{i}$ of any vector $\mathbf{v}=\mathbf{k}, \mathbf{n}, \mathbf{x}, \boldsymbol{\xi}$ etc. are related to its crack coordinates $v_{j}$ via

$$
v_{i}=Q_{i j}^{T} \dot{v}_{j}, i=1,2,3 .
$$

Below, the bold symbol $\mathbf{v}$ is used to denote the triplet of coordinates of vector $\mathbf{v}$ in the primed (crack) coordinate system. When working in the unprimed (medium) coordinate system the symbol $\mathbf{v}$ is retained to denote the triplet of the corresponding coordinates. Since the medium is isotropic in the $\mathbf{e}_{1} \mathbf{e}_{2}$-plane, we can always rotate the medium coordinate system around the axis of symmetry $\mathbf{e}_{3}$ so that $\dot{\mathbf{e}}_{3}$, the normal to the crack plane, lies in the $\mathbf{e}_{2} \mathbf{e}_{3}$-plane and therefore, $\mathbf{e}_{1}$ lies in the crack plane $\mathbf{e}_{1} \mathbf{e}_{2}$. Then without loss of generality, the matrix of coordinate rotation is

$$
Q=\left[\begin{array}{ccc}
\cos \phi_{0} & \sin \phi_{0} \cos \theta_{0} & \sin \phi_{0} \sin \theta_{0} \\
-\sin \phi_{0} & \cos \phi_{0} \cos \theta_{0} & \cos \phi_{0} \sin \theta_{0} \\
0 & -\sin \theta_{0} & \cos \theta_{0}
\end{array}\right],
$$

where $\theta_{0}$ is the angle between the symmetry axis and normal to the crack, and $\phi_{0}$ is the angle between $\mathbf{e}_{1}$ and $\mathbf{e}_{1}$.

We can now use the reciprocity theorem (see e.g [1]) to reformulate the problem stated in Section 2 as an integral equation for the scattered field (otherwise known as the Green's formula). We start by decomposing the total fields $\mathbf{u}$ and $\sigma$ as

$$
\begin{gathered}
\mathbf{u}=\mathbf{u}^{i n}+\mathbf{u}^{s c}, \\
\sigma=\sigma^{i n}+\sigma^{s c},
\end{gathered}
$$

with $\mathbf{u}^{i n}$ and $\sigma^{i n}$ representing the respective incident displacement and stress fields. The incident displacement field can be written as

$$
\mathbf{u}^{i n}(\dot{\mathbf{x}})=\widehat{\mathbf{p}}^{(\beta)}\left(\hat{\mathbf{k}}^{i n}\right) \mathrm{e}^{\mathbf{i} \dot{k}^{i n} \cdot \dot{\mathbf{x}}},
$$

where we use the notation

$$
\mathbf{u}^{i n}(\dot{\mathbf{x}})=\left.\mathbf{u}_{m}^{i n}(\mathbf{x})\right|_{\mathbf{x}=Q^{T} \dot{\mathbf{x}}} ;
$$

the polarization vector $\widehat{\mathbf{p}}^{(\beta)}=\widehat{\mathbf{p}}^{(\beta)}(\dot{\boldsymbol{\xi}})$ is

$$
\widehat{\mathbf{p}}^{(\beta)}(\dot{\boldsymbol{\xi}})=\left.\widehat{\mathbf{p}}_{m}^{(\beta)}(\boldsymbol{\xi})\right|_{\boldsymbol{\xi}=Q^{T} \boldsymbol{\xi}} ;
$$

the wave vector is $\mathbf{k}^{i n}=k^{(\beta)} \mathbf{n}^{i n}$, where $\mathbf{n}^{\text {in }}$ is the propagation direction of the incident wave, given in the primed (crack) coordinates, and the wave number $k^{(\beta)}=\omega / c^{(\beta)}\left(\dot{\mathbf{n}}^{\text {in }}\right)$ is 


$$
k^{i n}=\left[\lambda^{(\beta)}\left(\dot{\mathbf{n}}^{i n}\right)\right]^{-1 / 2},
$$

where similarly to (35) and (36), we use the notation

$$
\lambda^{(\beta)}(\dot{\boldsymbol{\xi}})=\left.\lambda_{m}^{(\beta)}(\boldsymbol{\xi})\right|_{\boldsymbol{\xi}=Q^{T} \dot{\boldsymbol{\xi}}^{\circ}}
$$

We note that the incident stress field gives rise to the incident tractions

$$
\mathbf{t}^{i n}(\dot{\mathbf{x}})=\widehat{\mathbf{t}}^{(\beta)}\left(\hat{\mathbf{k}}^{i n}\right) \mathrm{e}^{i \mathbf{k}^{i n} \cdot \dot{\mathbf{x}}}
$$

and the boundary condition (12) can be rewritten as

$$
\left.\mathbf{t}^{s c}(\dot{\mathbf{x}})\right|_{\Gamma^{ \pm}}=-\left.\mathbf{t}^{i n}(\dot{\mathbf{x}})\right|_{\Gamma^{ \pm}} .
$$

The amplitude of the incident tractions $\widehat{\mathbf{t}}^{(\beta)}\left(\mathbf{k}^{i n}\right)$ is related to the amplitude of the incident displacement field $\widehat{\mathbf{p}}^{(\beta)}$ via

$$
\widehat{\mathbf{t}}^{(\beta)}(\dot{\boldsymbol{\xi}})=\mathrm{i} S(\dot{\boldsymbol{\xi}}) \widehat{\mathbf{p}}^{(\beta)}(\dot{\boldsymbol{\xi}}) .
$$

The latter relationship is obtained by substituting (34) into (14), applying the triple Fourier Transform to the result, changing from $\boldsymbol{\xi}$ to $\boldsymbol{\xi}$ and finally using (15) and the notation

$$
S(\dot{\boldsymbol{\xi}})=\left.S_{m}(\boldsymbol{\xi})\right|_{\boldsymbol{\xi}=Q^{T} \boldsymbol{\xi}^{*}}
$$

As before, the presence (absence) of subscript $m$ means that the function has the vector argument expressed in the medium (crack) coordinates.

By taking into account that both crack faces are traction-free, we then write the reciprocity theorem for the scattered field $\mathbf{u}^{s c}$ as

$$
\mathbf{u}^{s c}(\dot{\mathbf{x}})=-\int_{0}^{\infty} d \dot{y}_{1} \int_{-\infty}^{\infty} d \dot{y}_{2} t^{G}(\dot{\mathbf{x}}-\dot{\mathbf{y}}) \Delta \mathbf{u}^{s c}(\dot{\mathbf{y}})
$$

where $t^{G}=\boldsymbol{\nu} \cdot \sigma^{G}$ is the Green's traction tensor, $\boldsymbol{\nu}=\mathbf{e}_{3}, \sigma_{i j k}^{G}=\Sigma_{j \ell}^{(i)}(\nabla) u_{\ell k}^{G}$ is the Green's stress tensor and $\Delta \mathbf{u}^{s c}\left(\dot{x}_{1}, \dot{x}_{2}\right)$ is the so-called COD (Crack Opening Displacement) (see e.g [1]), which is defined as

$$
\Delta \mathbf{u}^{s c}\left(\dot{x}_{1}, \dot{x}_{2}\right)=\mathbf{u}^{s c}\left(\dot{x}_{1}, \dot{x}_{2}, 0+\right)-\mathbf{u}^{s c}\left(\dot{x}_{1}, \dot{x}_{2}, 0-\right) .
$$

Above, $0+$ refers to the upper face of the crack and $0-$ to the lower.

Let us note that the scattered field is invariant with respect to translations along the edge of the crack. Therefore, all the fields $\mathbf{u}, \mathbf{t}, \sigma$ and $\Delta \mathbf{u}^{s c}$ have a common factor $\exp \left(-\mathrm{i} \dot{\xi}_{2} \dot{x}_{2}\right)$, where we have

$$
\dot{\xi}_{2}=-\dot{k}_{2}^{i n},
$$

and all can be factorized as follows

$$
\mathbf{v}(\dot{\mathbf{x}})=\mathbf{V}\left(\dot{x}_{1}, \dot{x}_{3} ; \dot{\xi}_{2}\right) \mathrm{e}^{-\mathrm{i} \dot{\xi}_{2} \dot{x}_{2}}
$$

Here and everywhere the argument $\xi_{2}$ is separated from other arguments by a semicolumn to emphasize the fact that in the problem under consideration it is just a fixed 
parameter. Also, the bold capitals are used everywhere to denote the preexponential factors of the quantities denoted by the corresponding lower case letters. Then the integral equation (43) can be rewritten as

$$
\begin{aligned}
& \mathbf{U}^{s c}\left(\dot{x}_{1}, \dot{x}_{3} ; \dot{\xi}_{2}\right)= \\
& -\int_{0}^{\infty} d \dot{y}_{1} \int_{-\infty}^{\infty} d \dot{y}_{2} t^{G}\left(\dot{x}_{1}-\dot{y}_{1}, \dot{x}_{2}-\dot{y}_{2}, \dot{x}_{3}\right) \Delta \mathbf{U}^{s c}\left(\dot{y}_{1} ; \dot{\xi}_{2}\right) \mathrm{e}^{\mathrm{i} \dot{\xi}_{2}\left(\dot{x}_{2}-\dot{y}_{2}\right)}
\end{aligned}
$$

where the components of vector functions $\mathbf{U}^{s c}, \Delta \mathbf{U}^{s c}$ and tensor $t^{G}$ are all given in medium coordinates while the crack coordinates are used to represent the components of vector arguments. This choice leads to simpler formulas.

\section{The $3 \mathrm{D}$ functional equation}

Noting that the $\dot{y}_{2}$-integral in (47) is actually a single Fourier transform, which is denoted everywhere by the bar ${ }^{-}$, and applying the convolution theorem to the $\dot{y}_{1}-$ integral, the single Fourier transform of (47) in $\dot{x}_{1}$ gives us the vector functional equation

$$
\overline{\mathbf{U}}^{s c}\left(\dot{\xi}_{1}, \dot{x}_{3} ; \dot{\xi}_{2}\right)=-\widetilde{t}^{G}\left(\dot{\xi}_{1}, \dot{x}_{3} ; \dot{\xi}_{2}\right) \overline{\Delta \mathbf{U}}^{s c}\left(\dot{\xi}_{1} ; \dot{\xi}_{2}\right) .
$$

Above and everywhere below, the tilde $\sim$ denotes the double Fourier transform The tensor $\widetilde{t}^{G}\left(\dot{\xi}_{1}, \dot{x}_{3} ; \xi_{2}\right)$ is the inverse Fourier transform in $\dot{\xi}_{3}$ of the traction tensor $\widehat{t}^{G}(\dot{\boldsymbol{\xi}})$, which is given by

$$
\widehat{t}^{G}(\dot{\boldsymbol{\xi}})=\frac{\widehat{\mathbf{p}}^{(\beta)}(\dot{\boldsymbol{\xi}}) \widehat{\mathbf{t}}^{(\beta)}(\dot{\boldsymbol{\xi}})}{1-\lambda^{(\beta)}(\hat{\boldsymbol{\xi}})} .
$$

This means that we can write

$$
\widetilde{t}^{G}\left(\dot{\xi}_{1}, \dot{x}_{3} ; \dot{\xi}_{2}\right)=\frac{1}{2 \pi} \int_{-\infty}^{\infty} \widehat{t}^{G}(\dot{\boldsymbol{\xi}}) \mathrm{e}^{-\mathrm{i} \dot{\xi}_{3} \dot{x}_{3}} d \dot{\xi}_{3}
$$

Note that as $\dot{\xi}_{3} \rightarrow \infty, \widehat{t}^{G}(\dot{\boldsymbol{\xi}})=O\left(\dot{\xi}_{3}^{-1}\right)$. Therefore, above we can apply the Jordan Lemma if for $\dot{x}_{3}>0+$ the contour of integration is closed in the lower $\dot{\xi}_{3}$-plane and for $\dot{x}_{3}<0-$, in the upper half-plane. In both cases considered below, for each $\xi_{1}$, inside the chosen contour, the Transfer Tensor has two poles $\dot{\xi}_{3}^{(\alpha) \pm}\left(\dot{\xi}_{1} ; \dot{\xi}_{2}\right) \alpha=1$, 2, which are roots of the quartic expression in (20) with the corresponding $\lambda^{(\alpha)}(\dot{\boldsymbol{\xi}})=1$, and one pole $\dot{\xi}_{3}^{(3) \pm}\left(\dot{\xi}_{1} ; \dot{\xi}_{2}\right)$, which is a root of the quadratic expression in (20) with $\lambda^{(3)}(\dot{\boldsymbol{\xi}})=1$. Above, the top sign is chosen when $x_{3}>0+$ and bottom when $x_{3}<0-$; and for the cases under consideration, $\dot{\xi}_{3}^{(\alpha) \pm}\left(\dot{\xi}_{1} ; \dot{\xi}_{2}\right)=\mp \dot{\xi}_{3}^{(\alpha)}\left(\dot{\xi}_{1} ; \dot{\xi}_{2}\right)$; where the functions $\dot{\xi}_{3}^{(\alpha)}\left(\dot{\xi}_{1} ; \dot{\xi}_{2}\right)$ are defined in (59) and (99), respectively. The real parts of functions (59) are shown in Fig. 3 (b). When $\dot{\xi}_{3}^{(\alpha)}\left(\dot{\xi}_{1} ; \dot{\xi}_{2}\right)$ run along slowness surfaces the functions have no imaginary parts. Fig. 3 (b) shows that $\alpha=1$ always describes the $q S V$ mode, $\alpha=3$ - the $q S H$ mode and $\alpha=2$ - the $q P$ or $q S V$ mode, depending on $\xi_{1}$. The functions (99) exhibit analogous behavior.

On adding a small negative imaginary part to all $c^{(\alpha)}$ (a small positive part to the wave numbers), each real pole moves away from the real axis in the $\dot{\xi}_{3}$-plane, with the sign of the resulting $-\operatorname{Im} \dot{\xi}_{3}^{(\alpha) \pm}\left(\xi_{1}, c^{(\alpha)}\right)$ the same as the sign of the partial derivative 
a

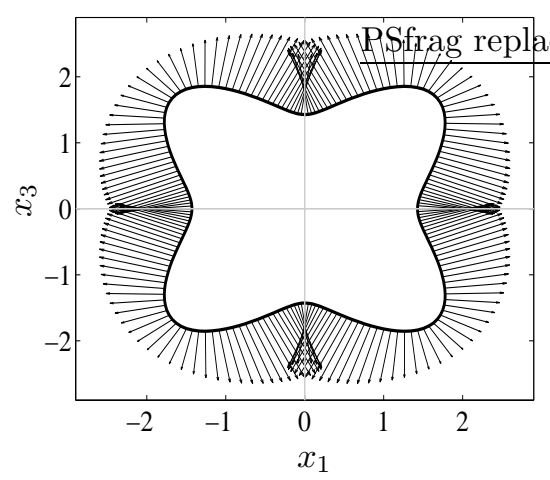

b

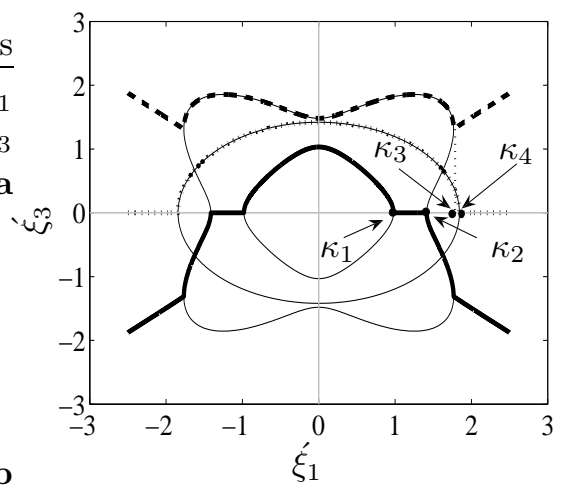

Fig. 3. (a) The $q S V$ energy flow (the mean Poynting) vectors $\mathbf{P}=-\frac{1}{2} \operatorname{Im}\left[\omega \sigma^{d} \cdot\left(\mathbf{u}^{\mathbf{d}}\right)^{*}\right]$, where the star denotes the complex conjugate. (b) The real parts of three roots $\dot{\xi}_{3}^{(1)}\left(\dot{\xi}_{1} ; \dot{\xi}_{2}\right)$ (dashed line), $\dot{\xi}_{3}^{(2)}\left(\dot{\xi}_{1} ; \dot{\xi}_{2}\right)$ (solid line) and $\dot{\xi}_{3}^{(3)}\left(\dot{\xi}_{1} ; \xi_{2}\right)$ (dotted line) imposed on the slowness curves in Fig. 2 (a). When lying on the slowness curves the roots are real. When sliding of these curves at the branch points $\xi_{1}=\kappa_{1}, \kappa_{2}, \kappa_{3}$ or $\kappa_{4}$ the roots are complex.

$\partial \dot{\xi}_{3}^{(\alpha) \pm}\left(\xi_{1}, c^{(\alpha)}\right) / \partial c^{(\alpha)}$. In other words, the resulting $-\operatorname{Im} \dot{\xi}_{3}^{(\alpha) \pm}\left(\xi_{1}, c^{(\alpha)}\right)$ has the same sign as the vertical component of the gradient to the slowness surface at $\xi_{1}$ (see Figs. 2 (a) and $3(\mathrm{~b})$ ), that is the sign of the vertical component of the energy flux (see Fig. 3 (a)). Since $\widehat{t}^{G}(\hat{\boldsymbol{\xi}})$ in (49) is a rational function of $\xi_{3}$ we can now evaluate the integral (50) using the Cauchy Residue Theorem to obtain

$$
\widetilde{t}^{G}\left(\dot{\xi}_{1}, \dot{x}_{3} ; \dot{\xi}_{2}\right)=\left.\mp \mathrm{i} \frac{\widehat{\mathbf{p}}^{(\alpha)}(\dot{\boldsymbol{\xi}}) \widehat{\mathbf{t}}^{(\alpha)}(\dot{\boldsymbol{\xi}})}{\lambda_{\dot{\xi}_{3}}^{(\alpha)}(\dot{\boldsymbol{\xi}})}\right|_{\xi_{3}=\dot{\xi}_{3}^{(\alpha) \pm}}{\left(\xi_{1} ; \xi_{2}\right) ; \lambda(\alpha)\left(\dot{\boldsymbol{\xi}}_{)=1}\right.} \mathrm{e}^{-\mathrm{i} \dot{\xi}_{3}^{(\alpha) \pm}\left(\dot{\xi}_{1} ; \dot{\xi}_{2}\right) \dot{x}_{3}}
$$

where the subscript $\dot{\xi}_{3}$ indicates differentiation with respect to $\dot{\xi}_{3}$ and the resulting plane waves satisfy the radiation condition at infinity. For the two cases considered in this paper the denominators in (51) are specified below in (124) and (125), respectively. We also note that $\widetilde{t}^{G}\left(\dot{\xi}_{1}, 0-; \dot{\xi}_{2}\right)-\widetilde{t}^{G}\left(\dot{\xi}_{1}, 0+; \dot{\xi}_{2}\right)=I$, the identity matrix. This can be best seen by using (48) to evaluate $\widetilde{\mathbf{U}}^{s c}\left(\dot{\xi}_{1}, 0+; \dot{\xi}_{2}\right)-\widetilde{\mathbf{U}}^{s c}\left(\dot{\xi}_{1}, 0-; \dot{\xi}_{2}\right)$.

In order to utilize the boundary condition (12) in a straightforward manner, we apply the operator $-\mathrm{i} S\left(\dot{\xi}_{1}, \dot{\xi}_{2}, \mathrm{i} \dot{\partial}_{3}\right)$ to Eq. (48) and then write the resulting equation for the crack face $\dot{x}_{3}=0$ to obtain

$$
\overline{\mathbf{T}}^{s c}\left(\dot{\xi}_{1}, 0 ; \dot{\xi}_{2}\right)=-\widetilde{\tau}^{G}\left(\dot{\xi}_{1}, 0 ; \dot{\xi}_{2}\right) \overline{\Delta \mathbf{U}}^{s c}\left(\dot{\xi}_{1} ; \dot{\xi}_{2}\right),
$$

where $S\left(\dot{\xi}_{1}, \dot{\xi}_{2}, \mathrm{i} \partial_{3}\right)=S(\dot{\xi})$, with i $\partial / \partial \dot{x}_{3}$ substituted for $\dot{\xi}_{3}$, and we have

$$
\widetilde{\tau}^{G}\left(\dot{\xi}_{1}, \dot{x}_{3} ; \dot{\xi}_{2}\right)= \pm\left.\mathrm{i} \frac{\widehat{\mathbf{t}}^{(\alpha)}(\dot{\boldsymbol{\xi}}) \widehat{\mathbf{t}}^{(\alpha)}(\dot{\boldsymbol{\xi}})}{\lambda_{\dot{\xi}_{3}}^{(\alpha)}(\dot{\boldsymbol{\xi}})}\right|_{\dot{\xi}_{3}=\dot{\xi}_{3}^{(\alpha) \pm}}{\left(\dot{\xi}_{1} ; \dot{\xi}_{2}\right) ; \lambda^{(\alpha)}\left(\dot{\boldsymbol{\xi}}_{)=1}\right.} \mathrm{e}^{-\mathrm{i} \dot{\xi}_{3}^{(\alpha) \pm}\left(\dot{\xi}_{1} ; \dot{\xi}_{2}\right) \dot{x}_{3}}
$$

Note that on the crack plane, $\mathbf{T}^{s c}$ must be a continuous function of $\mathbf{x}$, so that $\overline{\mathbf{T}}^{s c}\left(\dot{\xi}_{1}, 0+; \dot{\xi}_{2}\right)=$ $\overline{\mathbf{T}}^{s c}\left(\dot{\xi}_{1}, 0-; \dot{\xi}_{2}\right)$ and therefore, $\widetilde{\tau}^{G}\left(\dot{\xi}_{1}, 0 ; \dot{\xi}_{2}\right)$ is well-defined. This can be verified independently by expanding $\widehat{\tau}_{i j}^{G}(\dot{\xi})$, the inverse Fourier transform in $\dot{\xi}_{3}$ of (53), into the Laurents 
series, to verify that for any crack orientation, its components exhibit the following behavior at infinity:

$$
\widehat{\tau}_{i j}^{G}(\dot{\boldsymbol{\xi}})=\text { constant matrix }+O\left(\frac{1}{\dot{\xi}_{3}^{2}}\right) \text {, as } \dot{\xi}_{3} \rightarrow \infty .
$$

Then $\widetilde{\tau}^{G}\left(\dot{\xi}_{1}, 0 ; \dot{\xi}_{2}\right)$ is well-defined via the following consideration:

$$
\widetilde{\tau}^{G}\left(\dot{\xi}_{1}, 0+; \dot{\xi}_{2}\right)-\widetilde{\tau}^{G}\left(\dot{\xi}_{1}, 0-; \dot{\xi}_{2}\right)=\lim _{R \rightarrow \infty} \oint \widehat{\tau}^{G}(\dot{\boldsymbol{\xi}}) d \dot{\xi}_{3}=0,
$$

where the integration contour is a circle of radius $R$, circumscribed anticlockwise and centered at the origin of coordinates. In view of the above, below we use the simplified notations

$$
\mathbf{T}^{s c}\left(\dot{x}_{1} ; \dot{\xi}_{2}\right)=\mathbf{T}^{s c}\left(\dot{x}_{1}, 0 ; \dot{\xi}_{2}\right), \quad \widetilde{\tau}^{G}\left(\dot{\xi}_{1} ; \dot{\xi}_{2}\right)=\widetilde{\tau}^{G}\left(\dot{\xi}_{1}, 0 ; \dot{\xi}_{2}\right),
$$

We can now make use of the boundary conditions (40) and apply the Fourier Transform to (39) to put the functional equation (52) in the Wiener-Hopf form,

$$
\mathrm{i} \frac{\widehat{\mathbf{t}}^{(\beta)}\left(\dot{\mathbf{k}}^{i n}\right)}{\dot{\xi}_{1}+\dot{k}_{1}^{i n}+i 0}-\overline{\mathbf{T}}^{s c-}\left(\dot{\xi}_{1} ; \dot{\xi}_{2}\right)=\widetilde{\tau}^{G}\left(\dot{\xi}_{1} ; \dot{\xi}_{2}\right) \overline{\Delta \mathbf{U}}^{s c}\left(\dot{\xi}_{1} ; \dot{\xi}_{2}\right),
$$

where $\overline{\Delta \mathbf{U}^{s c}}\left(\xi_{1} ; \xi_{2}\right)$ and $\overline{\mathbf{T}}^{s c-}\left(\dot{\xi}_{1} ; \xi_{2}\right)$ are both unknown. Since $\Delta \mathbf{U}^{s c}\left(\dot{x}_{1} ; \dot{x}_{2}\right)=\mathbf{0}$ and $\mathbf{T}^{s c-}\left(\dot{x}_{1} ; \dot{x}_{2}\right)=\mathbf{0}$ for $\dot{x}_{1} \leq 0$ and $\dot{x}_{1} \geq 0$, respectively, their Fourier transforms $\overline{\Delta \mathbf{U}^{s c}}\left(\dot{\xi}_{1} ; \dot{\xi}_{2}\right)$ and $\overline{\mathbf{T}}^{s c-}\left(\dot{\xi}_{1} ; \dot{\xi}_{2}\right)$ are analytic in the upper and lower half of the complex $\dot{\xi}_{1}$-plane, respectively. This can be seen by studying the corresponding single Fourier integral. Above, we also use the fact that we have

$$
\int_{0}^{\infty} \mathrm{e}^{\mathrm{i}\left[\dot{\xi}_{1}+\dot{k}_{1}^{i n}\right] \dot{x}_{1}} d \dot{x}_{1}=\frac{\mathrm{i}}{\dot{\xi}_{1}+\dot{k}_{1}^{i n}+i 0} .
$$

\section{Solution of the 3D functional equation when the symmetry axis is perpendicular to the crack plane}

\subsection{The poles and branch points of $\hat{t}^{G}(\boldsymbol{\xi})$}

When the symmetry axis is perpendicular to the crack plane, $\phi_{0}=\theta_{0}=0, Q=I$ and thus, all the $\xi$-components coincide with the corresponding $\dot{\xi}$-components. As mentioned above, the functions $\pm \dot{\xi}_{3}^{(\alpha)}\left(\dot{\xi}_{1} ; \dot{\xi}_{2}\right), \alpha=1,2$ and $\pm \dot{\xi}_{3}^{(3)}\left(\dot{\xi}_{1} ; \dot{\xi}_{2}\right)$ which are the poles of the tensor $\hat{t}^{G}(\hat{\boldsymbol{\xi}})$ are the respective roots of the quartic and quadratic expression in (20), with $\lambda^{(\alpha)}(\dot{\boldsymbol{\xi}})=1$, so that we have

$$
\begin{aligned}
& \dot{\xi}_{3}^{(1)}\left(\dot{\xi}_{1} ; \dot{\xi}_{2}\right)=\dot{\xi}_{31}\left(\dot{\xi}_{1} ; \dot{\xi}_{2}\right)+\dot{\xi}_{32}\left(\dot{\xi}_{1} ; \dot{\xi}_{2}\right), \\
& \dot{\xi}_{3}^{(2)}\left(\dot{\xi}_{1} ; \dot{\xi}_{2}\right)=\dot{\xi}_{31}\left(\dot{\xi}_{1} ; \dot{\xi}_{2}\right)-\dot{\xi}_{32}\left(\dot{\xi}_{1} ; \dot{\xi}_{2}\right), \\
& \dot{\xi}_{3}^{(3)}\left(\dot{\xi}_{1} ; \dot{\xi}_{2}\right)=\left(B_{1}^{-1} B_{3}\right)^{1 / 2} \gamma_{4}\left(\dot{\xi}_{1} ; \dot{\xi}_{2}\right),
\end{aligned}
$$

with 


$$
\begin{aligned}
& \dot{\xi}_{3 n}\left(\dot{\xi}_{1} ; \dot{\xi}_{2}\right)=\frac{\gamma_{6-3 n}}{2\left(B_{1} A_{33}\right)^{1 / 2}} \times \\
& \left\{\frac{\left[A_{13}^{2}+2 B_{1} A_{13}-A_{11} A_{33}\right] \xi_{\perp}^{2}+B_{1}+A_{33}-(-1)^{n} 2 B_{1}\left(A_{11} A_{33}\right)^{1 / 2} \gamma_{1} \gamma_{2}}{\gamma_{6-3 n}^{2}}\right\}^{1 / 2}, \\
& n=1,2,
\end{aligned}
$$

\{\}$^{1 / 2}$ denoting the principal value,

$$
\begin{aligned}
& \gamma_{0}=\gamma_{0}\left(\dot{\xi}_{1} ; \dot{\xi}_{2}\right)=\left(\kappa_{0}^{2}+\dot{\xi}_{1}^{2}\right)^{1 / 2} \\
& \gamma_{\ell}=\gamma_{\ell}\left(\dot{\xi}_{1} ; \dot{\xi}_{2}\right)=\left[\left(\kappa_{\ell}+\mathrm{i} 0\right)^{2}-\dot{\xi}_{1}^{2}\right]^{1 / 2}, \quad \ell=1,2,3,4,
\end{aligned}
$$

with the branch points $\pm \mathrm{i} \kappa_{0}$ and $\pm \kappa_{\ell}, \ell=1,2,3,4$, where we have

$$
\begin{aligned}
& \kappa_{0}=\left(C_{0}^{2}+\xi_{2}^{2}\right)^{1 / 2}, \\
& \kappa_{1}=\left(A_{11}^{-1}-\xi_{2}^{2}\right)^{1 / 2}, \\
& \kappa_{2}=\left(B_{1}^{-1}-\xi_{2}^{2}\right)^{1 / 2}, \\
& \kappa_{3}=\left(C_{3}^{2}-\xi_{2}^{2}\right)^{1 / 2}, \\
& \kappa_{4}=\left(B_{3}^{-1}-\xi_{2}^{2}\right)^{1 / 2}
\end{aligned}
$$

and

$$
\begin{aligned}
& C_{\ell}=D^{-1}\left\{2\left(A_{33} B_{1}\right)^{1 / 2}\left(B_{1}+A_{13}\right)\left[A_{13}^{2}-A_{11} A_{33}+B_{1}\left(A_{11}+2 A_{13}+A_{33}\right)\right]^{1 / 2}-\right. \\
& \left.(-1)^{\ell}\left[A_{13}\left(2 B_{1}+A_{13}\right)\left(B_{1}+A_{33}\right)+A_{33}\left(2 B_{1}^{2}+A_{11} B_{1}-A_{11} A_{33}\right)\right]\right\}^{1 / 2}, \quad \ell=0,3, \\
& D=\left\{\left(A_{11} A_{33}-A_{13}^{2}\right)\left[\left(2 B_{1}+A_{13}\right)^{2}-A_{11} A_{33}\right]\right\}^{1 / 2} .
\end{aligned}
$$

It can be checked, e.g. using MATHEMATICA, that when $n=2(n=1), \pm \mathrm{i} \kappa_{0}\left( \pm \kappa_{3}\right)$ is a zero of the corresponding numerator in the curly brackets in (60). Therefore, the square roots of the expressions in the curly brackets never vanish. Note that all branch points are either real or purely imaginary and for the austenitic steels that are described e.g. in [8] and are considered in this article, direct evaluation shows that we have the following inequalities:

$$
\begin{aligned}
& \operatorname{Re} \kappa_{1} \leq \operatorname{Re} \kappa_{2} \leq \operatorname{Re} \kappa_{3} \leq \operatorname{Re} \kappa_{4}, \\
& \operatorname{Im} \kappa_{4} \leq \operatorname{Im} \kappa_{3} \leq \operatorname{Im} \kappa_{2} \leq \operatorname{Im} \kappa_{1} \leq \kappa_{0} .
\end{aligned}
$$

Therefore, the phases of the principal values of $\dot{\xi}_{32}$ and $\dot{\xi}_{31}$ are determined by the position of $\dot{\xi}_{1}$ on the complex $\dot{\xi}_{1}$-plane relative to $\kappa_{2}$ and $\kappa_{3}$. For a fixed values of $\dot{\xi}_{2}$, the real parts of functions $\dot{\xi}_{3}^{(\alpha)}, \alpha=1,2,3$ described by equations (59) are represented in Fig. 3 (b).

\subsection{Decoupling the normal COD from the tangential COD}

In the case under consideration, $t_{i}^{\prime}=\sigma_{i 3}^{\prime}$ and the crack and medium coordinates coincide. Therefore, in view of (42) the transfer operator $S(\xi)$ is 


$$
S(\dot{\boldsymbol{\xi}})=\left[\begin{array}{ccc}
B_{1} \dot{\xi}_{3} & 0 & B_{1} \dot{\xi}_{1} \\
0 & B_{1} \dot{\xi}_{3} & B_{1} \dot{\xi}_{2} \\
A_{13} \dot{\xi}_{1} & A_{13} \dot{\xi}_{2} & A_{33} \dot{\xi}_{3}
\end{array}\right] .
$$

It can be checked, e.g. using MATHEMATICA, that we can write

$$
\widetilde{\tau}^{G}\left(\dot{\xi}_{1} ; \dot{\xi}_{2}\right)=\left[\begin{array}{cc}
\widetilde{\tau}^{r e d}\left(\dot{\xi}_{1} ; \dot{\xi}_{2}\right) & 0 \\
0 & \mu_{3}\left(\dot{\xi}_{1} ; \dot{\xi}_{2}\right)
\end{array}\right]
$$

where we have

$$
\mu_{3}\left(\dot{\xi}_{1} ; \dot{\xi}_{2}\right)=\left(\frac{A_{33}}{A_{11}}\right)^{1 / 2} \frac{\gamma_{2}\left(\dot{\xi}_{1} ; \dot{\xi}_{2}\right)}{\gamma_{1}\left(\dot{\xi}_{1} ; \dot{\xi}_{2}\right)} \mu_{1}\left(\dot{\xi}_{1} ; \dot{\xi}_{2}\right)
$$

and

$$
\widetilde{\tau}^{\text {red }}\left(\dot{\xi}_{1} ; \dot{\xi}_{2}\right)=P^{-1}\left(\dot{\xi}_{1} ; \dot{\xi}_{2}\right) M\left(\dot{\xi}_{1} ; \dot{\xi}_{2}\right) P\left(\dot{\xi}_{1} ; \dot{\xi}_{2}\right),
$$

with

$$
\begin{aligned}
& P\left(\dot{\xi}_{1} ; \dot{\xi}_{2}\right)=\left[\begin{array}{cc}
\dot{\xi}_{1} & \dot{\xi}_{2} \\
\dot{\xi}_{2} & -\dot{\xi}_{1}
\end{array}\right], \\
& M\left(\dot{\xi}_{1} ; \dot{\xi}_{2}\right)=\left[\begin{array}{cc}
\mu_{1}\left(\dot{\xi}_{1} ; \dot{\xi}_{2}\right) & 0 \\
0 & \mu_{2}\left(\dot{\xi}_{1} ; \dot{\xi}_{2}\right)
\end{array}\right], \\
& \mu_{1}\left(\dot{\xi}_{1} ; \dot{\xi}_{2}\right)=\frac{\mathrm{i} R\left(\dot{\xi}_{1} ; \dot{\xi}_{2}\right)}{4 A_{33} \dot{\xi}_{31}\left(\dot{\xi}_{1} ; \dot{\xi}_{2}\right)}, \\
& \mu_{2}\left(\dot{\xi}_{1} ; \dot{\xi}_{2}\right)=\frac{1}{2}\left(-B_{1} B_{3}\right)^{1 / 2} \gamma_{4}\left(\dot{\xi}_{1} ; \dot{\xi}_{2}\right),
\end{aligned}
$$

and the Rayleigh function is defined by

$$
R\left(\dot{\xi}_{1} ; \dot{\xi}_{2}\right)=\left(A_{13}^{2}-A_{11} A_{33}\right)\left(\dot{\xi}_{1}^{2}+\dot{\xi}_{2}^{2}\right)+A_{33}+\left(A_{11} A_{33}\right)^{1 / 2} \frac{\gamma_{1}\left(\dot{\xi}_{1} ; \dot{\xi}_{2}\right)}{\gamma_{2}\left(\dot{\xi}_{1} ; \dot{\xi}_{2}\right)} .
$$

Then substituting (66) into the 3D vector functional equation (57), the latter decouples into the scalar equation describing the COD component normal to the crack face and 2D vector functional equation describing the tangential COD components,

$$
\mu_{3}\left(\dot{\xi}_{1} ; \dot{\xi}_{2}\right) \overline{\Delta U}_{3}^{s c}\left(\dot{\xi}_{1} ; \dot{\xi}_{2}\right)=\mathrm{i} \frac{\widehat{t}_{3}^{(\beta)}\left(\dot{\mathbf{k}}^{i n}\right)}{\dot{\xi}_{1}+\dot{k}_{1}^{i n}+\mathrm{i} 0}-\bar{T}_{3}^{s c-}\left(\dot{\xi}_{1} ; \dot{\xi}_{2}\right)
$$

and 


$$
M\left(\dot{\xi}_{1} ; \dot{\xi}_{2}\right) \overline{\Delta \mathcal{U}}^{s c}\left(\dot{\xi}_{1} ; \dot{\xi}_{2}\right)=\mathrm{i} \frac{\hat{\boldsymbol{\tau}}^{(\beta)}\left(\dot{\mathbf{k}}^{i n}\right)}{\dot{\xi}_{1}+\dot{k}_{1}^{i n}+i 0}-\overline{\mathcal{T}}^{s c-}\left(\dot{\xi}_{1} ; \dot{\xi}_{2}\right),
$$

where the calligraphic script is used to denote two dimensional vectors expanded in the eigenvectors of $\widetilde{\tau}^{\text {red }}$, so that for any vector $\mathcal{A}=\overline{\Delta \mathcal{U}}^{s c}, \overline{\mathcal{T}}^{s c-}$ or $\hat{\boldsymbol{\tau}}^{(\beta)}$ we have

$$
\mathcal{A}_{j}\left(\dot{\xi}_{1} ; \dot{\xi}_{2}\right)=P_{j k}\left(\dot{\xi}_{1} ; \dot{\xi}_{2}\right) A_{k}\left(\dot{\xi}_{1} ; \dot{\xi}_{2}\right), \quad j, k=1,2,
$$

where we remind the reader that the repeated index summation is applied to $k=1,2$.

\subsection{Solving the scalar functional equation for the normal COD}

First, let us consider the scalar equation (73). It can be solved using the Wiener-Hopf technique by introducing the following factorization of the tangential traction $\mu_{3}$ :

$$
\mu_{3}\left(\dot{\xi}_{1} ; \dot{\xi}_{2}\right)=\mu_{3}^{+}\left(\dot{\xi}_{1} ; \dot{\xi}_{2}\right) \mu_{3}^{-}\left(\dot{\xi}_{1} ; \dot{\xi}_{2}\right)
$$

where $+(-)$ means that the function is analytic in the upper (lower) half of the complex $\dot{\xi}_{1}$-plane. In order to effect this factorization, we first note that there exist values $\dot{\xi}_{1}=$ $\pm \kappa_{R}, \kappa_{R}=\left(k_{R}^{2}-\xi_{2}^{2}\right)^{1 / 2}$ such that

$$
R\left( \pm \kappa_{R} ; \dot{\xi}_{2}\right)=0
$$

which are called the Rayleigh poles. Above, $k_{R}=c_{0} / c_{R}$, where $c_{R}$ is the speed of the surface Rayleigh wave. Only the pole $-\kappa_{R}$ corresponds to the outgoing wave. Then the considerations and notations introduced in Appendices A and B allow us to define $\mu_{1}^{ \pm}\left(\dot{\xi}_{1} ; \dot{\xi}_{2}\right)$,

$$
\mu_{1}^{ \pm}\left(\dot{\xi}_{1} ; \dot{\xi}_{2}\right)=\left(\frac{\mathrm{i} l_{0}}{4 l_{1} A_{33}}\right)^{1 / 2} \frac{K_{0}^{ \pm}\left(\dot{\xi}_{1} ; \dot{\xi}_{2}\right)}{\gamma_{3}^{ \pm}\left(\dot{\xi}_{1} ; \dot{\xi}_{2}\right) K_{1}^{ \pm}\left(\dot{\xi}_{1} ; \dot{\xi}_{2}\right)}\left(\kappa_{R} \pm \dot{\xi}_{1}\right),
$$

and therefore, $\mu_{3}^{ \pm}\left(\dot{\xi}_{1} ; \xi_{2}\right)$,

$$
\mu_{3}^{ \pm}\left(\dot{\xi}_{1} ; \dot{\xi}_{2}\right)=\left(\frac{A_{33}}{A_{11}}\right)^{1 / 4} \frac{\gamma_{2}^{ \pm}\left(\dot{\xi}_{1} ; \dot{\xi}_{2}\right)}{\gamma_{1}^{ \pm}\left(\dot{\xi}_{1} ; \dot{\xi}_{2}\right)} \mu_{1}^{ \pm}\left(\dot{\xi}_{1} ; \dot{\xi}_{2}\right)
$$

where throughout we use the notations

$$
\begin{aligned}
& \gamma_{0}^{ \pm}\left(\dot{\xi}_{1} ; \dot{\xi}_{2}\right)=\left(\mathrm{i} \kappa_{0} \pm \dot{\xi}_{1}\right)^{1 / 2}, \\
& \gamma_{\ell}^{ \pm}\left(\dot{\xi}_{1} ; \dot{\xi}_{2}\right)=\left(\kappa_{\ell} \pm \dot{\xi}_{1}\right)^{1 / 2}, \ell=1, \ldots, 4 .
\end{aligned}
$$

To continue, as mentioned at the end of Section $5, \overline{\Delta \mathbf{U}}^{s c}\left(\dot{\xi}_{1} ; \dot{\xi}_{2}\right)$ is analytic in the upper half of the $\dot{\xi}_{1}$-plane. Hence Eq. (73) can be rewritten as

$$
-\mu_{3}^{+}\left(\dot{\xi}_{1} ; \dot{\xi}_{2}\right) \overline{\Delta U}_{3}^{s c}\left(\dot{\xi}_{1} ; \dot{\xi}_{2}\right)+\mathrm{i} \frac{\widehat{t}_{3}^{(\beta)}\left(\hat{\mathbf{k}}^{i n}\right)}{\left(\dot{\xi}_{1}+\dot{k}_{1}^{i n}+i 0\right) \mu_{3}^{-}\left(-\dot{k}_{1}^{i n} ; \dot{\xi}_{2}\right)}=\bar{T}_{3}^{\text {mod- }},
$$

where the superscript ${ }^{\text {mod }}$ stands for modified and we have 


$$
\bar{T}_{3}^{\text {mod- }}=\frac{\bar{T}_{3}^{s c-}\left(\dot{\xi}_{1} ; \dot{\xi}_{2}\right)}{\mu_{3}^{-}\left(\dot{\xi}_{1} ; \dot{\xi}_{2}\right)}+\mathrm{i}\left[\frac{1}{\mu_{3}^{-}\left(-\dot{k}_{1}^{i n} ; \dot{\xi}_{2}\right)}-\frac{1}{\mu_{3}^{-}\left(\dot{\xi}_{1} ; \dot{\xi}_{2}\right)}\right] \frac{\widehat{t}_{3}^{(\beta)}\left(\dot{\mathbf{k}}^{i n}\right)}{\dot{\xi}_{1}+\dot{k}_{1}^{i n}+i 0} .
$$

Note that the modification leading to $\bar{T}_{3}^{\text {mod- }}$ has been introduced to assure that the left-hand (right-hand) side of (81) is analytic in the upper (lower) half of the complex $\xi_{1}$-plane. This can be true only if both sides are one and the same entire function. Furthermore it is easy to check that at infinity we have

$$
\mu_{3}^{+}\left(\dot{\xi}_{1} ; \dot{\xi}_{2}\right)=O\left(\dot{\xi}_{1}^{1 / 2}\right)
$$

Also, the tip condition (16) implies that $\Delta \mathbf{U}^{s c}\left(\hat{x}_{1}, \hat{x}_{2}\right)=\mathbf{O}\left(\hat{x}_{1}^{1 / 2}\right)$, which in its turn implies

$$
\overline{\Delta \mathbf{U}}^{s c}\left(\dot{\xi}_{1} ; \dot{\xi}_{2}\right)=\mathbf{O}\left(\dot{\xi}_{1}^{-3 / 2}\right) .
$$

It follows that as $\dot{\xi}_{1} \rightarrow \infty$, the left-hand side of Eq. (81) and therefore, its right-hand side are both functions of order $O\left(\left[\dot{\xi}_{1}\right]^{-1}\right)$, obviously bounded. According to the Liouville's Theorem, any such entire function is in fact zero. Therefore, Eq. (81) implies

$$
\overline{\Delta U}_{3}^{s c}\left(\dot{\xi}_{1} ; \dot{\xi}_{2}\right)=\mathrm{i} \frac{\widehat{t}_{3}^{(\beta)}\left(\dot{\mathbf{k}}^{i n}\right)}{\left(\dot{\xi}_{1}+\dot{k}_{1}^{i n}+i 0\right) \mu_{3}^{+}\left(\dot{\xi}_{1} ; \dot{\xi}_{2}\right) \mu_{3}^{-}\left(-\dot{k}_{1}^{i n} ; \dot{\xi}_{2}\right)} .
$$

\subsection{Solving the 2D vector functional equation for the tangential COD components}

Let us now turn to the $2 \mathrm{D}$ vector functional equation (74). The matrix $M$ can be factorized,

$$
M\left(\dot{\xi}_{1} ; \dot{\xi}_{2}\right)=M^{+}\left(\dot{\xi}_{1} ; \dot{\xi}_{2}\right) M^{-}\left(\dot{\xi}_{1} ; \dot{\xi}_{2}\right),
$$

so that the matrix

$$
M^{+}\left(\dot{\xi}_{1} ; \dot{\xi}_{2}\right)=\left[\begin{array}{cc}
\mu_{1}^{+}\left(\dot{\xi}_{1} ; \dot{\xi}_{2}\right) & 0 \\
0 & \mu_{2}^{+}\left(\dot{\xi}_{1} ; \dot{\xi}_{2}\right)
\end{array}\right]
$$

is analytic in the upper half of the complex $\xi_{1}$-plane, and matrix

$$
M^{-}\left(\dot{\xi}_{1} ; \dot{\xi}_{2}\right)=\left[\begin{array}{cc}
\mu_{1}^{-}\left(\dot{\xi}_{1} ; \dot{\xi}_{2}\right) & 0 \\
0 & \mu_{2}^{-}\left(\dot{\xi}_{1} ; \dot{\xi}_{2}\right)
\end{array}\right]
$$

is analytic and has no zeros in the lower half of this plane. The factorization of $\mu_{1}\left(\dot{\xi}_{1} ; \dot{\xi}_{2}\right)$ has been described in the previous section, and $\mu_{2}$ can be readily factorized into the factors

$$
\mu_{2}^{ \pm}\left(\dot{\xi}_{1} ; \dot{\xi}_{2}\right)=\left(-\frac{B_{1} B_{3}}{4}\right)^{1 / 4} \gamma_{4}^{ \pm}\left(\dot{\xi}_{1} ; \dot{\xi}_{2}\right) .
$$

Below we also use the matrices $N^{ \pm}$which are respective inverses of $M^{ \pm}$. Now, multiplying (74) by matrix $N^{-}\left(\dot{\xi}_{1} ; \dot{\xi}_{2}\right)$ gives us 


$$
M^{+}\left(\dot{\xi}_{1} ; \dot{\xi}_{2}\right) \overline{\Delta \mathcal{U}}^{s c}\left(\dot{\xi}_{1} ; \dot{\xi}_{2}\right)=\mathrm{i} N^{-}\left(\dot{\xi}_{1} ; \dot{\xi}_{2}\right) \frac{\hat{\boldsymbol{\tau}}^{(\beta)}\left(\dot{\mathbf{k}}^{i n}\right)}{\dot{\xi}_{1}+\dot{k}_{1}^{i n}+i 0}-N^{-}\left(\dot{\xi}_{1} ; \dot{\xi}_{2}\right) \overline{\mathcal{T}}^{s c-}\left(\dot{\xi}_{1} ; \dot{\xi}_{2}\right)
$$

It can be transformed into

$$
-M^{+}\left(\dot{\xi}_{1} ; \dot{\xi}_{2}\right) \overline{\Delta \mathcal{U}}^{s c}\left(\dot{\xi}_{1} ; \dot{\xi}_{2}\right)+\mathrm{i} N^{-}\left(-\dot{k}_{1}^{i n} ; \dot{\xi}_{2}\right) \frac{\hat{\boldsymbol{\tau}}^{(\beta)}\left(\dot{\mathbf{k}}^{i n}\right)}{\xi_{1}+\dot{k}_{1}^{i n}+i 0}=\overline{\mathcal{T}}^{\text {mod }-}\left(\dot{\xi}_{1} ; \dot{\xi}_{2}\right),
$$

where we have

$$
\begin{aligned}
& \overline{\mathcal{T}}^{\text {mod }-}\left(\dot{\xi}_{1} ; \dot{\xi}_{2}\right)= \\
& N^{-}\left(\dot{\xi}_{1} ; \dot{\xi}_{2}\right) \overline{\mathcal{T}}^{s c-}\left(\dot{\xi}_{1} ; \dot{\xi}_{2}\right)+\mathrm{i}\left[N^{-}\left(-\dot{k}_{1}^{i n} ; \dot{\xi}_{2}\right)-N^{-}\left(\dot{\xi}_{1} ; \dot{\xi}_{2}\right)\right] \frac{\hat{\boldsymbol{\tau}}^{(\beta)}\left(\dot{\mathbf{k}}^{i n}\right)}{\dot{\xi}_{1}+\dot{k}_{1}^{i n}+i 0},
\end{aligned}
$$

so that the left-hand (right-hand) side of (91) is analytic in the upper (lower) half of the complex $\xi_{1}-$ plane. This means that both are one and the same entire function. It is easy to see that as $\dot{\xi}_{1} \rightarrow \infty$, this function is $O(1)$ and therefore, bounded. According to Liouville's Theorem, any such entire function is in fact a constant. Let us call it $\mathbf{g}$. Then multiplying both sides of $(91)$ first by $\left(M^{+}\right)^{-1}\left(\dot{\xi}_{1} ; \dot{\xi}_{2}\right)=N^{+}\left(\dot{\xi}_{1} ; \dot{\xi}_{2}\right)$ and then by $P^{-1}\left(\dot{\xi}_{1} ; \dot{\xi}_{2}\right)=P\left(\dot{\xi}_{1} ; \dot{\xi}_{2}\right) /\left(\xi_{1}^{2}+\xi_{2}^{2}\right)$ we obtain

$$
\begin{aligned}
\overline{\Delta U}_{i}^{s c}\left(\dot{\xi}_{1} ; \dot{\xi}_{2}\right)= & -\left[P^{-1}\left(\dot{\xi}_{1} ; \dot{\xi}_{2}\right) N^{+}\left(\dot{\xi}_{1} ; \dot{\xi}_{2}\right) \cdot\right. \\
& \left.\left(\frac{-\mathrm{i} N^{-}\left(-\dot{k}_{1}^{i n} ; \dot{\xi}_{2}\right) P\left(\dot{\xi}_{1} ; \dot{\xi}_{2}\right) \hat{\boldsymbol{\tau}}^{(\beta)}\left(\dot{\mathbf{k}}^{i n}\right)}{\dot{\xi}_{1}+\dot{k}_{1}^{i n}+\mathrm{i} 0}+\mathbf{g}\right)\right]_{i}, \\
\bar{T}_{i}^{s c-}\left(\dot{\xi}_{1} ; \dot{\xi}_{2}\right)_{i}= & {\left[P^{-1}\left(\dot{\xi}_{1} ; \dot{\xi}_{2}\right) M^{-}\left(\dot{\xi}_{1} ; \dot{\xi}_{2}\right) \cdot\right.} \\
& \left.\left(\frac{-\mathrm{i}\left[N^{-}\left(-\dot{k}_{1}^{i n} ; \dot{\xi}_{2}\right)-N^{-}\left(\dot{\xi}_{1} ; \dot{\xi}_{2}\right)\right] P\left(\dot{\xi}_{1} ; \dot{\xi}_{2}\right) \hat{\boldsymbol{\tau}}^{(\beta)}\left(\dot{\mathbf{k}}^{i n}\right)}{\dot{\xi}_{1}+\dot{k}_{1}^{i n}+i 0}+\mathbf{g}\right)\right]_{i} \quad i=1,2,
\end{aligned}
$$

where we have employed the formula (41). In order to find $\mathbf{g}$, we note that in view of (69), the plus function $\overline{\Delta \mathbf{U}}^{s c}\left(\dot{\xi}_{1} ; \dot{\xi}_{2}\right)$ has an apparent pole at $\dot{\xi}_{1}=\mathrm{i} \dot{\xi}_{2}$; similarly, the minus function $\overline{\mathbf{T}}^{s c-}\left(\dot{\xi}_{1} ; \dot{\xi}_{2}\right)$ has an apparent pole at $-i \dot{\xi}_{2}$. Since this is impossible, both corresponding residues must vanish. This gives us the following linear system for $\mathbf{g}$ :

$$
\begin{aligned}
& P\left(\mathrm{i} \dot{\xi}_{2} ; \dot{\xi}_{2}\right) N^{+}\left({ }_{\mathrm{i}} \dot{\xi}_{2} ; \dot{\xi}_{2}\right)\left(\frac{-\mathrm{i} N^{-}\left(-\dot{k}_{1}^{i n} ; \dot{\xi}_{2}\right) P\left(\mathrm{i} \dot{\xi}_{2} ; \dot{\xi}_{2}\right) \hat{\boldsymbol{\tau}}^{(\beta)}\left(\dot{\mathbf{k}}^{i n}\right)}{\mathrm{i} \dot{\xi}_{2}+\dot{k}_{1}^{i n}+\mathrm{i} 0}+\mathbf{g}\right)=\mathbf{0}, \\
& P\left(-\mathrm{i} \dot{\xi}_{2} ; \dot{\xi}_{2}\right) M^{-}\left(-\mathrm{i} \dot{\xi}_{2} ; \dot{\xi}_{2}\right) \cdot \\
& \left(\frac{-\mathrm{i}\left[N^{-}\left(-\dot{k}_{1}^{i n} ; \dot{\xi}_{2}\right)-N^{-}\left(-\mathrm{i} \dot{\xi}_{2} ; \dot{\xi}_{2}\right)\right] P\left(-\mathrm{i} \dot{\xi}_{2} ; \dot{\xi}_{2}\right) \hat{\boldsymbol{\tau}}^{(\beta)}\left(\dot{\mathbf{k}}^{i n}\right)}{-\mathrm{i} \dot{\xi}_{2}+\dot{k}_{1}^{i n}+i 0}+\mathbf{g}\right)=\mathbf{0} .
\end{aligned}
$$

Since $\left|P\left(\mathrm{i} \xi_{2} ; \xi_{2}\right)\right|=\left|P\left(-\mathrm{i} \xi_{2} ; \xi_{2}\right)\right|=0$, only two of the four equations above are linearly independent and thus, $\mathbf{g}$ is well defined. 
7. Solution of the 3D functional equation when the symmetry axis lies in the crack plane perpendicularly to the crack edge

7.1. The poles and branch points of $\hat{t}^{G}(\boldsymbol{\xi})$

When the symmetry axis lies in the crack plane perpendicularly to the crack edge we have $\phi_{0}=\theta_{0}=\pi / 2$,

$$
Q=\left[\begin{array}{ccc}
0 & 0 & 1 \\
-1 & 0 & 0 \\
0 & -1 & 0
\end{array}\right],
$$

and therefore,

$$
\xi_{1}=-\dot{\xi}_{2}, \xi_{2}=-\dot{\xi}_{3}, \xi_{3}=\dot{\xi}_{1} .
$$

The case of a normal incidence, $\dot{\xi}_{2} \equiv 0$, lends itself to an easy analytical treatment. Indeed, the poles of $\tilde{t}^{G}\left(\dot{\xi}_{1} ; 0\right)$, are respectively, the roots of the quartic equation

$$
\left(B_{1} \dot{\xi}_{1}^{2}+A_{11} \dot{\xi}_{3}^{2}-1\right)\left(A_{33} \dot{\xi}_{1}^{2}+B_{1} \dot{\xi}_{3}^{2}-1\right)-\left(A_{13}+B_{1}\right)^{2} \dot{\xi}_{1}^{2} \dot{\xi}_{3}^{2}=0
$$

and the quadratic equation

$$
B_{1} \dot{\xi}_{1}^{2}-1+B_{3} \xi_{3}^{2}=0,
$$

which correspond, respectively, to the first and second lines in (20), with the corresponding $\lambda^{(\alpha)}(\boldsymbol{\xi})=1$, both rewritten in terms of the crack coordinates. Moreover, Eq. (97) may be obtained from the first equation in (20) simply by putting $\dot{\xi}_{2}$ to zero and allowing $A_{11}$ and $A_{33}$ to exchange places. It follows that we can follow the form of solution presented in Section 6.1 and write

$$
\begin{aligned}
& \dot{\xi}_{3}^{(1)}\left(\dot{\xi}_{1} ; 0\right)=\dot{\xi}_{31}\left(\dot{\xi}_{1} ; 0\right)+\dot{\xi}_{32}\left(\dot{\xi}_{1} ; 0\right), \\
& \dot{\xi}_{3}^{(2)}\left(\dot{\xi}_{1} ; 0\right)=\dot{\xi}_{31}\left(\dot{\xi}_{1} ; 0\right)-\dot{\xi}_{32}\left(\dot{\xi}_{1} ; 0\right), \\
& \dot{\xi}_{3}^{(3)}\left(\dot{\xi}_{1} ; 0\right)=\left(B_{3}^{-1} B_{1}\right)^{1 / 2} \gamma_{2}\left(\dot{\xi}_{1} ; 0\right),
\end{aligned}
$$

with

$$
\begin{aligned}
& \dot{\xi}_{3 n}\left(\dot{\xi}_{1} ; 0\right)=\frac{\gamma_{6-3 n}}{2\left(B_{1} A_{11}\right)^{1 / 2}} \times \\
& \left\{\frac{\left[A_{13}^{2}+2 B_{1} A_{13}-A_{11} A_{33}\right] \xi_{1}^{2}+B_{1}+A_{11}-(-1)^{m} 2 B_{1}\left(A_{11} A_{33}\right)^{1 / 2} \gamma_{1} \gamma_{2}}{\gamma_{6-3 n}^{2}}\right\}^{1 / 2}, \\
& n=1,2,
\end{aligned}
$$

and

$$
\begin{aligned}
& \gamma_{0}=\gamma_{0}\left(\dot{\xi}_{1} ; 0\right)=\left(\kappa_{0}^{2}+\dot{\xi}_{1}^{2}\right)^{1 / 2}, \\
& \gamma_{\ell}=\gamma_{\ell}\left(\dot{\xi}_{1} ; 0\right)=\left(\kappa_{\ell}^{2}-\dot{\xi}_{1}^{2}\right)^{1 / 2}, \ell=1,2,3,
\end{aligned}
$$


with the branch points $\pm \mathrm{i} \kappa_{0}$ and $\pm \kappa_{\ell}, \ell=1,2,3$, where we have

$$
\kappa_{0}^{2}=C_{0}^{2}, \quad \kappa_{1}^{2}=A_{33}^{-1}, \quad \kappa_{2}^{2}=B_{1}^{-1}, \quad \kappa_{3}^{2}=C_{3}^{2}
$$

and

$$
\begin{aligned}
& C_{\ell}= \\
& D^{-1}\left\{2\left(A_{11} B_{1}\right)^{1 / 2}\left(B_{1}+A_{13}\right)\left[A_{13}^{2}-A_{11} A_{33}+B_{1}\left(A_{11}+2 A_{13}+A_{33}\right)\right]^{1 / 2}-\right. \\
& \left.(-1)^{\ell}\left[A_{13}\left(2 B_{1}+A_{13}\right)\left(B_{1}+A_{11}\right)+A_{11}\left(2 B_{1}^{2}+A_{33} B_{1}-A_{11} A_{33}\right)\right]\right\}^{1 / 2}, \\
& D=\left\{\left(A_{11} A_{33}-A_{13}^{2}\right)\left[\left(2 B_{1}+A_{13}\right)^{2}-A_{11} A_{33}\right]\right\}^{1 / 2} .
\end{aligned}
$$

The factorization described in Appendices A and B is applicable provided we have

$$
\kappa_{1}<\kappa_{2}<\kappa_{3} .
$$

This condition is satisfied by the austenitic steel under study.

\subsection{Decoupling the vector functional equations into the scalar functional equations}

In this case, $t_{i}^{\prime}=\sigma_{i 2}^{\prime}$ and the components of the transfer operator $S_{m}(\nabla)$ are given by

$$
\left(S_{m}\right)_{i k}(\nabla)=\Sigma_{i k}^{(2)}(\nabla) .
$$

Therefore, in view of (42), the transfer operator $S(\dot{\boldsymbol{\xi}})$ at $\dot{\xi}_{2}=0$ is

$$
S\left(\dot{\xi}_{1}, 0, \dot{\xi}_{3}\right)=\left[\begin{array}{ccc}
-B_{3} \dot{\xi}_{3} & 0 & 0 \\
0 & -A_{11} \dot{\xi}_{3} & A_{13} \dot{\xi}_{1} \\
0 & B_{1} \dot{\xi}_{1} & -B_{1} \dot{\xi}_{3}
\end{array}\right] .
$$

It is easy to check that if $\dot{\xi}_{2}=0$ and $A_{11}$ and $A_{33}$ exchange places the Fourier transform of the elastodynamic equation is the same as in the previous case. This means that $\widetilde{\tau}^{G}\left(\dot{\xi}_{1} ; 0\right)$ has the following diagonal form

$$
\widetilde{\tau}^{G}\left(\dot{\xi}_{1} ; 0\right)=\left[\begin{array}{ccc}
\mu_{1}\left(\dot{\xi}_{1} ; 0\right) & 0 & 0 \\
0 & \mu_{2}\left(\dot{\xi}_{1} ; 0\right) & 0 \\
0 & 0 & \mu_{3}\left(\dot{\xi}_{1} ; 0\right)
\end{array}\right],
$$

with the eigenvalues

$$
\begin{aligned}
& \mu_{1}\left(\dot{\xi}_{1} ; 0\right)=\frac{1}{2}\left(-B_{1} B_{3}\right)^{1 / 2} \gamma_{2}\left(\dot{\xi}_{1} ; 0\right), \\
& \mu_{2}\left(\dot{\xi}_{1} ; 0\right)=\left(\frac{A_{11}}{A_{33}}\right)^{1 / 2} \frac{\gamma_{2}\left(\dot{\xi}_{1} ; 0\right)}{\gamma_{1}\left(\dot{\xi}_{1} ; 0\right)} \mu_{3}\left(\dot{\xi}_{1} ; 0\right), \\
& \mu_{3}\left(\dot{\xi}_{1} ; 0\right)=\frac{\mathrm{i} R\left(\dot{\xi}_{1} ; 0\right)}{4 A_{11} \dot{\xi}_{31}\left(\dot{\xi}_{1} ; 0\right)},
\end{aligned}
$$


and the Rayleigh function

$$
R\left(\dot{\xi}_{1} ; 0\right)=\left(A_{13}^{2}-A_{11} A_{33}\right) \dot{\xi}_{1}^{2}+A_{11}+\left(A_{11} A_{33}\right)^{1 / 2} \frac{\gamma_{1}\left(\dot{\xi}_{1} ; 0\right)}{\gamma_{2}\left(\dot{\xi}_{1} ; 0\right)} .
$$

Substituting (107) into the 3D vector functional equation (57), the latter decouples into three scalar equations,

$$
\mu_{i}\left(\dot{\xi}_{1} ; 0\right) \overline{\Delta U}_{i}^{s c}\left(\dot{\xi}_{1} ; 0\right)=\mathrm{i} \frac{\widehat{t}_{i}^{(\beta)}\left(\dot{k}_{1}^{i n}, \dot{k}_{2}^{i n}, 0\right)}{\dot{\xi}_{1}+\dot{k}_{1}^{i n}+i 0}-\bar{T}_{i}^{s c-}\left(\dot{\xi}_{1} ; 0\right), i=1,2,3 .
$$

The equations have the same structure as (73) and therefore, can all be solved using the Wiener-Hopf technique by introducing the factorization

$$
\mu_{i}\left(\dot{\xi}_{1} ; 0\right)=\mu_{i}^{+}\left(\dot{\xi}_{1} ; 0\right) \mu_{i}^{-}\left(\dot{\xi}_{1} ; 0\right), i=1,2,3 .
$$

On allowing $A_{11}$ and $A_{33}$ to exchange places, the tangential traction $\mu_{3}\left(\dot{\xi}_{1} ; 0\right)$ is the same as the tangential traction $\mu_{1}\left(\dot{\xi}_{1} ; 0\right)$ in Section 6 . Therefore, it can be factored in the same manner, with $l_{0}, l_{1}, K_{0}$ and $K_{1}$ the same as in the Appendices A and B but with $A_{11}$ standing in place of $A_{33}$ and vice versa.

It follows that the considerations and notations introduced in Appendices A and B allow us to define $\mu_{i}^{ \pm}\left(\dot{\xi}_{1} ; 0\right)$ as

$$
\begin{aligned}
& \mu_{1}^{ \pm}\left(\dot{\xi}_{1} ; 0\right)=\left(-\frac{B_{1} B_{3}}{4}\right)^{1 / 4} \gamma_{2}^{ \pm}\left(\dot{\xi}_{1} ; 0\right), \\
& \mu_{2}^{ \pm}\left(\dot{\xi}_{1} ; 0\right)=\left(\frac{A_{11}}{A_{33}}\right)^{1 / 4} \frac{\gamma_{2}^{ \pm}\left(\dot{\xi}_{1} ; 0\right)}{\gamma_{1}^{ \pm}\left(\dot{\xi}_{1} ; 0\right)} \mu_{3}^{ \pm}\left(\dot{\xi}_{1} ; 0\right), \\
& \mu_{3}^{ \pm}\left(\dot{\xi}_{1} ; 0\right)=\left(-\frac{l_{0}}{4 l_{1} A_{11}}\right)^{1 / 2} \frac{K_{0}^{ \pm}\left(\dot{\xi}_{1} ; 0\right)}{\gamma_{3}^{ \pm}\left(\dot{\xi}_{1} ; 0\right) K_{1}^{ \pm}\left(\dot{\xi}_{1} ; 0\right)}\left(\kappa_{R} \pm \dot{\xi}_{1}\right),
\end{aligned}
$$

where, similarly to (80), we have

$$
\gamma_{\ell}^{ \pm}\left(\dot{\xi}_{1} ; 0\right)=\left(\kappa_{\ell} \pm \dot{\xi}_{1}\right)^{1 / 2}, \ell=1,2,3 .
$$

It is easy to see from their definition that the $+(-)$ functions above have no zeros in the upper (lower) half of the complex $\dot{\xi}_{1}-$ plane. It follows that we have

$$
\overline{\Delta U}_{i}^{s c}\left(\dot{\xi}_{1} ; 0\right)=\mathrm{i} \frac{\widehat{t}_{i}^{(\beta)}\left(\dot{\mathbf{k}}^{i n}\right)}{\left(\dot{\xi}_{1}+\dot{k}_{1}^{i n}+i 0\right) \mu_{i}^{+}\left(\dot{\xi}_{1} ; 0\right) \mu_{i}^{-}\left(-\dot{k}_{1}^{i n} ; 0\right)}, i=1,2,3 .
$$

\section{Diffraction coefficients}

Let us consider the equation

$$
\mathbf{U}^{s c}\left(\dot{x}_{1}, \dot{x}_{3} ; \dot{\xi}_{2}\right)=-\frac{1}{2 \pi} \int_{-\infty}^{\infty} \widetilde{t}^{G}\left(\dot{\xi}_{1}, \dot{x}_{3} ; \dot{\xi}_{2}\right) \overline{\Delta \mathbf{U}}^{s c}\left(\dot{\xi}_{1} ; \dot{\xi}_{2}\right) \mathrm{e}^{-\mathrm{i} \dot{\xi}_{1} \dot{x}_{1}} d \dot{\xi}_{1}
$$

which follows from Eq. (48) via the inverse Fourier transform in $\dot{\xi}_{1}$. Substituting (51) into (115) leads us to 


$$
\begin{aligned}
& \mathbf{U}^{s c}\left(\dot{x}_{1}, \dot{x}_{3} ; \dot{\xi}_{2}\right)= \pm \frac{\mathrm{i}}{2 \pi} .
\end{aligned}
$$

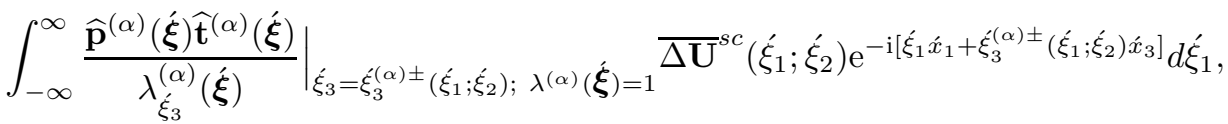

where summation over $\alpha$ is implied and the top (bottom) sign is chosen when $\dot{x}_{3} \geq 0+$ $\left(\dot{x}_{3} \leq 0-\right)$.

Let us now introduce the polar coordinates $\dot{r}_{\perp}$ and $\dot{\theta}$ such that we have

$$
\begin{aligned}
& \dot{x}_{1}=\dot{r}_{\perp} \cos \dot{\theta}, \\
& \dot{x}_{3}=\dot{r}_{\perp} \sin \dot{\theta}, \dot{\theta} \in[0,2 \pi] .
\end{aligned}
$$

When values of $\dot{k}^{i n} \dot{r}_{\perp}$ are large, the main contributions to (116) come from the stationary phase points and other critical points that are described e.g. in [8]. The method of the uniform stationary phase (see e.g. [17], [16]) allows us to treat the situations when the critical points coalesce, that is when an observation point lies in a transition zone between geometrical regions, but here we treat only those observation points for which the critical points are isolated, that is the observation points that lie in geometrical zones. Thus, the formulas given below are not applicable near the shadow boundaries, cuspidal edges and conical points of the $q S V$ wave surface or points of tangential contact of the $q S H$ and $q S V$ wave surfaces (see e.g. [8] and [20]) The contribution of each isolated phase stationary point can be evaluated using the standard stationary phase formula,

$$
\int_{-\infty}^{\infty} g(t) e^{i r f(t)} d t \sim g(c)\left[\frac{2 \pi \mathrm{i}}{r \ddot{f}(c)}\right]^{1 / 2} \mathrm{e}^{\mathrm{i} r f(c)},
$$

where the dot denotes the derivative with respect to the argument (see e.g. [18]); and $c$ is such that $\dot{f}(c)=0$. In both symmetric cases considered above, the phase function in (116) can be written as

$$
-\dot{\xi}_{1} \dot{x}_{1}+\dot{\xi}_{3}^{(\alpha)}\left(\dot{\xi}_{1} ; \dot{\xi}_{2}\right)\left|\dot{x}_{3}\right|=-\dot{r}_{\perp}\left[\dot{\xi}_{1} \cos \dot{\theta}-\dot{\xi}_{3}^{(\alpha)}\left(\dot{\xi}_{1} ; \dot{\xi}_{2}\right)|\sin \hat{\theta}|\right] .
$$

Let us call each solution $\dot{\xi}_{1}$ of the equation

$$
-\cos \dot{\theta}+\dot{\xi}_{3}^{(\alpha)}\left(\dot{\xi}_{1} ; \dot{\xi}_{2}\right)|\sin \dot{\theta}|=0
$$

the stationary phase point $\dot{\xi}_{1}^{(\alpha)}$. Then applying (118) to (116) and multiplying both sides of the resulting formula by $\exp \left(\mathrm{i} \hat{k}_{2}^{i n} \dot{x}_{2}\right)$ the main contributions to the scattered field in the geometrical zones are given by the stationary points $\dot{\xi}_{1}^{(\alpha)}$, so that for each fixed $\alpha$ and $\beta$, we have the GTD approximation

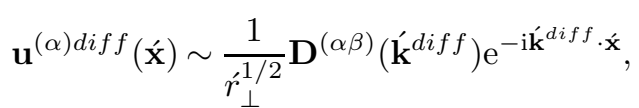

where using (45), the dimensionless diffracted wave vector is

$$
\dot{\mathbf{k}}^{d i f f}=\left(\dot{\xi}_{1}^{(\alpha)},-\dot{k}_{2}^{i n},-\operatorname{sgn}(\sin \dot{\theta}) \dot{\xi}_{3}^{(\alpha)}\left(\dot{\xi}_{1}^{(\alpha)} ;-\dot{k}_{2}^{i n}\right)\right) ;
$$


and the vector diffraction coefficients $\mathbf{D}^{(\alpha \beta)}$ are such that we can write

$$
\begin{aligned}
\mathbf{D}^{(\alpha \beta)}\left(\dot{\mathbf{k}}^{\text {diff }}\right)= & -\operatorname{sgn}(\sin \dot{\theta}) \frac{1}{\left[2 \pi \mathrm{i}|\sin \theta \dot{\xi}| \ddot{\xi}_{3}^{(\alpha)}\left(\dot{k}_{1}^{\text {diff }} ;-\dot{k}_{2}^{i n}\right)\right]^{1 / 2}} . \\
& \left.\frac{\widehat{\mathbf{p}}^{(\alpha)}\left(\dot{\mathbf{k}}^{\text {diff }}\right) \widehat{\mathbf{t}}^{(\alpha)}\left(\dot{\mathbf{k}}^{\text {diff }}\right)}{\lambda_{\dot{\xi}_{3}}^{(\alpha)}\left(\hat{\mathbf{k}}^{d i f f}\right)}\right|_{\lambda^{(\alpha)}\left(\dot{\mathbf{k}}^{\text {diff }}\right)=1} \widehat{\Delta \mathbf{U}}^{s c}\left(\dot{k}_{1}^{\text {diff }} ;-\dot{k}_{2}^{\text {in }}\right),
\end{aligned}
$$

with no summation over $\alpha$. We remind the reader that the first superscript $\alpha=1,2$ or 3 describes a diffracted wave and the second superscript $\beta=1,2$ or 3 , an incident wave. In the case of the symmetry axis perpendicular to the crack plane we have

$$
\begin{aligned}
& \lambda_{\dot{\xi}_{3}}^{(\alpha)}(\dot{\boldsymbol{\xi}})=-2 \dot{\xi}_{3} \frac{\left(A_{13}^{2}+2 B_{1} A_{13}-A_{11} A_{33}\right) \dot{\xi}_{\perp}^{2}-2 B_{1} A_{33} \dot{\xi}_{3}^{2}+\left(B_{1}+A_{33}\right) \lambda^{(\alpha)}(\dot{\boldsymbol{\xi}})}{\left(B_{1}+A_{11}\right) \dot{\xi}_{\perp}^{2}+\left(B_{1}+A_{33}\right) \dot{\xi}_{3}^{2}-2 \lambda^{(\alpha)}(\dot{\boldsymbol{\xi}})} \quad, \quad \alpha=1,2, \\
& \lambda_{\xi_{3}}^{(3)}(\dot{\boldsymbol{\xi}})=2 B_{1} \dot{\xi}_{3},
\end{aligned}
$$

where when $\left|\dot{\xi}_{3}\right|=\left|\dot{\xi}_{3}^{(\alpha)}\left(\dot{\xi}_{1} ;-\dot{k}_{2}^{i n}\right)\right|, \lambda^{(\alpha)}(\dot{\boldsymbol{\xi}})=1$. In the case of the symmetry axis lying in the crack plane perpendicularly to the crack edge the above formulas apply for $\xi_{2}=0$ if $A_{11}$ and $A_{33}$ exchange places, so that we have

$$
\begin{aligned}
& \lambda_{\dot{\xi}_{3}}^{(\alpha)}(\dot{\boldsymbol{\xi}})=-2 \dot{\xi}_{3} \frac{\left(A_{13}^{2}+2 B_{1} A_{13}-A_{11} A_{33}\right) \dot{\xi}_{1}^{2}-2 B_{1} A_{11} \dot{\xi}_{3}^{2}+\left(B_{1}+A_{11}\right) \lambda^{(\alpha)}(\dot{\boldsymbol{\xi}})}{\left(B_{1}+A_{33}\right) \dot{\xi}_{1}^{2}+\left(B_{1}+A_{11}\right) \dot{\xi}_{3}^{2}-2 \lambda^{(\alpha)}(\dot{\boldsymbol{\xi}})}, \quad, \quad \alpha=1,2, \\
& \lambda_{\dot{\xi}_{3}}^{(3)}(\dot{\boldsymbol{\xi}})=2 B_{3} \dot{\xi}_{3} .
\end{aligned}
$$

Let us now introduce the spherical polar angles $\dot{\varphi}$ and $\hat{\vartheta}$ associated with the crack coordinate system. Then each incident unit wave vector can be expressed in terms of its medium coordinates as

$$
\mathbf{n}^{i n}=\left(\sin \dot{\varphi}^{i n} \cos \hat{\vartheta}^{i n}, \cos \varphi^{i n}, \sin \varphi^{i n} \sin \vartheta^{i n}\right) .
$$

When the symmetry axis lies in the crack plane and is perpendicular to the crack edge we only consider the incident vectors that are perpendicular to the crack edge, i.e. we assume $\dot{\varphi}^{\text {in }}=90^{\circ}$. Using the transpose of (95), this yields the following medium coordinates of the incident unit wave vector $\mathbf{n}^{i n}$ :

$$
\mathbf{n}^{i n}=\left(0,-\sin \vartheta^{i n}, \cos \vartheta^{i n}\right) .
$$

The incident wave number $k^{(\beta)}$ and parameter $k_{2}^{i n}$ can be found by using the formulae (37) and (45), respectively. In each case, the polarization of the incident wave $\widehat{\mathbf{p}}^{(\beta)}\left(\hat{\mathbf{k}}^{\text {in }}\right)$ is given by (22) and (36).

Thus, each incident wave vector of type $\beta=1,2$ or 3 produces three families of diffracted wave vectors of type $\alpha=1,2$ or 3 , each covering a portion of the cone surface, $q P, q S V$ or $q S H$, respectively, with each cone having a cross-section in the shape of the respective slowness surface (see Fig. 3 b). Each wave vector can be expressed as 


$$
\dot{\mathbf{k}}^{\text {diff }}=-\left(\varrho^{(\alpha)}\left(\dot{\vartheta} ;-\hat{k}_{2}^{i n}\right) \cos \dot{\vartheta}, \dot{k}_{2}^{i n}, \varrho^{(\alpha)}\left(\dot{\vartheta} ;-\hat{k}_{2}^{i n}\right) \sin \dot{\vartheta}\right), \quad \dot{\vartheta} \in[0,2 \pi],
$$

where in the case of the symmetry axis perpendicular to the crack plane we have

$$
\begin{aligned}
& \varrho^{(1)}\left(\dot{\vartheta} ;-\dot{k}_{2}^{i n}\right)=\left[\frac{-D_{2}+\left(D_{2}^{2}-4 D_{1} D_{3}\right)^{1 / 2}}{2 D_{1}}\right]^{1 / 2}, \\
& \varrho^{(2)}\left(\dot{\vartheta} ;-\dot{k}_{2}^{i n}\right)=\left[\frac{-D_{2}-\left(D_{2}^{2}-4 D_{1} D_{3}\right)^{1 / 2}}{2 D_{1}}\right]^{1 / 2}, \\
& \varrho^{(3)}\left(\dot{\vartheta} ;-\dot{k}_{2}^{i n}\right)=\left[\frac{1-B_{3}\left(\hat{k}_{2}^{i n}\right)^{2}}{B_{3} \cos \hat{\vartheta}^{2}+B_{1} \sin \hat{\vartheta}^{2}}\right]^{1 / 2},
\end{aligned}
$$

with

$$
\begin{aligned}
& D_{1}=B_{1} A_{33} \sin ^{4} \hat{\vartheta}-B_{4} \cos ^{2} \hat{\vartheta} \sin ^{2} \hat{\vartheta}+A_{11} B_{1} \cos ^{4} \hat{\vartheta} \\
& D_{2}=\left(2 A_{11}\left[k_{2}^{i n}\right]^{2} B_{1}-B_{1}-A_{11}\right) \cos ^{2} \dot{\vartheta}-\left(B_{4}\left[\hat{k}_{2}^{i n}\right]^{2}+A_{33}+B_{1}\right) \sin ^{2} \hat{\vartheta}, \\
& D_{3}=\left(A_{11}\left[k_{2}^{i n}\right]^{2}-1\right)\left(B_{1}\left[\hat{k}_{2}^{i n}\right]^{2}-1\right), \quad B_{4}=A_{13}^{2}+2 A_{13} B_{1}-A_{11} A_{33},
\end{aligned}
$$

and in the case of the symmetry axis lying in the crack plane perpendicularly to the crack edge we can write

$$
\begin{aligned}
& \varrho^{(1)}(\dot{\vartheta} ; 0)=\left[\frac{-D_{2}+\left(D_{2}^{2}-4 D_{1}\right)^{1 / 2}}{2 D_{1}}\right]^{1 / 2}, \\
& \varrho^{(2)}(\dot{\vartheta} ; 0)=\left[\frac{-D_{2}-\left(D_{2}^{2}-4 D_{1}\right)^{1 / 2}}{2 D_{1}}\right]^{1 / 2}, \\
& \varrho^{(3)}(\dot{\vartheta} ; 0)=\left[\frac{1}{B_{1} \cos ^{2} \hat{\vartheta}+B_{3} \sin ^{2} \hat{\vartheta}}\right]^{1 / 2} .
\end{aligned}
$$

with

$$
\begin{aligned}
& D_{1}=B_{1} A_{11} \sin ^{4} \dot{\vartheta}-B_{4} \cos ^{2} \dot{\vartheta} \sin ^{2} \dot{\vartheta}+A_{33} B_{1} \cos ^{4} \dot{\vartheta} \\
& D_{2}=-\left(B_{1}+A_{33}\right) \cos ^{2} \dot{\vartheta}-\left(A_{11}+B_{1}\right) \sin ^{2} \dot{\vartheta} .
\end{aligned}
$$

Using the fact that the wave front is a polar reciprocal of the slowness surface, it can be shown that in all cases, the polar angles $\dot{\theta}$ and $\hat{\vartheta}$ of the ray and wave vector, respectively, are related by the following formula

$$
\dot{\theta}=\dot{\vartheta}-\tan ^{-1}\left(\frac{1}{\varrho} \frac{\partial \varrho}{\partial \hat{\vartheta}}\right) \text {. }
$$

Similarly to [19], in Appendices C and D we plot for various incident polar angles $\varphi^{\text {in }}$ and $\vartheta^{i n}$, the magnitudes of the diffracted coefficients $D^{\gamma, \delta}$, where $\gamma, \delta=q P, q S V$ or $q S H$ versus the diffracted polar angle $\dot{\vartheta} \in[0,2 \pi]$, thus tracing their variation over the corresponding slowness curves. As expected, all the graphs for the magnitudes $D^{(q S H, q S H)}$ are similar to the respective graphs for the magnitudes $D^{S H, S H}$ given in [19]. Two representative graphs of the magnitudes of the diffracted coefficients $D^{(q P, q P)}$ versus the physical 


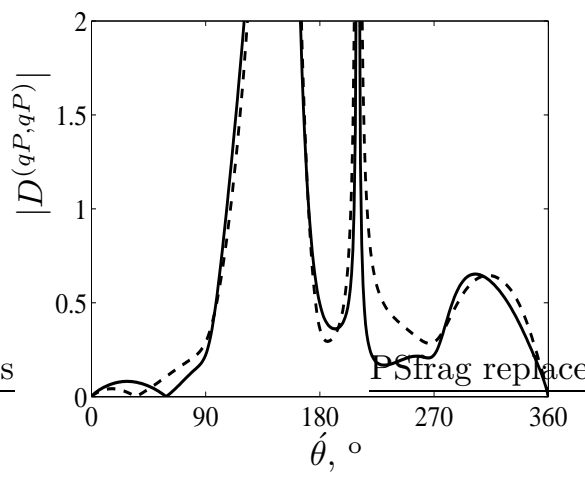

b

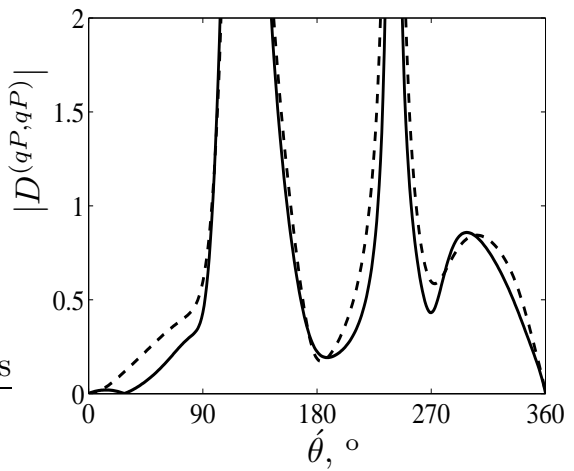

Fig. 4. The magnitude of the diffraction coefficient $D^{(q P, q P)}$ versus the physical polar angle $\theta$ for the symmetry axis perpendicular to the crack plane (solid line) and the symmetry axis lying in the crack plane perpendicularly to the crack edge (dashed line). The incident angles are $\phi^{i n}=90^{\circ}$ and $(\mathrm{a}) \dot{\theta}^{i n}=120^{\circ}$, (b) $\theta^{i n}=150^{\circ}$.

polar angle $\dot{\theta} \in[0,2 \pi]$, that is tracing their variation over the corresponding wave curves are given in Fig. 4.

Finally, we note that the GTD approximation (121) breaks down in the so-called transition regions between various geometrical zones when the denominator in (123) is small or vanishes. The regions center on the light-shadow boundaries, cuspidal edges and conical points where, respectively, $\dot{k}_{1}^{\text {diff }}+\dot{k}_{1}^{i n}, \ddot{\xi}_{3}^{(\alpha)}\left(\dot{k}_{1}^{\text {diff }} ;-\dot{k}_{2}^{i n}\right)$ and $\lambda_{\dot{\xi}_{3}}^{(\alpha)}\left(\dot{\mathbf{k}}^{\text {diff }}\right)$ turn to zero. When the singularities are few and far between, like in the isotropic case considered in [19] or in the cases presented in our Appendices C and D, there is no practical need for developing the Uniform GTD that provides a good approximation of the diffracted field in transition zones as well as the geometrical ones. However, when cuspidal edges and conical points are present the formula (123) ceases to be of much practical use and the Uniform GTD description should be used instead of (121) (see e.g. [8]). We illustrate the situation in Appendix E but do not plot the corresponding "diffraction coefficients" in Appendices C and D.

\section{Conclusions}

We have considered a semi-infinite crack embedded in a transversely isotropic medium and studied two special cases, one, in which the axis of symmetry is normal to the crack face and the wave incidence is arbitrary and another, in which the axis lies in the crack plane normal to the edge and the incident wave vector is also normal to the edge. The problem is of interest in NDE, because austenitic steels that are found in claddings and other welds in the nuclear reactors can often be modeled as transversely isotropic. In both cases, we have expressed the scattered field in a closed form and computed the corresponding diffraction coefficients.

A simple case of normal incidence in a TI material that supports three convex slowness surfaces has been considered before [7]. This article and its published version [21] addresses an extra complication which arises when a slowness surface contains inflections 
and in the first of the above cases, the incidence is allowed to be oblique. In future, we plan to cross-validate our code with other numerical codes, such as the one reported in [13], and also validate it against experimental data - whenever the latter become available.

\section{Acknowledgement}

Partial support of this work has been provided by CEA LIST under the CIVA 2012 Carnot project. We are grateful to Professor Borovikov for many useful comments.

\section{References}

[1] J. D. Achenbach, A.K. Gautesen and H. McMaken, Ray Methods for Waves in Elastic Solids; With Applications to Scattering by Cracks, Pitman, N.Y. (1982).

$[2]$ G. F. D. Duff, "The Cauchy problem for elastic waves in an anisotropic medium" Phil. Trans. R. Soc. Lond. 252, 249-273 (1960).

[3] R. G. Payton, Elastic Wave Propagation in Transversely Isotropic Media, Martinus Nijhoff, The Hague (1983).

[4] J. B. Keller, "Diffraction by an Aperture" J. Appl. Phys. 28(4), 426-444 (1957).

[5] J. B. Keller, "A geometrical theory of diffraction" Calculus of Variations and Its Applications 8 (1958).

[6] J. B. Keller, "Rays, waves and asymptotics" Bull. Am. Math. Soc. 87 (1978).

[7] A. N. Norris and J. D. Achenbach, "Elastic wave diffraction by a semi- infinite crack in a transversely isotropic medium" Quart. J. Mech. Appl. Math. 37, 565-580 (1984).

[8] D. Gridin, "Far-field asymptotics of the Green's tensor for a transversely isotropic solid" Proceedings of the Royal Society of London A456, 571-591 (2000).

[9] J. G. Harris, Linear Elastic Waves, Cambridge University Press, Cambridge (2001).

[10] S. N. Karp and F. C. Karal, "The Elastic-Field Behaviour in the Neighborhood of a Crack of Arbitrary Angle" Comm. Pure and Appl. Math. 15, 413-421 (1962a).

[11] S. N. Karp and F. C. Karal, "Stress behaviour in the neighborhood of sharp corners" Geophys. 29, 360-369 (1962b).

[12] J. P. Charlesworth and J. A. G. Temple, Engineering Applications of Ultrasonic Time-Of-Flight Diffraction, Research Studies Press, Sommerset (1989).

[13] P. A. Lewis, J. A. G. Temple, E. J. Walker and G. R. Wickham, "Calculation of diffraction coefficients for a semi-infinite crack embedded in an infinite anisotropic linearly elastic body" Proc. R. Soc. Lond. 454, 1781-1803 (1996).

[14] P. A. Lewis, Factorization of a certain Wiener-Hopf matrix arising in diffraction theory. In "Diffraction and Scattering in Fluid Mechanics and Elasticity" (2000).

[15] B. Noble, Methods Based on the Wiener-Hopf Technique for the Solution of Partial Differential Equations, Pergamon Press, Oxford (1960).

[16] N. Bleistein and R. A. Handelsman Asymptotic Expansions of Integrals, Dover Publications Inc., N. Y. (1986).

[17] V. A. Borovikov, Uniform Stationary Phase Method, IEE, London (1994).

[18] R. Wong, Asymptotic Approximations of Integrals, Academic Press, London (1989).

[19] J. D. Achenbach and A. K. Gautesen, Edge Diffraction in Acoustics and Elastodynamics. In "Low and High Frequency Asymptotics", Vol. 2, Eds. V.K. Varadan and V.V. Varadan (Elsevier Science Publishers, Amsterdam), pp. 335-401 (1986).

[20] D. Gridin and L. Ju. Fradkin, "Far-field asymptotic description of a point source acting on a transversely isotropic half-space" Proceedings of the Royal Society of London A457, 2675-2698 (2001).

[21] A. Gautesen, V. Zernov and L. Fradkin, "Diffraction coefficients of a semi-infinite planar crack embedded in a transversely isotropic space", Wave Motion, 46(10), 29-46, (2009). 


\section{Appendix A. Factorization of the numerator of $\mu_{1}\left(\dot{\xi}_{1} ;-\dot{k}_{2}^{i n}\right)$}

In order to factorize the numerator we introduce

$$
K_{0}\left(\dot{\xi}_{1} ; \dot{\xi}_{2}\right)=K_{0}^{*}\left(\dot{\xi}_{1}, \frac{\gamma_{1}\left(\dot{\xi}_{1} ; \dot{\xi}_{2}\right)}{\gamma_{2}\left(\dot{\xi}_{1} ; \dot{\xi}_{2}\right)}\right)=\frac{R\left(\dot{\xi}_{1} ; \dot{\xi}_{2}\right)}{l_{0}\left(\kappa_{R}^{2}-\dot{\xi}_{1}^{2}\right)}
$$

where $\kappa_{R}=\left(k_{R}^{2}-\xi_{2}^{2}\right)^{1 / 2}$ and

$$
l_{0}=\lim _{\xi_{1} \rightarrow \infty} \frac{R\left(\dot{\xi}_{1} ; \dot{\xi}_{2}\right)}{\kappa_{R}^{2}-\dot{\xi}_{1}^{2}}=A_{11} A_{33}-A_{13}^{2} .
$$

The function $K_{0}\left(\dot{\xi}_{1} ; \dot{\xi}_{2}\right)$ thus defined tends to unity at infinity, is never zero and involves the ratio $\gamma_{1} / \gamma_{2}$. The branch cuts of $R\left(\dot{\xi}_{1} ; \dot{\xi}_{2}\right)$ and therefore $K_{0}\left(\dot{\xi}_{1} ; \dot{\xi}_{2}\right)$ that lie in the lower half plane are presented in Fig. A.1.

a

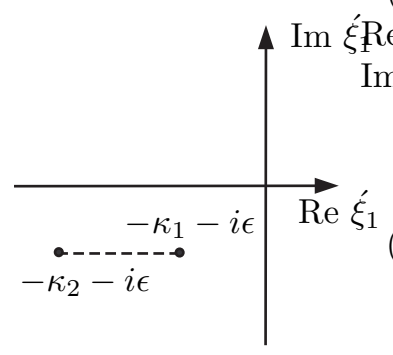

b

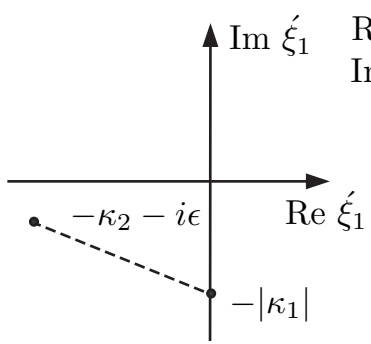

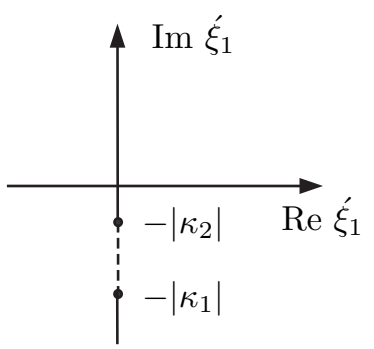

Fig. A.1. The branch cuts of function $K_{0}\left(\xi_{1} ; \xi_{2}\right)$ for a) $\kappa_{1}$ imaginary and $\kappa_{2}$ real; b) $\kappa_{1}$ and $\kappa_{2}$ real; c) $\kappa_{1}$ and $\kappa_{2}$ imaginary.

Closing the integration contour in the lower half-space, we can write

$$
\begin{aligned}
& \ln K_{0}^{+}\left(\dot{\xi}_{1} ; \dot{\xi}_{2}\right)=\frac{1}{2 \pi \mathrm{i}} \int_{-\infty}^{\infty} \frac{\ln K_{0}\left(t ; \dot{\xi}_{2}\right)}{t-\dot{\xi}_{1}} d t= \\
& \frac{1}{2 \pi \mathrm{i}} \int_{-\kappa_{2}}^{-\kappa_{1}} \frac{\Delta \ln K_{0}\left(t ; \dot{\xi}_{2}\right)}{t-\dot{\xi}_{1}} d t
\end{aligned}
$$

where $\ln K_{0}^{+}\left(\dot{\xi}_{1} ; \dot{\xi}_{2}\right)$ is analytic in the upper half of the $\dot{\xi}_{1}$ plane, the jump over the cut is

$$
\Delta \ln K_{0}\left(t ; \dot{\xi}_{2}\right)=\ln K_{0}^{*}\left(t, \frac{\gamma_{1}\left(t ; \dot{\xi}_{2}\right)}{\gamma_{2}\left(t ; \dot{\xi}_{2}\right)}\right)-\ln K_{0}^{*}\left(t,-\frac{\gamma_{1}\left(t ; \dot{\xi}_{2}\right)}{\gamma_{2}\left(t ; \dot{\xi}_{2}\right)}\right)
$$

and we have

$$
K_{0}\left(\dot{\xi}_{1} ; \dot{\xi}_{2}\right)=K_{0}^{+}\left(\dot{\xi}_{1} ; \dot{\xi}_{2}\right) K_{0}^{-}\left(\dot{\xi}_{1} ; \dot{\xi}_{2}\right),
$$

with 


$$
K_{0}^{-}\left(\dot{\xi}_{1} ; \dot{\xi}_{2}\right)=K_{0}^{+}\left(-\dot{\xi}_{1} ; \dot{\xi}_{2}\right)
$$

analytic in the lower half of the $\dot{\xi}_{1}$-plane. By changing $t$ to $-t$, the formula (A.3) can be simplified to

$$
\ln K_{0}^{+}\left(\dot{\xi}_{1} ; \dot{\xi}_{2}\right)=-\frac{1}{2 \pi \mathrm{i}} \int_{\kappa_{1}}^{\kappa_{2}} \frac{\Delta \ln K_{0}\left(t ; \dot{\xi}_{2}\right)}{t+\dot{\xi}_{1}} d t
$$

When $-\dot{\xi}_{1}$ lies on the branch cut $\left[\kappa_{2}, \kappa_{1}\right]$, integration of the singular integrand can be avoided by using the formula

$$
K_{0}^{+}\left(\dot{\xi}_{1} ; \dot{\xi}_{2}\right)=\frac{1}{K_{0}^{+}\left(-\dot{\xi}_{1} ; \dot{\xi}_{2}\right)} K_{0}\left(\dot{\xi}_{1} ; \dot{\xi}_{2}\right) .
$$

\section{Appendix B. Factorization of the denominator of $\mu_{1}\left(\xi_{1} ; \xi_{2}\right)$}

In order to factorize the denominator of $\mu_{1}\left(\xi_{1}\right)$ we introduce the function

$$
K_{1}\left(\dot{\xi}_{1} ; \dot{\xi}_{2}\right)=K_{1}^{*}\left(\dot{\xi}_{1}, \frac{\gamma_{1}\left(\dot{\xi}_{1} ; \dot{\xi}_{2}\right)}{\gamma_{2}\left(\dot{\xi}_{1} ; \dot{\xi}_{2}\right)}\right)=\frac{\dot{\xi}_{31}\left(\dot{\xi}_{1} ; \dot{\xi}_{2}\right)}{l_{1} \gamma_{3}\left(\dot{\xi}_{1} ; \dot{\xi}_{2}\right)},
$$

where we have

$$
l_{1}=\lim _{\xi_{1} \rightarrow \infty} \frac{\dot{\xi}_{31}\left(\dot{\xi}_{1} ; \dot{\xi}_{2}\right)}{\gamma_{3}\left(\dot{\xi}_{1} ; \dot{\xi}_{2}\right)}=\left[\frac{2 B_{1}\left(A_{11} A_{33}\right)^{1 / 2}-A_{13}^{2}-2 B_{1} A_{13}+A_{11} A_{33}}{4 B_{1} A_{33}}\right]^{1 / 2} .
$$

The function tends to unity at infinity, is never zero and involves the ratio $\gamma_{1} / \gamma_{2}$. Using the same considerations as above, leads us to

$$
\ln K_{1}^{+}\left(\dot{\xi}_{1} ; \dot{\xi}_{2}\right)=-\frac{1}{2 \pi \mathrm{i}} \int_{\kappa_{1}}^{\kappa_{2}} \frac{\Delta \ln K_{1}\left(t ; \dot{\xi}_{2}\right)}{t+\dot{\xi}_{1}} d t
$$

where the jump over the cut is

$$
\Delta \ln K_{1}\left(t ; \dot{\xi}_{2}\right)=\ln K_{1}^{*}\left(t, \frac{\gamma_{1}\left(t ; \dot{\xi}_{2}\right)}{\gamma_{2}\left(t ; \dot{\xi}_{2}\right)}\right)-\ln K_{1}^{*}\left(t,-\frac{\gamma_{1}\left(t ; \dot{\xi}_{2}\right)}{\gamma_{2}\left(t ; \dot{\xi}_{2}\right)}\right) .
$$

We note that

$$
K_{1}\left(\dot{\xi}_{1} ; \dot{\xi}_{2}\right)=K_{1}^{+}\left(-\dot{\xi}_{1} ; \dot{\xi}_{2}\right) K_{1}^{-}\left(\dot{\xi}_{1} ; \dot{\xi}_{2}\right)
$$

with the function

$$
K_{1}^{-}\left(\dot{\xi}_{1} ; \dot{\xi}_{2}\right)=K_{1}^{+}\left(-\dot{\xi}_{1} ; \dot{\xi}_{2}\right)
$$

analytic in the lower half of the $\dot{\xi}_{1}-$ plane. It follows that when $-\dot{\xi}_{1}$ lies on the branch cut $\left[\kappa_{2}, \kappa_{1}\right]$, integration of the singular integrand can be avoided by using the formula

$$
K_{1}^{+}\left(\dot{\xi}_{1} ; \dot{\xi}_{2}\right)=\frac{1}{K_{1}^{+}\left(-\dot{\xi}_{1} ; \dot{\xi}_{2}\right)} K_{1}\left(\dot{\xi}_{1} ; \dot{\xi}_{2}\right) .
$$


Appendix C. Magnitudes of various diffraction coefficients for the axis of symmetry perpendicular to the crack

In Fig. C.4 below, when the mode of the incident wave is $q S V$ and the corresponding diffracted wave vectors are complex, we artificially set the diffraction coefficients to zero.

a

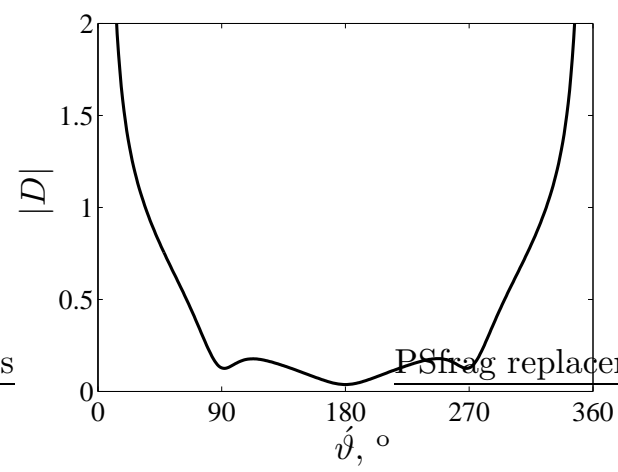

b

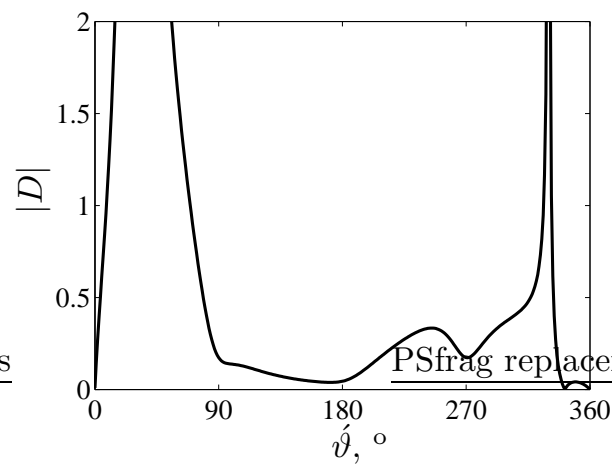

c

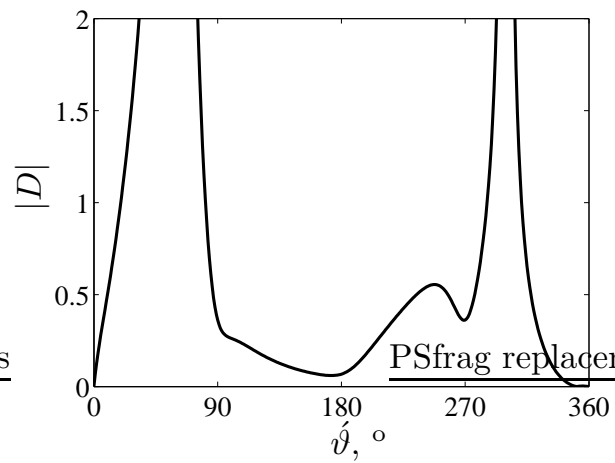

d

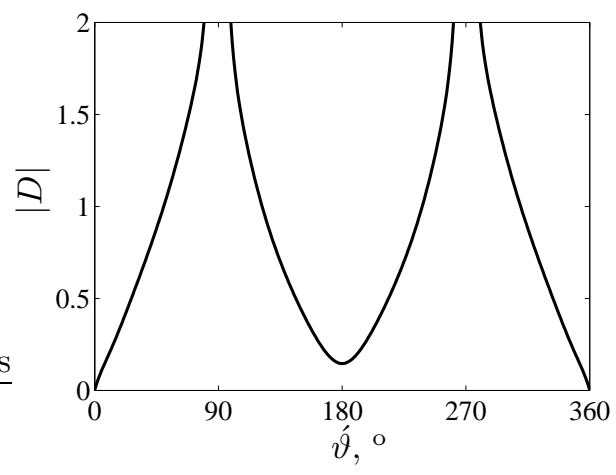

e

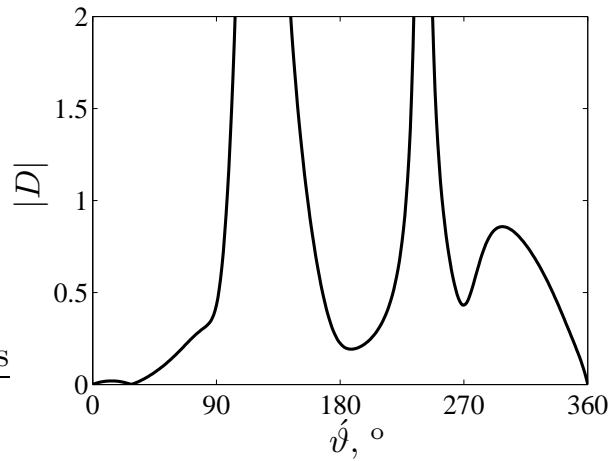

f

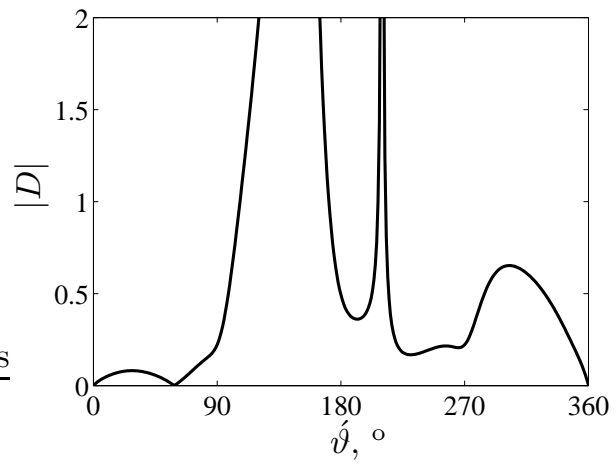

Fig. C.1. Magnitude $D^{(q P, q P)}$ versus $\hat{\vartheta}$, $\varphi^{i n c}=90^{0}$ and $\hat{\vartheta}^{i n c}=0^{0}(\mathrm{a}), 30^{\circ}(\mathrm{b}), 60^{\circ}(\mathrm{c}), 90^{0}(\mathrm{~d}), 120^{\circ}(\mathrm{e})$, $150^{\circ}(\mathrm{f})$. 

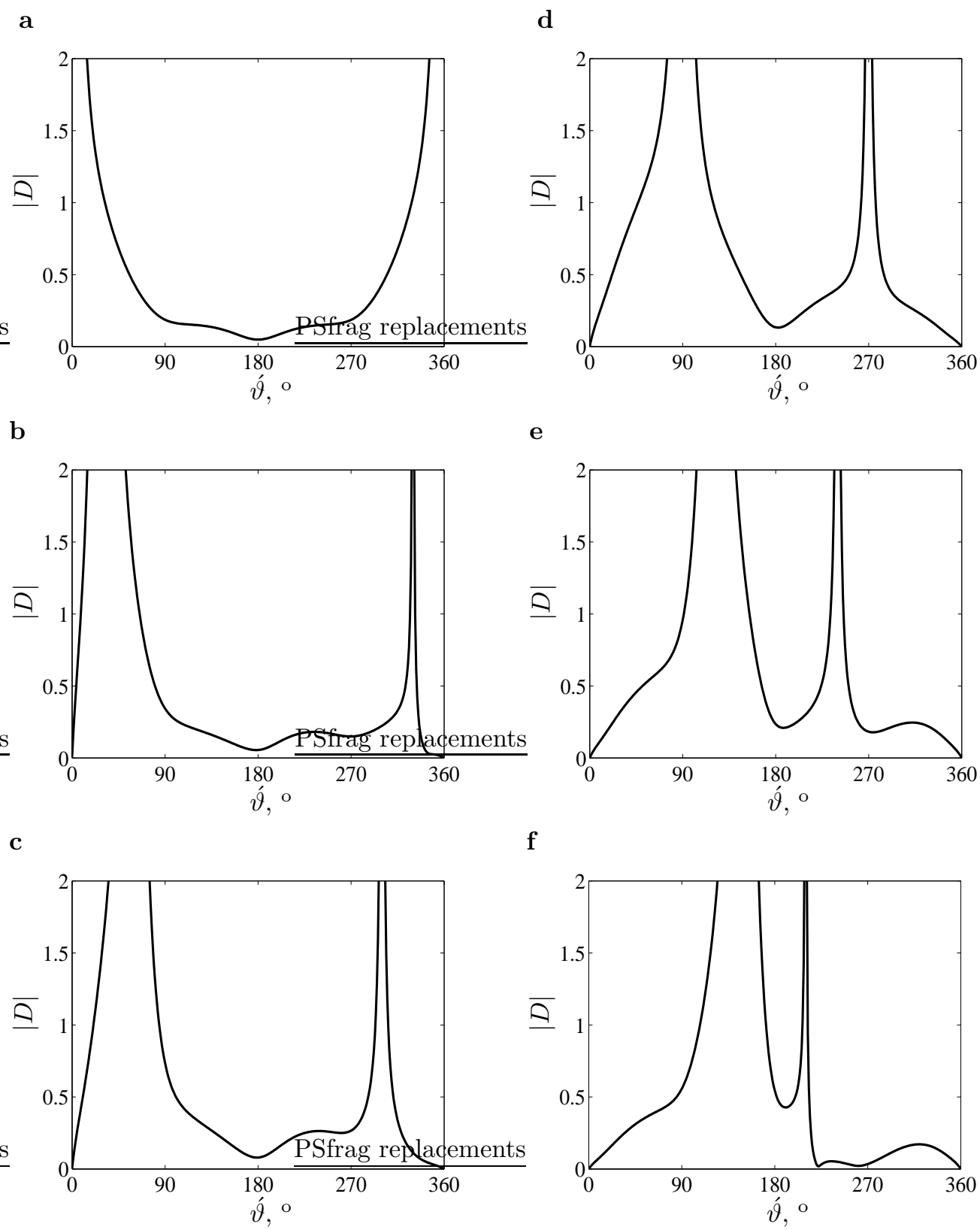

Fig. C.2. Magnitude $D^{(q P, q P)}$ versus $\dot{\vartheta}, \varphi^{i n c}=60^{0}$ and $\vartheta^{i n c}=0^{0}(\mathrm{a}), 30^{0}(\mathrm{~b}), 60^{\circ}(\mathrm{c}), 90^{\circ}(\mathrm{d}), 120^{0}(\mathrm{e})$, $150^{\circ}(\mathrm{f})$. 
a

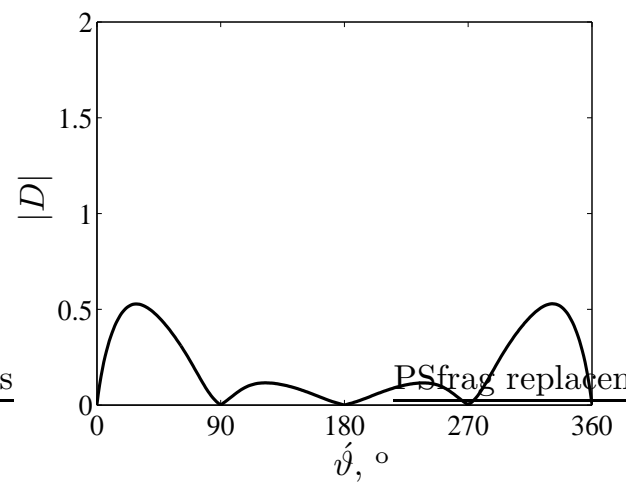

b

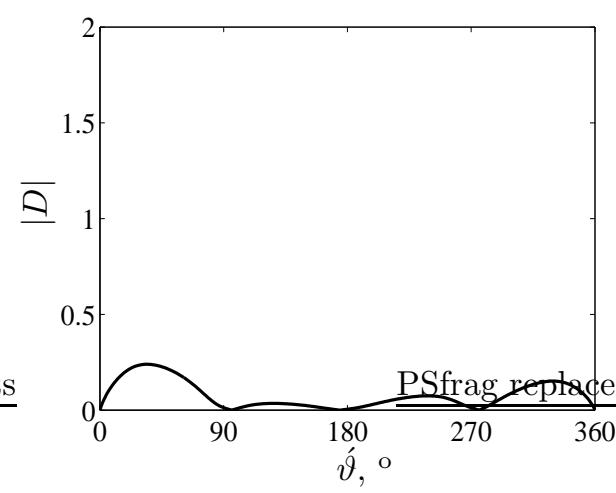

c

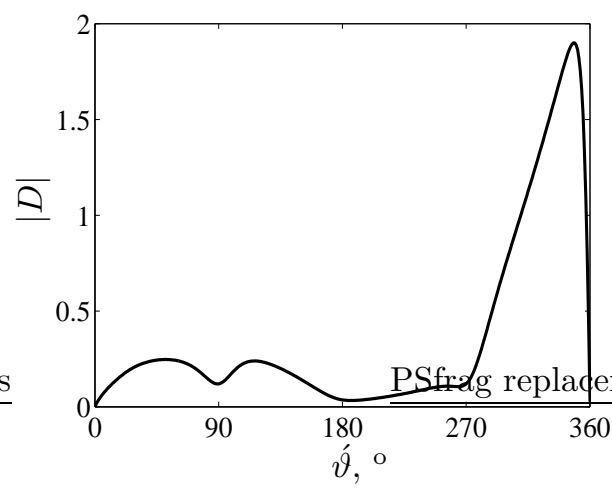

d

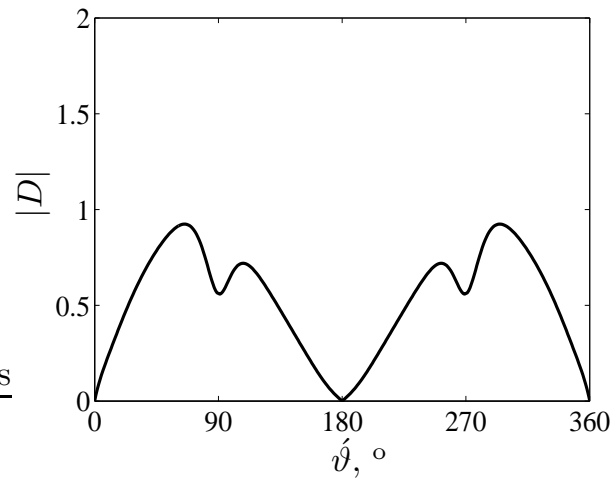

e

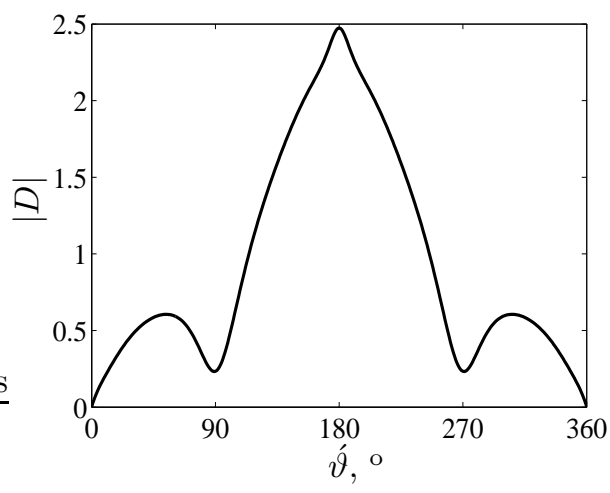

f

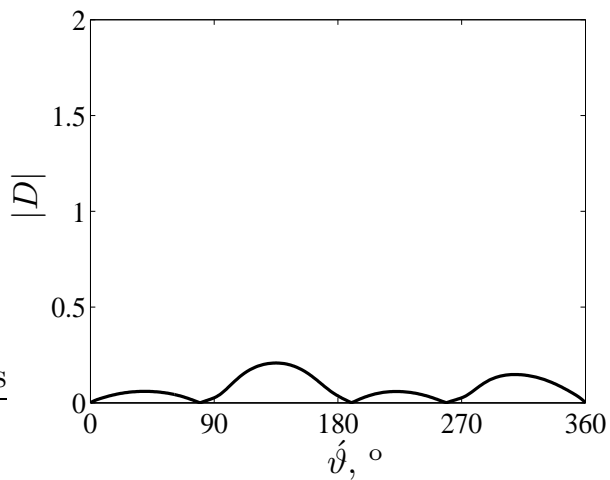

Fig. C.3. Magnitude $D^{(q P, q S V)}$ versus $\dot{\vartheta}, \varphi^{i n c}=90^{\circ}$ and $\hat{\vartheta}^{i n c}=0^{0}(\mathrm{a}), 30^{\circ}(\mathrm{b}), 60^{0}(\mathrm{c}), 90^{\circ}(\mathrm{d}), 120^{0}(\mathrm{e})$, $150^{\circ}(\mathrm{f})$. 
a

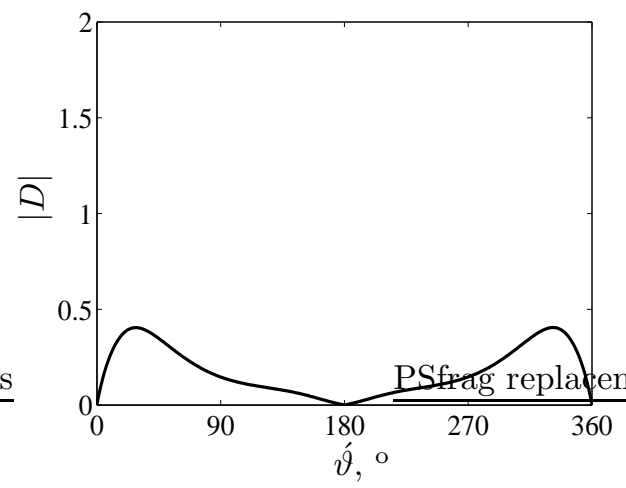

b

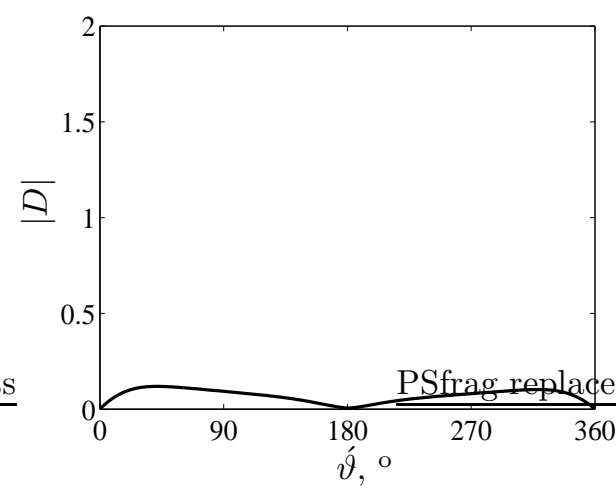

c

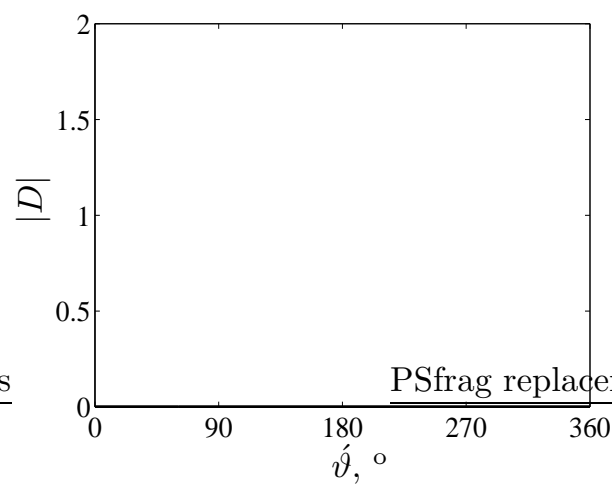

d

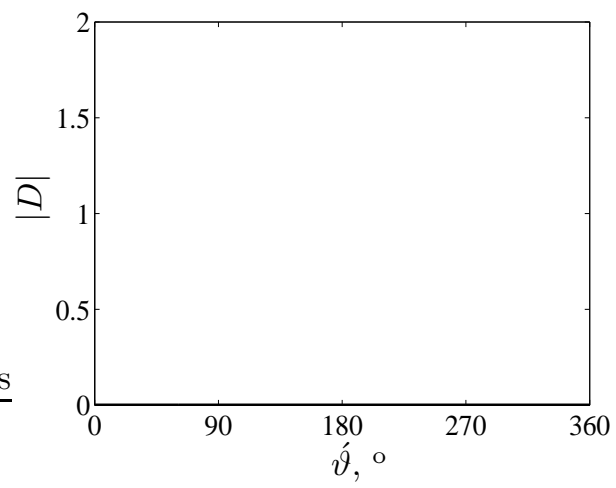

e

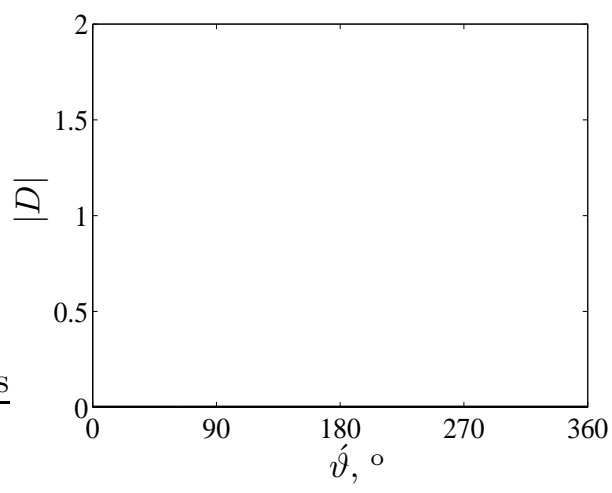

f

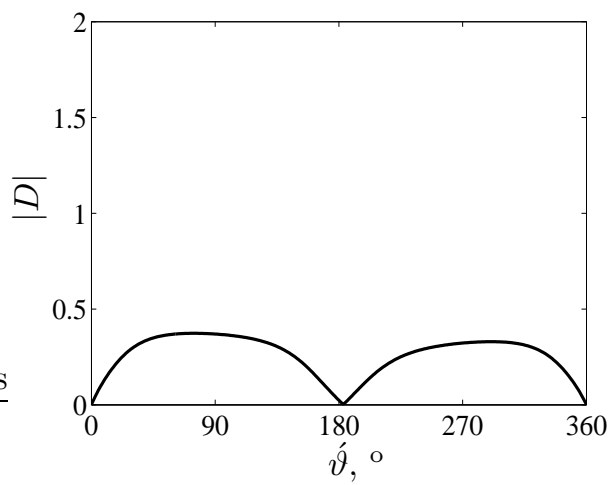

Fig. C.4. Magnitude $D^{(q P, q S V)}$ versus $\dot{\vartheta}, \varphi^{i n c}=60^{0}$ and $\hat{\vartheta}^{i n c}=0^{0}(\mathrm{a}), 30^{\circ}(\mathrm{b}), 60^{0}(\mathrm{c}), 90^{0}(\mathrm{~d}), 120^{0}(\mathrm{e})$, $150^{\circ}(\mathrm{f})$. 
a

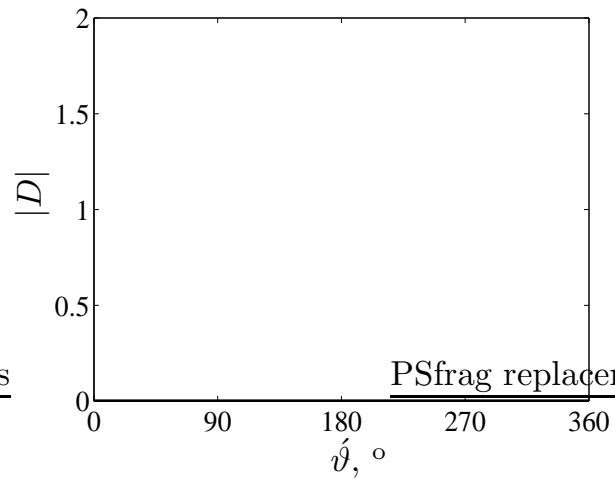

b

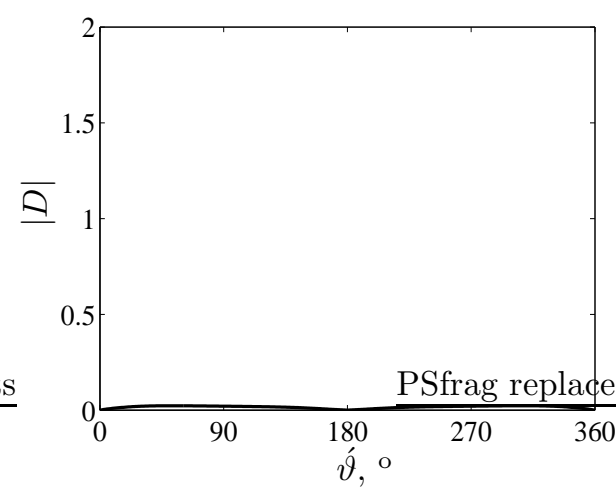

c

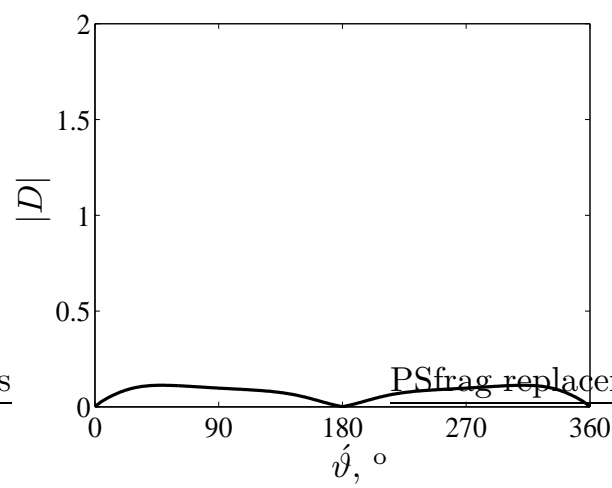

d

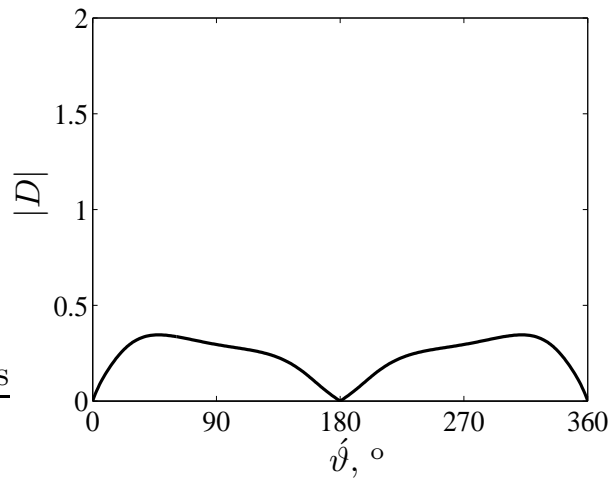

e

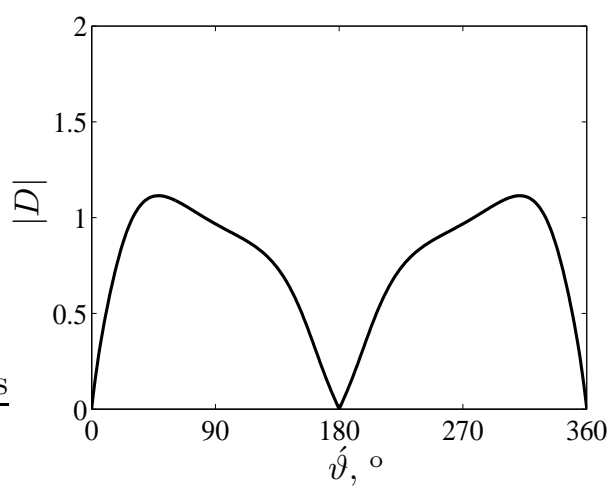

f

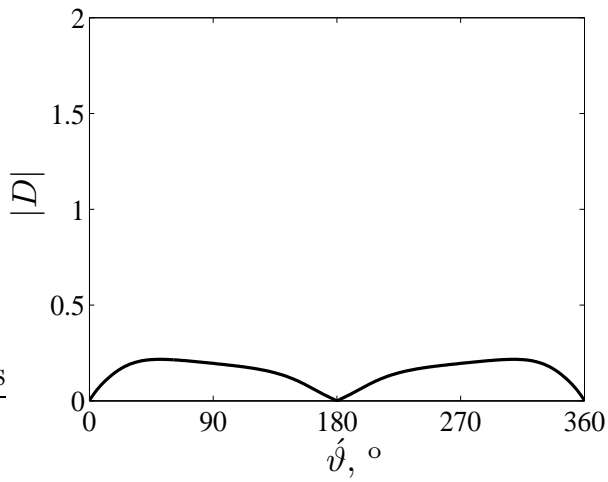

Fig. C.5. Magnitude $D^{(q P, q S H)}$ versus $\dot{\vartheta}, \varphi^{i n c}=60^{0}$ and $\vartheta^{i n c}=0^{0}(\mathrm{a}), 30^{\circ}(\mathrm{b}), 60^{\circ}(\mathrm{c}), 90^{\circ}(\mathrm{d}), 120^{\circ}(\mathrm{e})$, $150^{0}$ (f). 
a

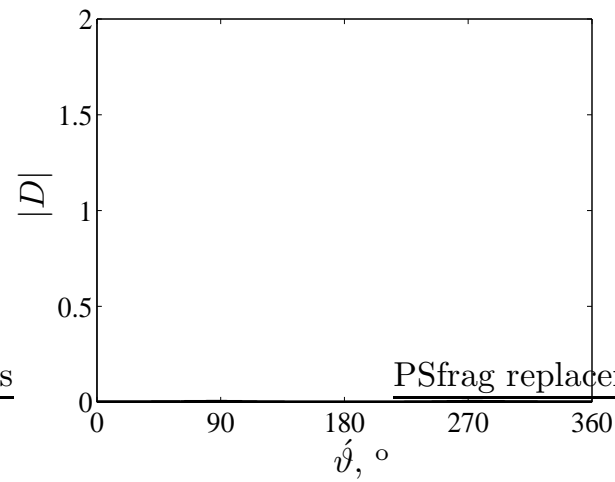

b

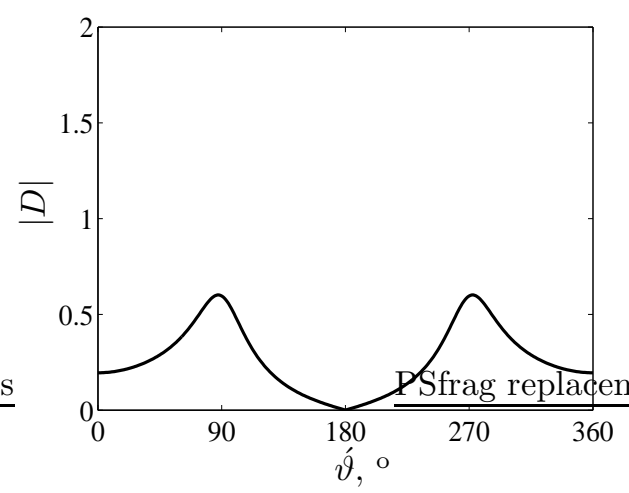

c

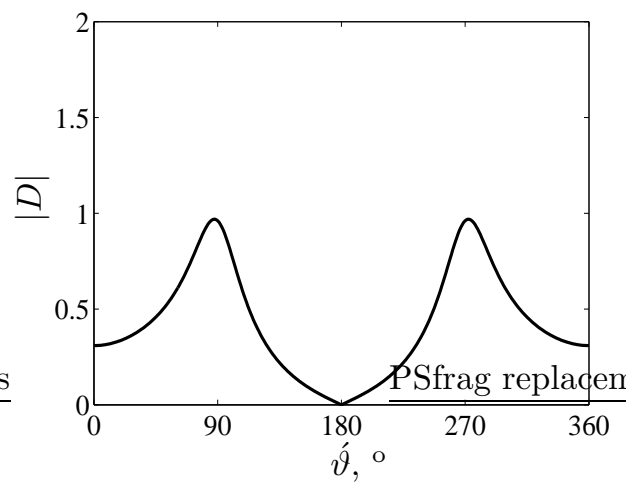

d

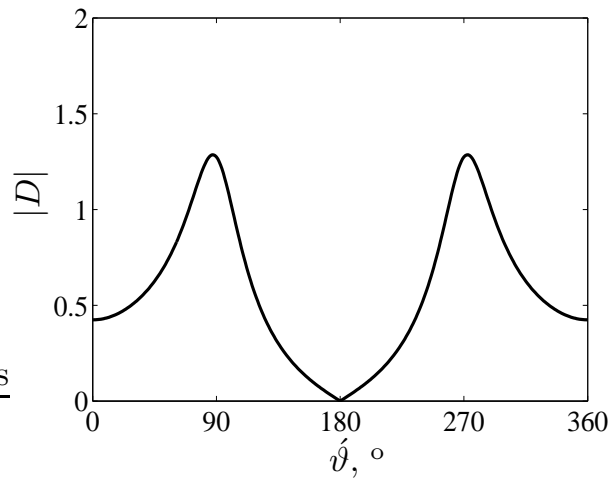

e

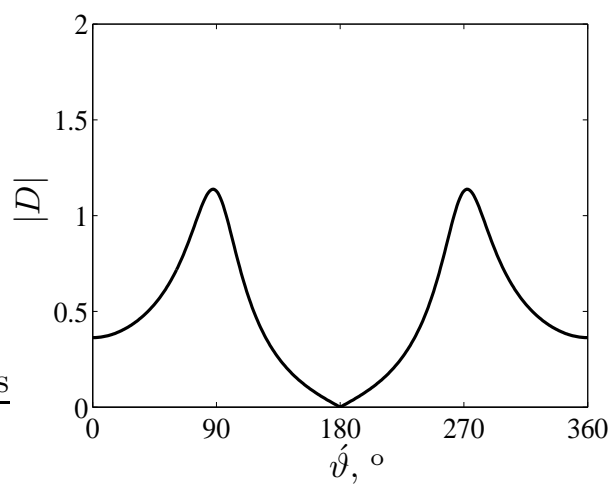

f

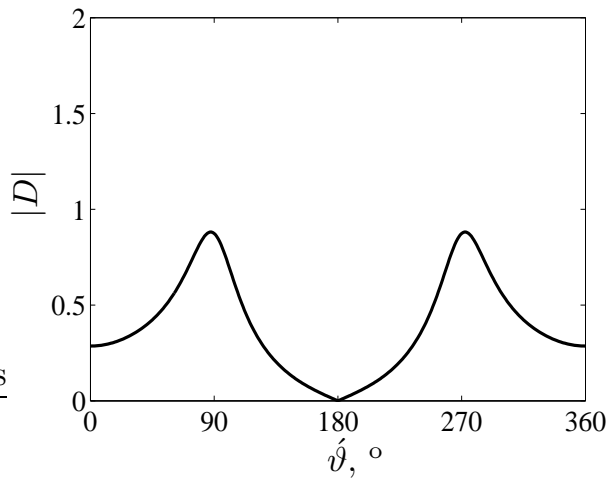

Fig. C.6. Magnitude $D^{(q S H, q P)}$ versus $\dot{\vartheta}, \varphi^{i n c}=60^{0}$ and $\hat{\vartheta}^{i n c}=0^{0}(\mathrm{a}), 30^{0}(\mathrm{~b}), 60^{0}(\mathrm{c}), 90^{0}(\mathrm{~d}), 120^{0}(\mathrm{e})$, $150^{\circ}(\mathrm{f})$. 
a

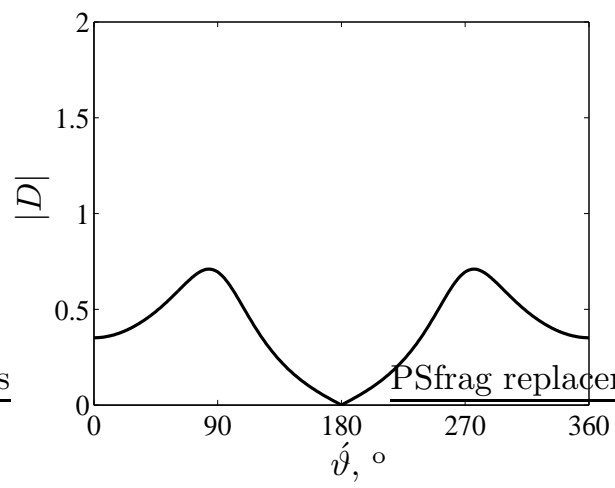

b

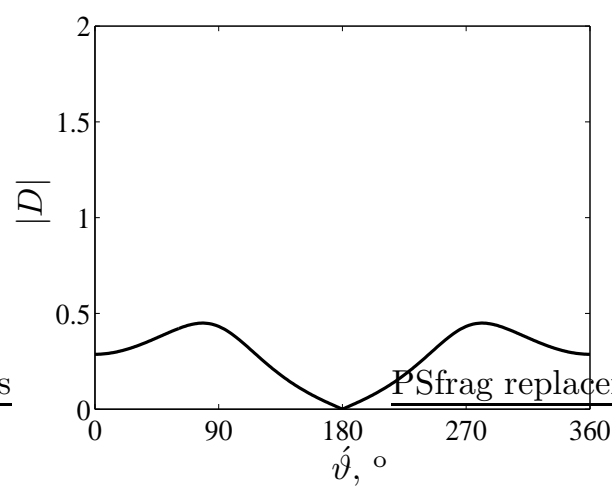

c

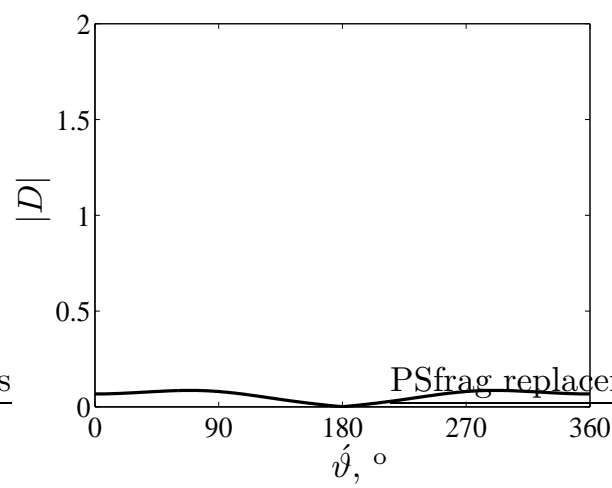

d

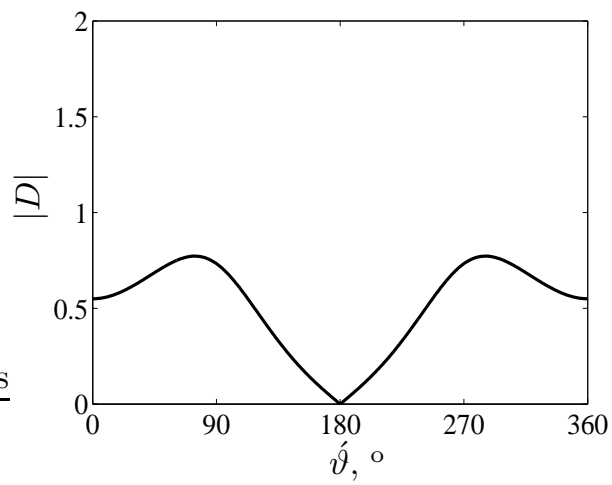

e

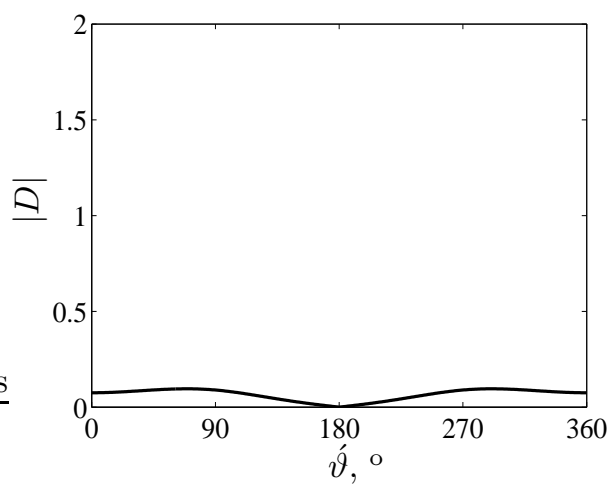

f

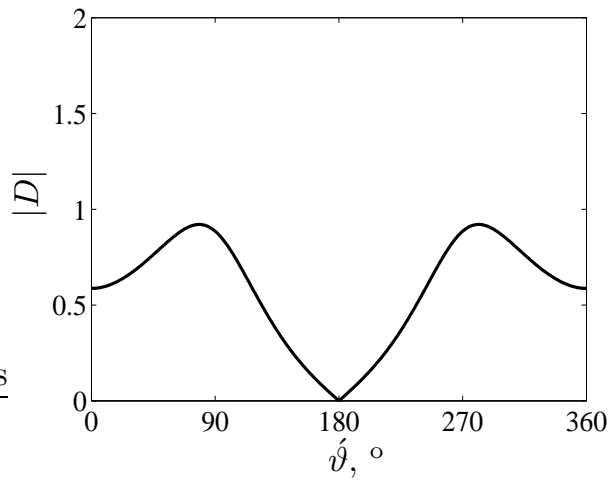

Fig. C.7. Magnitude $D^{(q S H, q S V)}$ versus $\dot{\vartheta}, \varphi^{i n c}=60^{\circ}$ and $\vartheta^{i n c}=0^{0}(\mathrm{a}), 30^{0}(\mathrm{~b}), 60^{0}(\mathrm{c}), 90^{\circ}(\mathrm{d}), 120^{\circ}(\mathrm{e})$, $150^{0}$ (f). 

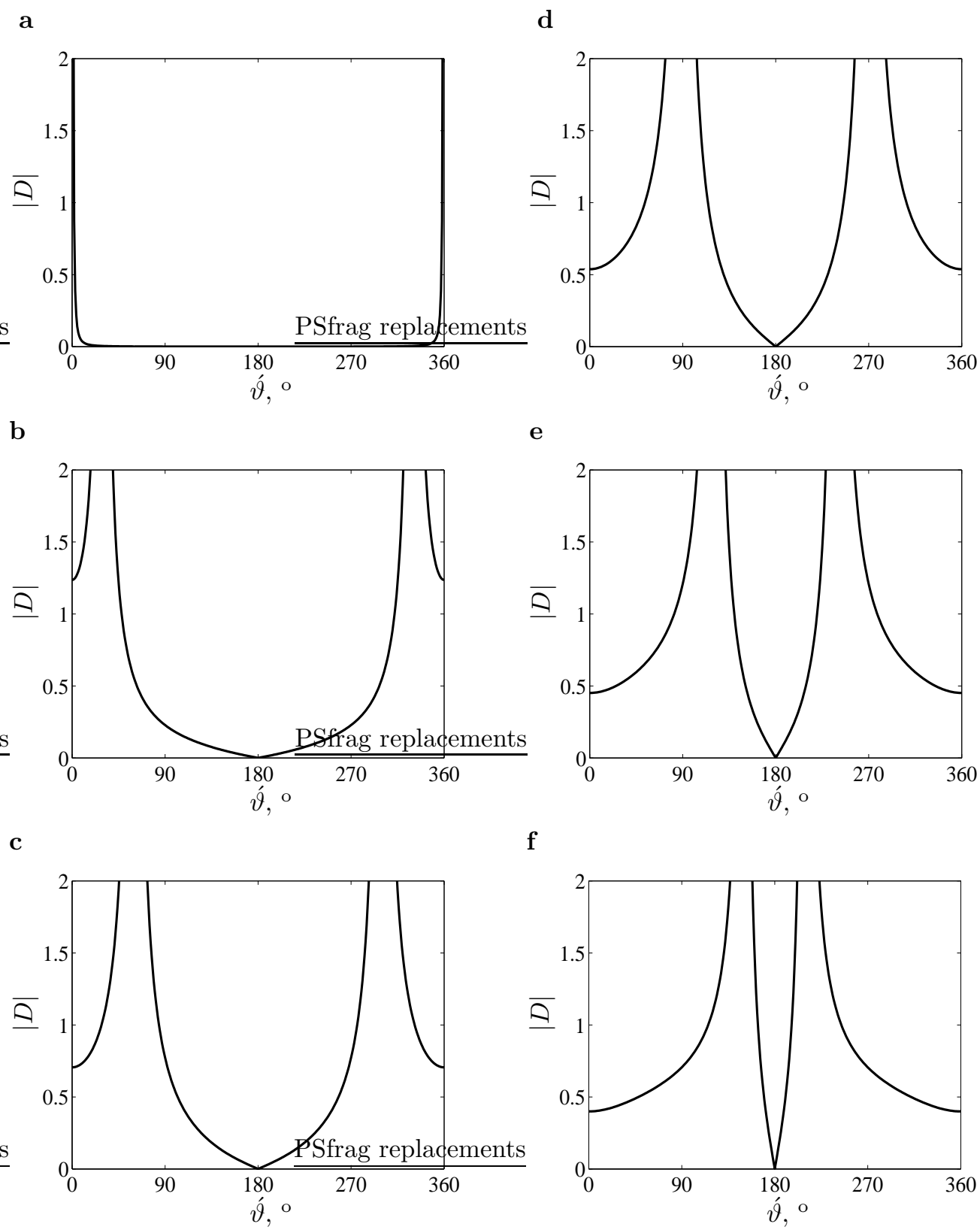

Fig. C.8. Magnitude $D^{(q S H, q S H)}$ versus $\dot{\vartheta}, \varphi^{i n c}=90^{0}$ and $\dot{\vartheta}^{i n c}=0^{0}(\mathrm{a}), 30^{\circ}(\mathrm{b}), 60^{0}(\mathrm{c}), 90^{0}(\mathrm{~d}), 120^{0}(\mathrm{e})$, $150^{0}$ (f). 

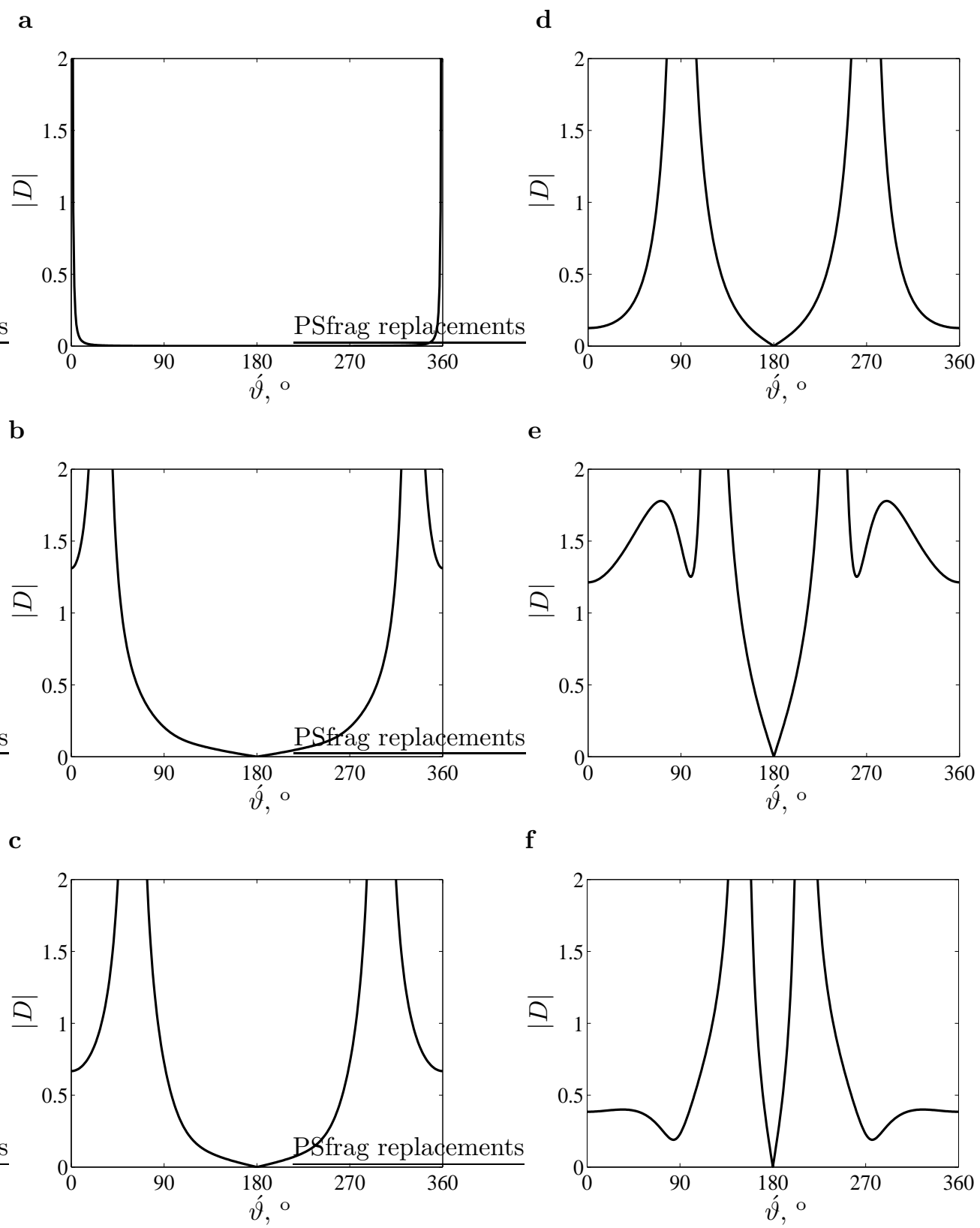

Fig. C.9. Magnitude $D^{(q S H, q S H)}$ versus $\dot{\vartheta}, \varphi^{i n c}=60^{0}$ and $\dot{\vartheta}^{i n c}=0^{0}(\mathrm{a}), 30^{\circ}(\mathrm{b}), 60^{0}(\mathrm{c}), 90^{0}(\mathrm{~d}), 120^{0}(\mathrm{e})$, $150^{0}$ (f). 
Appendix D. Magnitudes of various diffraction coefficients for the axis of symmetry lying in the crack plane perpendicularly to the crack edge

a

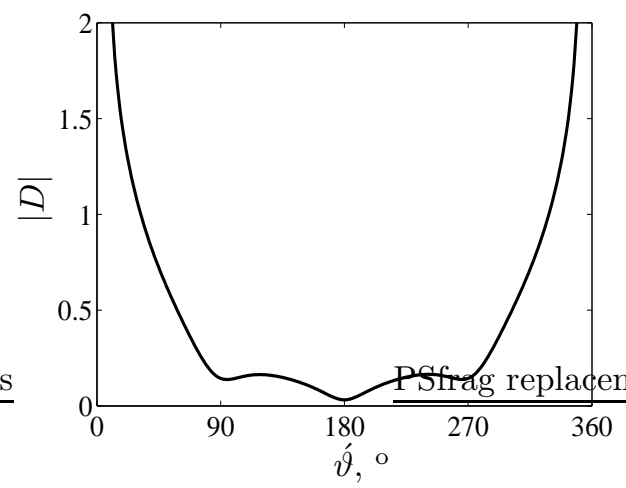

b

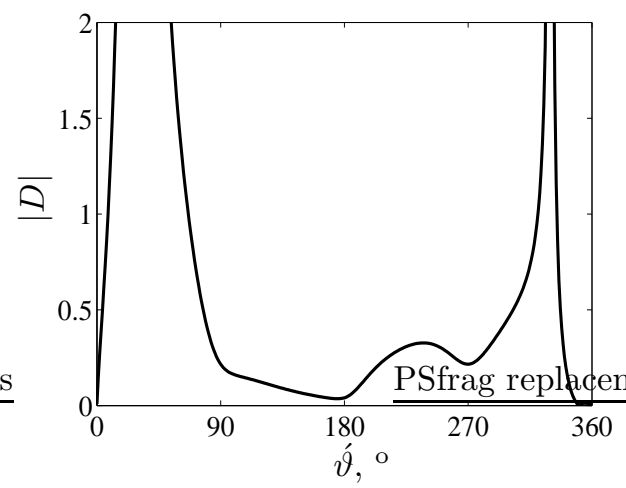

c

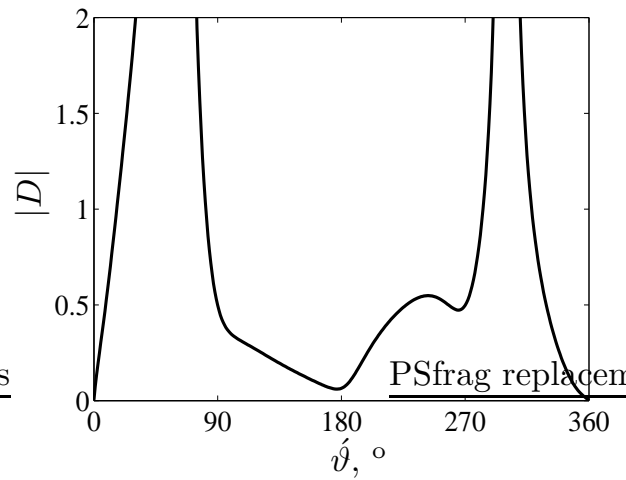

d

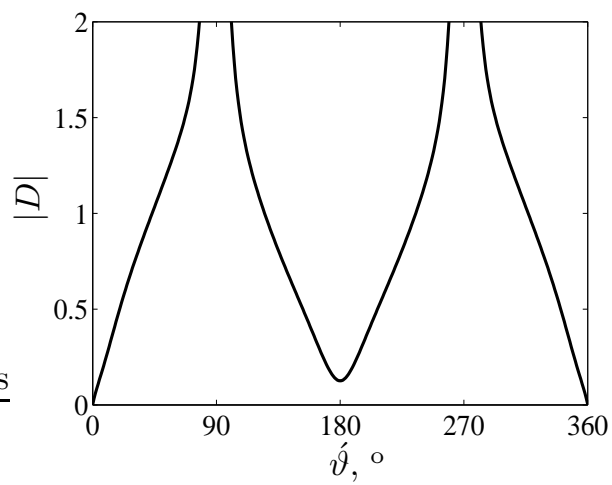

e

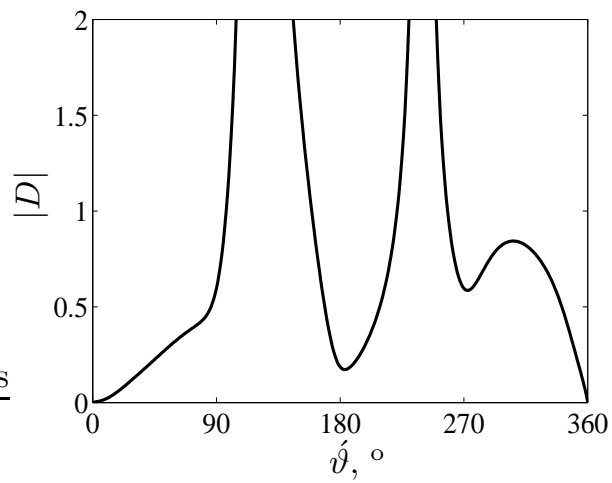

f

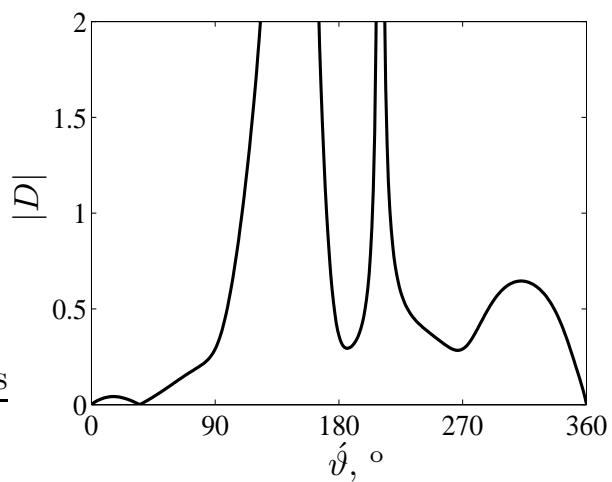

Fig. D.1. Magnitude $D^{(q P, q P)}$ versus $\grave{\vartheta}, \hat{\varphi}^{i n c}=90^{0}$ and $\hat{\vartheta}^{i n c}=0^{0}(\mathrm{a}), 30^{\circ}(\mathrm{b}), 60^{\circ}(\mathrm{c}), 90^{\circ}(\mathrm{d}), 120^{\circ}(\mathrm{e})$, $150^{\circ}(\mathrm{f})$. 
a

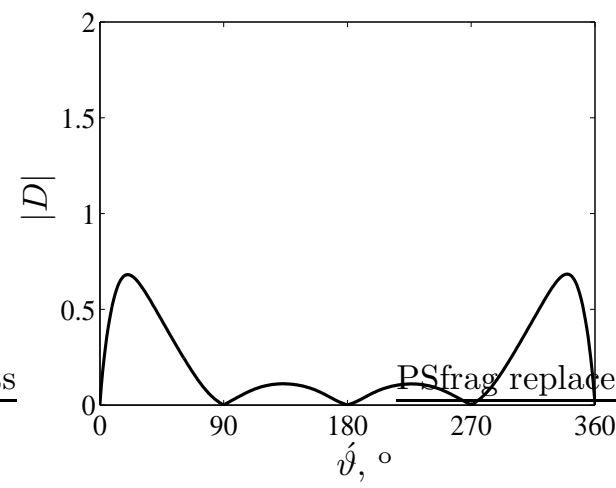

b

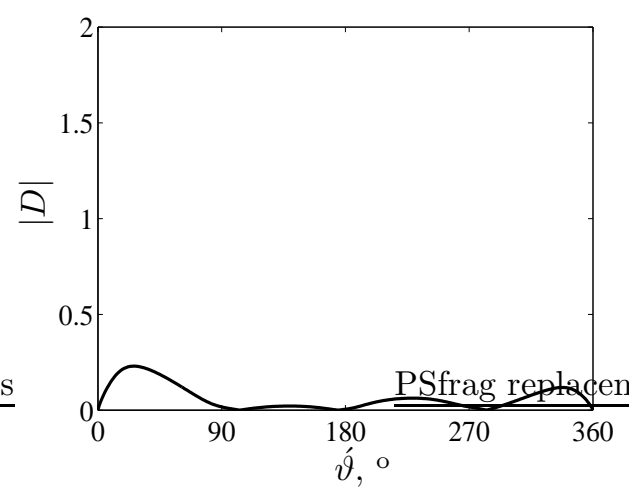

c

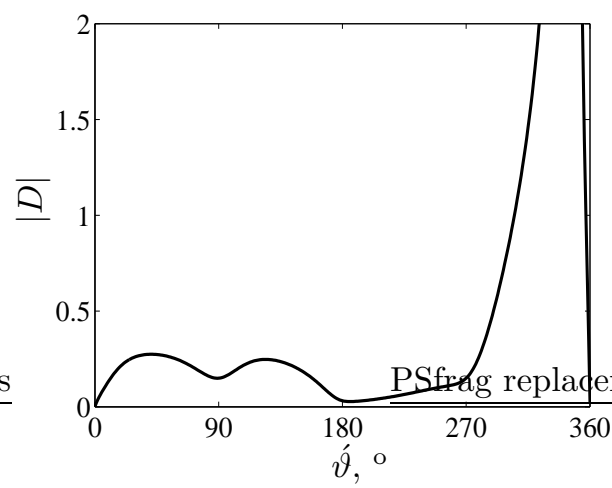

d

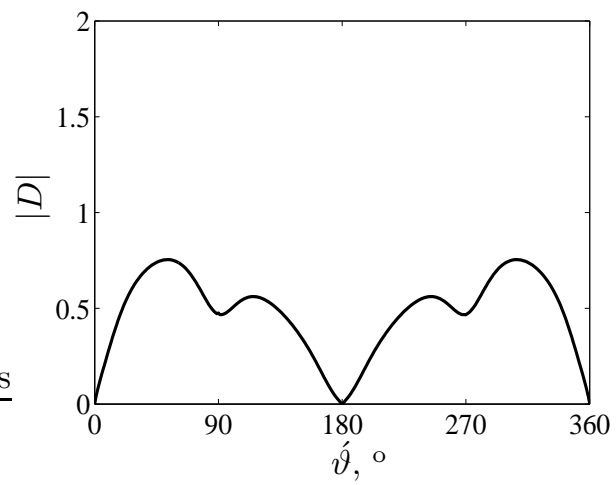

e

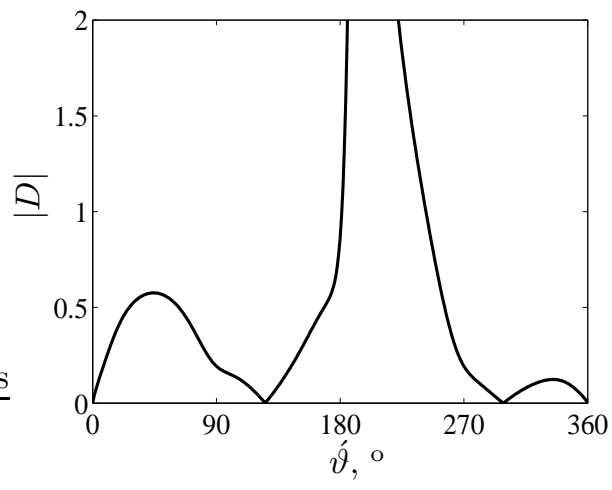

f

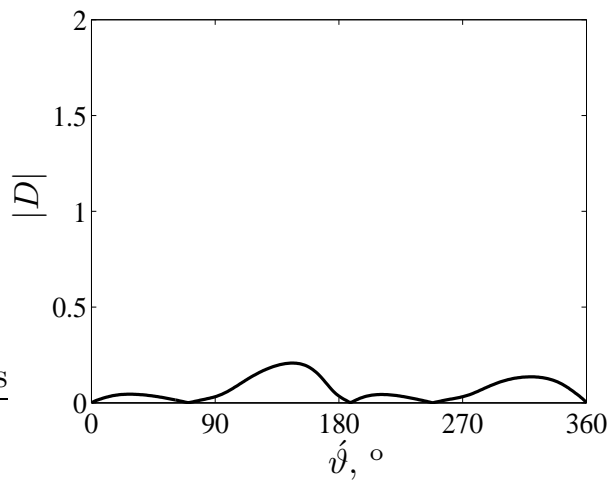

Fig. D.2. Magnitude $D^{(q P, q S V)}$ versus $\dot{\vartheta}, \varphi^{i n c}=90^{\circ}$ and $\dot{\vartheta}^{i n c}=0^{0}(\mathrm{a}), 30^{\circ}(\mathrm{b}), 60^{0}(\mathrm{c}), 90^{\circ}(\mathrm{d}), 120^{0}(\mathrm{e})$, $150^{\circ}(\mathrm{f})$. 
a

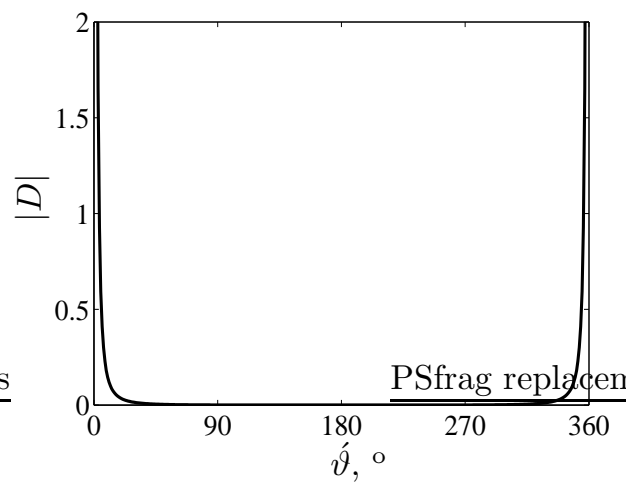

b

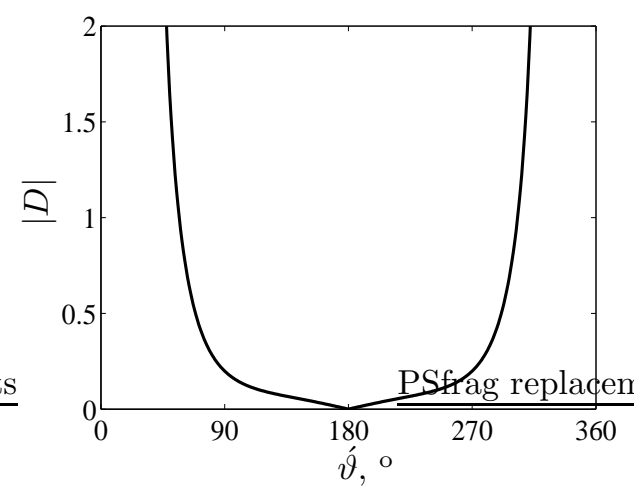

c

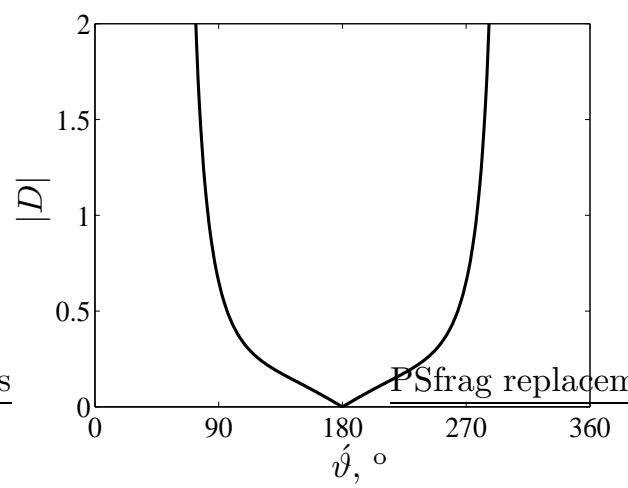

d

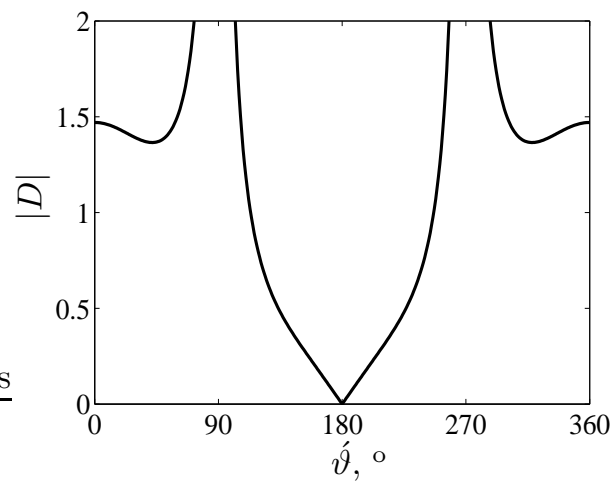

e

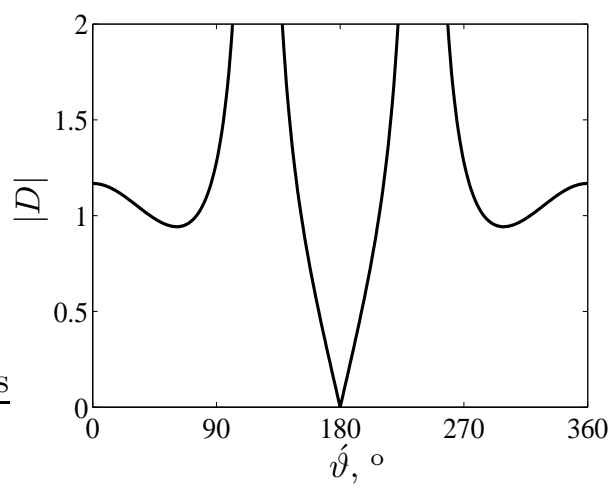

f

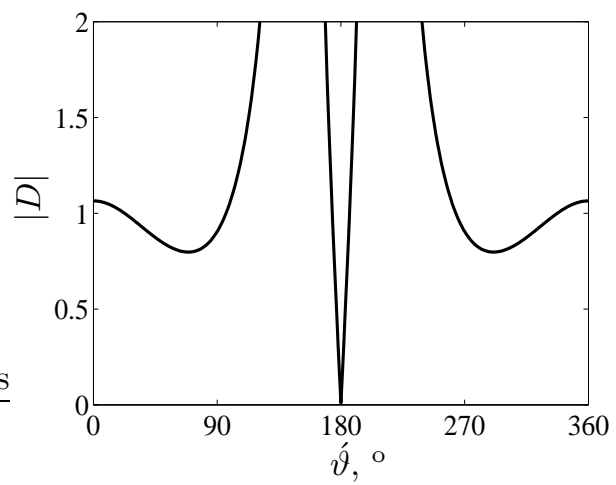

Fig. D.3. Magnitude $D^{(q S H, q S H)}$ versus $\hat{\vartheta}, \varphi^{i n c}=90^{0}$ and $\vartheta^{i n c}=0^{0}(\mathrm{a}), 30^{\circ}(\mathrm{b}), 60^{0}(\mathrm{c}), 90^{\circ}(\mathrm{d}), 120^{0}(\mathrm{e})$, $150^{0}$ (f). 


\section{Appendix E. The effect of cuspidal edges and conical points}

As mentioned at the end of Section 8, the cuspidal edges and conical points on the $q S V$ wave surfaces lead to extra singularities in (123). In Fig. E.1 we plot the "diffraction coefficients" as defined by (123) for the case of the symmetry axis perpendicular to the crack.

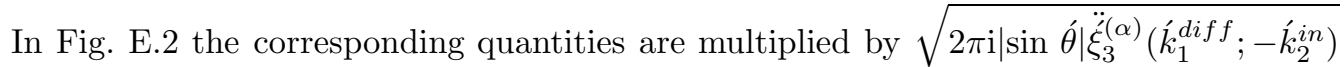
to exclude the influence of the cuspidal edges. In Fig. E.3 further multiplication by $\lambda_{\dot{\xi}_{3}}^{(\alpha)}\left(\dot{\mathbf{k}}^{d i f f}\right)$ is carried out to exclude the influence of the conical points too. The remaining singularities are due to shadow boundaries.

a

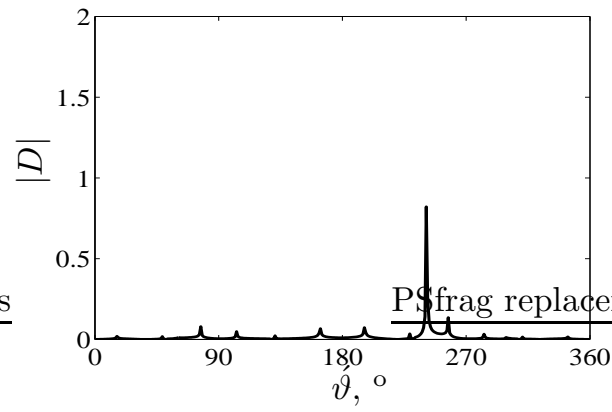

b

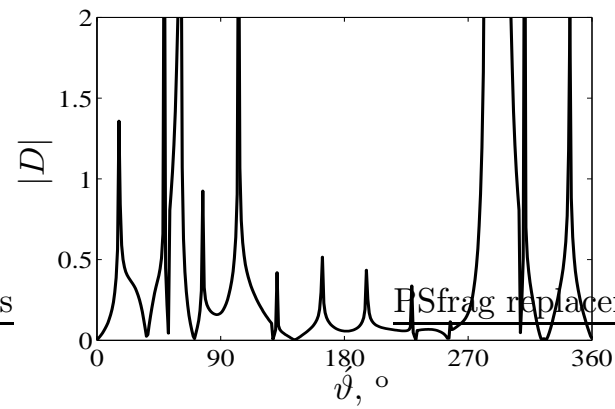

c

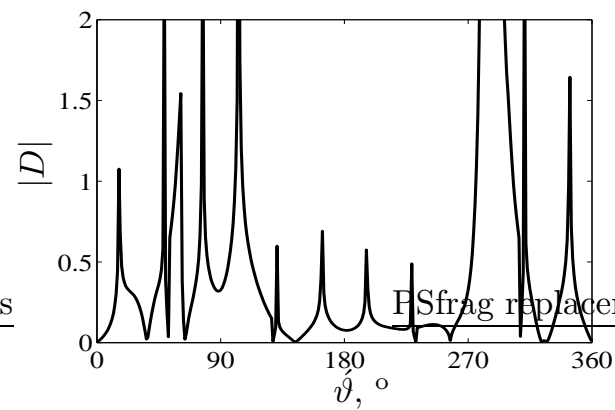

d

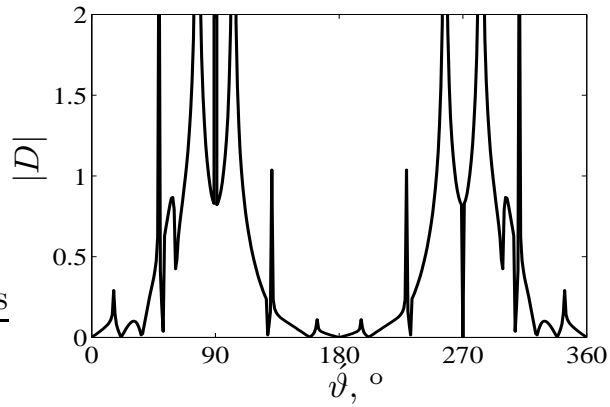

e

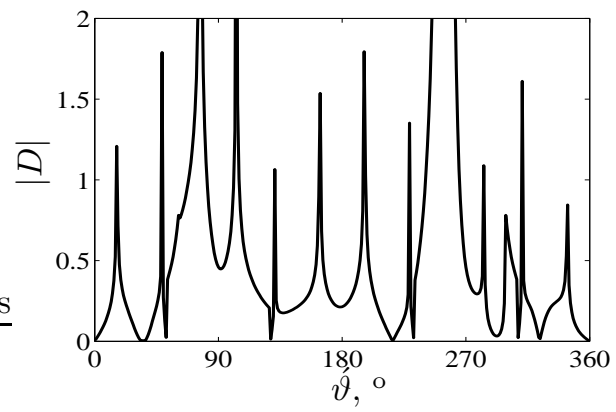

f

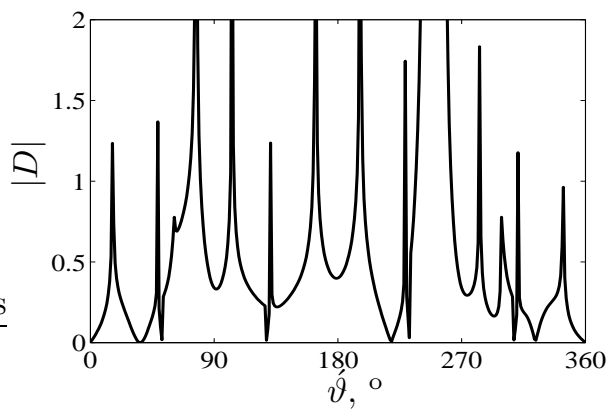

Fig. E.1. Magnitude $D^{(q S V, q P)}$ versus $\dot{\vartheta}, \varphi^{i n c}=90^{0}$ and $\dot{\vartheta}^{i n c}=0^{0}(\mathrm{a}), 30^{0}(\mathrm{~b}), 60^{0}(\mathrm{c}), 90^{0}(\mathrm{~d}), 120^{0}(\mathrm{e})$, $150^{0}(\mathrm{f})$. 

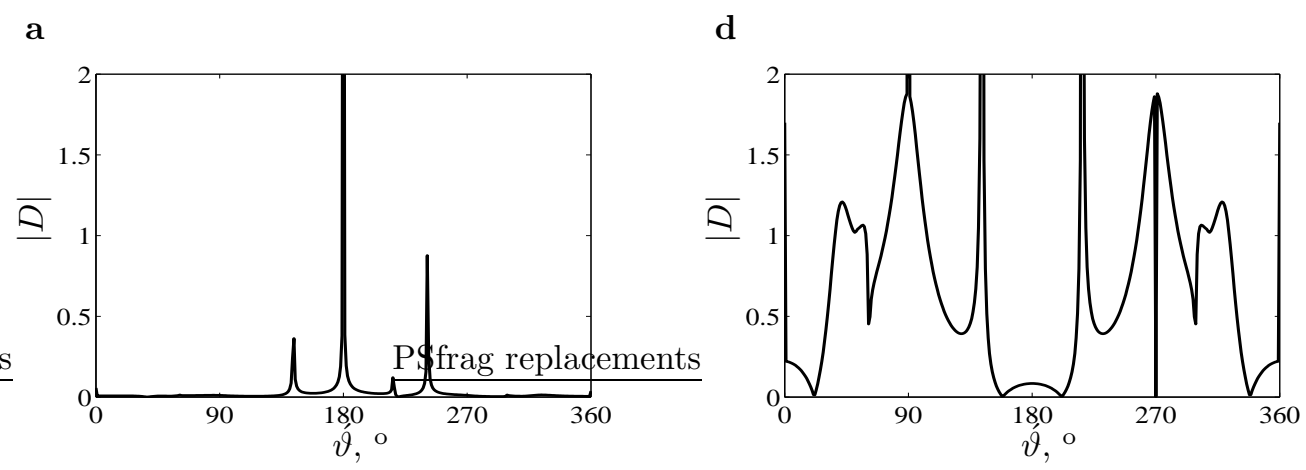

b

e
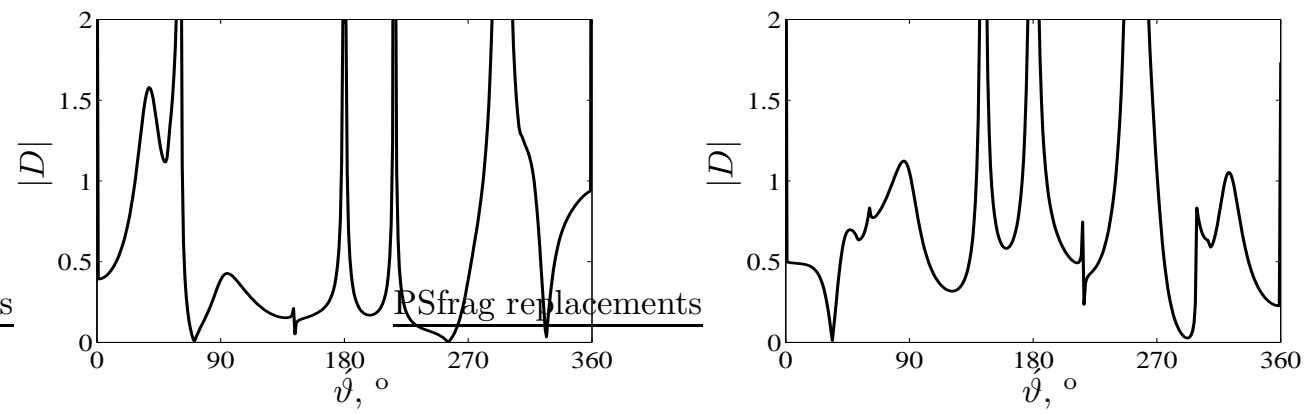

c

f
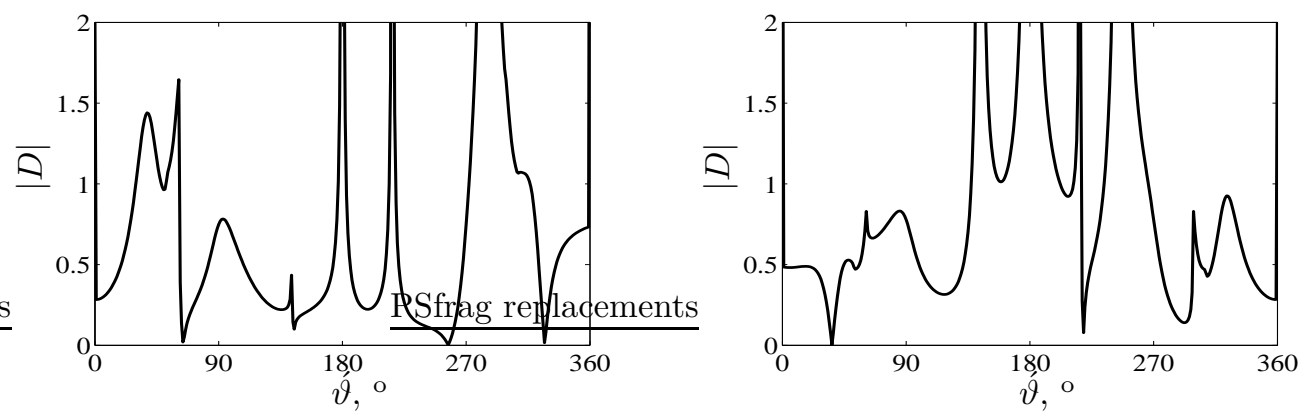

Fig. E.2. As in Fig. E.1 but with the effect of cuspidal edges excluded. 

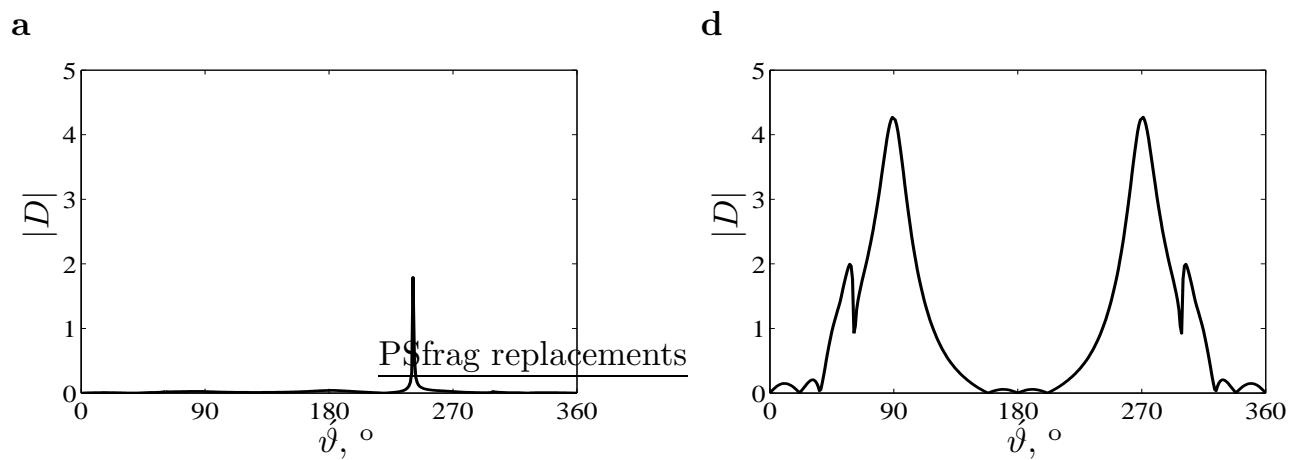

b

e
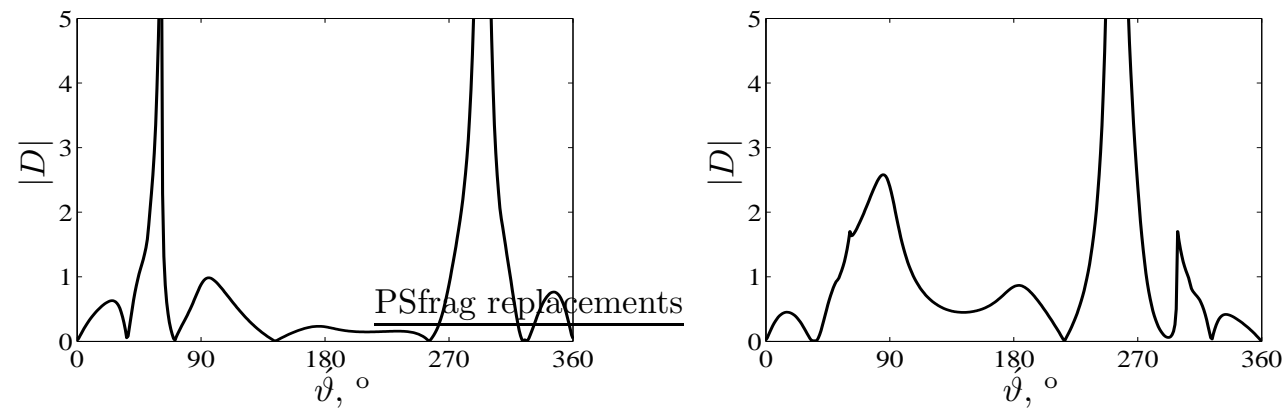

c

f
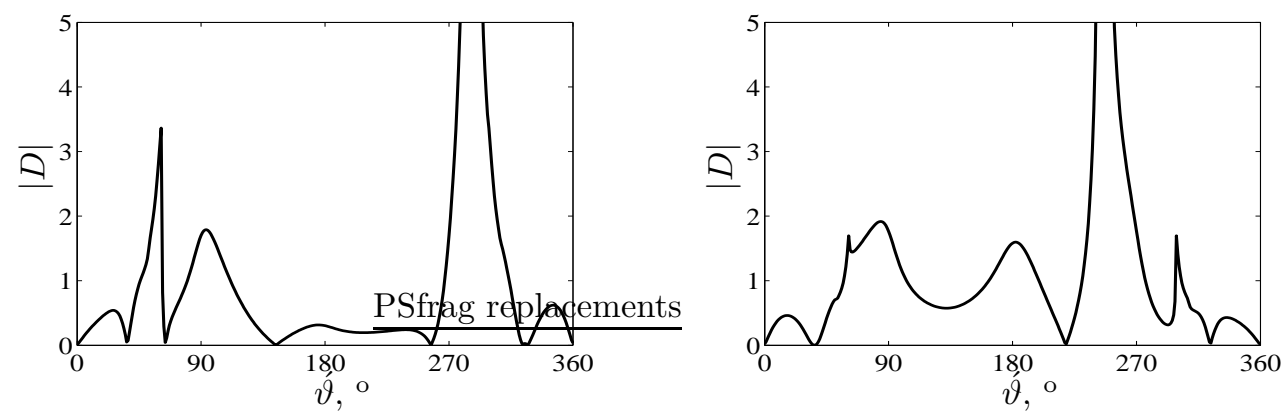

Fig. E.3. As in Fig. E.2 but with the effect of conical points excluded. 